\title{
BUILDING VERTEX ALGEBRAS FROM PARTS
}

\author{
SCOTT CARNAHAN
}

\begin{abstract}
Given a collection of modules of a vertex algebra parametrized by an abelian group, together with one dimensional spaces of composable intertwining operators, we assign a canonical element of the cohomology of an Eilenberg-Mac Lane space. This element describes the obstruction to locality, as the vanishing of this element is equivalent to the existence of a vertex algebra structure with multiplication given by our intertwining operators, and given existence, the structure is unique up to isomorphism. The homological obstruction reduces to an "evenness" problem that naturally vanishes for 2-divisible groups, so simple currents organized into odd order abelian groups always produce vertex algebras. Furthermore, in cases most relevant to conformal field theory (i.e., when we have well-behaved contragradients and tensor products), we obtain our spaces of intertwining operators naturally, and the evenness obstruction reduces to the question of whether the contragradient bilinear form on certain order 2 currents is symmetric or skew-symmetric. We show that if we are given a simple regular VOA and integral-weight modules parametrized by a group of even units in the fusion ring, then the direct sum admits the structure of a simple regular VOA, called the simple current extension, and this structure is unique up to isomorphism.
\end{abstract}

\section{Contents}

1. Notation and definitions 4

1.1. Formal power series 4

1.2. Cohomology of a two-fold delooping $\quad 10$

1.3. Structured vertex algebras 11

1.4. Modules and integral-weight intertwining operators 13

2. Obstruction theory

2.1. Associativity 17

2.2. Commutativity

2.3. Evenness 24

2.4. Modules and intertwining operators 26

3. Quasi-simple current extensions $\quad 29$

3.1. Restricted quasi-simple currents $\quad 29$

3.2. Contragradients and regularity

\begin{tabular}{ll} 
References & 36 \\
\hline
\end{tabular}

0.0.1. Introduction. In this paper, we construct vertex algebras from smaller pieces, by defining a multiplication operation that connects the pieces. The question of extending vertex algebras is about as old as the theory of vertex algebras itself, although the language of modules and intertwining operators took some time to be developed. Lattice vertex algebras are formed from modules for the Heisenberg subalgebra [Borcherds 1986], and the Monster vertex operator algebra, constructed in Frenkel-Lepowsky-Meurman 1988, was later interpreted in Feingold-Frenkel-Ries 1991 and Huang 1994 as a sum of a vertex algebra and a module, with a multiplication operation defined using intertwining operators. More recent work includes Höhn's reformulation of the extension 
problem in terms of three dimensional topological field theory [Höhn 2002], and Huang-KirillovLepowsky's correspondence between extensions of a regular VOA $V$ and commutative associative algebras in the vertex tensor category of $V$-modules [Huang-Kirillov-Lepowsky 2014].

Our goal is to prove existence statements in situations where we have very little information about the fine structure of modules and intertwining operators. We find sufficient conditions for the compatibility of intertwining operators under composition, such that the sum of modules is a vertex algebra. From there, we seek ways to guarantee that these conditions are satisfied in as automatic a fashion as possible. In particular, the last part of the paper is concerned with assembly from a minor generalization of simple currents, which are modules whose intertwining operators are "as nice as possible".

Simple current extensions were described for vertex operator algebras in Dong-Li-Mason 1995, following their introduction in the physics literature in [Schellekens-Yankielowicz 1989]. Similar ideas were articulated earlier in Fuchs-Gepner 1988 and Gepner-Witten 1986. The conditions introduced in [Schellekens-Yankielowicz 1989] section 2 are the following: currents have simple monodromy (i.e., the intertwining operators are integral-weight and all OPEs expand in integral powers of $(z-w))$, and all currents have unique fusion rules with all other primary fields. Translating this to vertex algebra language, we say that a $V$-module $M$ is a simple current if for any irreducible $N$, there exists a unique irreducible $X$ such that the spaces of intertwining operators are described by $I_{V}\left(\begin{array}{c}Y \\ M, N\end{array}\right)=\operatorname{Hom}_{V}(X, Y)$ for all $V$-modules $Y$.

There is substantial previous work in this area, with some limited existence results from twisting by weight one semi-primary elements via the operator $\Delta(z)$ (e.g., Dong-Li-Mason 1995 and [Li 1997]), and much more involving explicit constructions from codes via tensor products of the Ising model (starting with Miyamoto 1996a and Dong-Griess-Höhn 1998). In these cases, the intertwining operators are explicitly known, so the existence question amounts to verifying the Jacobi identity. More work has been done concerning the properties of such extensions: Representation theory via induced modules (under two different definitions of induced module) is studied in Miyamoto 1996b, Dong-Lin 1994, Lam 2001, and [Yamauchi 2002, and automorphism groups of simple current extensions are examined in Shimakura 2006 and Griess-Lam 2011.

Work on existence has been hampered by the fact that the simplest case, given by an order 2 extension, contains essentially all of the subtle complexity of the general case. Indeed, our methods of reduction solve all problems except those that we see in the order 2 case. More specifically, in this paper, we show that simple currents parametrized by an abelian group $A$ yield a vertex algebra as long as an evenness obstruction vanishes, and we reduce this obstruction to the triviality of a quadratic form on the elementary abelian 2-group $A / 2 A$. For regular vertex operator algebras, Proposition 2.6 in Lam-Yamauchi 2006] reduces the existence problem in this case to the hypothesis that the canonical contragradient form on an order 2 simple current is symmetric. While we have been unable to show that this assumption holds in general, it does hold in many cases, so we are led to the following:

Evenness conjecture: For any simple self-dual integral-weight module $M$ over a simple $C_{2^{-}}$ cofinite Möbius vertex algebra with invariant bilinear form, the contragradient bilinear form on $M$ is symmetric.

Assuming this conjecture, we can conclude that simple current extensions always exist. Removing the hypothesis of integral weight, we conjecture that the balancing isomorphism, given by braiding any simple current against itself, is the same as multiplication by $e^{2 \pi i L(0)}$. This general statement is also far from new, for example, it is asserted without proof for the case of modular tensor categories associated to rational CFTs in Fuchs-Runkel-Schweigert 2004 Remark 2.15. The analogous result in the theory of local conformal nets on $S^{1}$ is known as the conformal spin-statistics theorem (Theorem 3.13 of Guido-Longo 1995), and it was used to produce a simple current extension result (Lemma 2.1 of Kawahigashi-Longo 2004). Unfortunately, a translation of Guido-Longo's argument to vertex algebra language does not seem to be straightforward. 
There has been substantial progress on the evenness question, both positive and negative, after the main text of this paper was written, and it seems evenness is a much richer property than I had anticipated. The first result in the positive direction is in van Ekeren-Möller-Scheithauer 2015, where the evenness conjecture is shown to hold for simple rational $C_{2}$-cofinite self-contragradient vertex operator algebras of CFT type whose modules have group-like fusion and positive $L(0)$ spectrum. On the negative side, Thomas Creutzig has informed me that the evenness conjecture as stated is in fact too strong, and that symplectic fermions (as an integer-graded odd simple current extension of the triplet VOA $\mathcal{W}(2))$ give a counterexample even in the case of a $C_{2}$-cofinite vertex operator algebra. However, there is a more general positive result in Creutzig-Kanade-Linshaw 2015, that the conjecture holds for vertex operator algebras that are rational, $C_{2}$-cofinite, and unitary. We would be very interested to see additional results concerning satisfactory conditions for evenness and counterexamples.

The main engine behind this paper is a homological obstruction theory, using spaces of intertwining operators to construct a canonical element of $H^{4}\left(K(A, 2), \mathbb{C}^{\times}\right)$, for $A$ a parametrizing abelian group, and $K(A, 2)$ the second Eilenberg-Mac Lane space (i.e., a 2-fold delooping). The cohomology theory in question is far from new, dating to Mac Lane's 1950 ICM address, and its application to vertex algebra theory is also over 20 years old, starting with its role in classifying types of abelian intertwining algebras in Dong-Lepowsky 1993. We also see an application in Proposition 3.29(ii) of [Fuchs-Runkel-Schweigert 2004], characterizing commutative Schellekens algebras in terms of the vanishing of an element of $H^{4}\left(K(A, 2), \mathbb{C}^{\times}\right)$. However, the inherent complexity of the manipulations of 4-cocycles and power series in multiple variables seems to have limited its uses, so much of our work in this paper is an attempt make such calculations avoidable in the future.

Our application of $H^{4}\left(K(A, 2), \mathbb{C}^{\times}\right)$to the theory of vertex algebras differs from previous work in the following important sense: We obtain abstract existence, without requiring a collection of multiplications to be chosen ahead of time. In contrast, Dong-Li-Mason 1995 uses an explicit choice of intertwining operators, and [Höhn 2002] requires one to start with an abelian intertwining algebra before cutting out a subspace. This difference is why we have to do the messy work of describing associativity and commutativity data, and why the evenness problem appears.

There are certainly plenty of directions to go beyond the content of this paper. For example extensions by modules that aren't simple currents are a topic of active interest, especially among people seeking constructions of holomorphic VOAs of central charge 24 following Schellekens's list of candidates Schellekens 1992. The formalism of this paper allows for a minor incursion into this domain: One has a non-orbifold decomposition of the holomorphic $E_{8,1}$ VOA into a direct sum of the $G_{2,1} F_{4,1}$ VOA and an irreducible module $X$ with fusion rule $X^{2}=X+1$. While $X$ is not a simple current, we may, by setting the intertwining operator $X \otimes X \rightarrow X((z))$ to zero, form a vertex algebra $1+X$ with the same character as $E_{8,1}$, but where $G_{2,1} F_{4,1}$ is the algebra of fixed points of an involution. Also, one may wish to go beyond the integral weight constraint, and construct generalized vertex algebras, where locality comes with a phase defect. Indeed, our original motivation for this work was in generalized moonshine, where we sought to build Lie algebras by assembling twisted modules of $V^{\natural}$ into abelian intertwining algebras. A special case of that construction in the order 2 case was done in Huang 1994 (modulo the sketchy proof of Theorem 3.8), and also in [Höhn 2003] for a different involution. The assembly problem has been fully solved in van Ekeren-Möller-Scheithauer 2015, and we used it in Carnahan 2012 to prove Norton's Generalized Moonshine conjecture. This paper was split off of an earlier version of that paper (which was both longer and conditional on some unavailable results) when I was informed that the integral weight case held independent interest.

0.0.2. Main results. Our first result gives a general sufficient criterion for existence of a vertex algebra structure. It necessarily uses some cumbersome terminology, e.g., a commutativity datum is 
a collection of 1-dimensional spaces of intertwining operators that satisfy some niceness conditions, and $\mathcal{T}$ is a type (e.g., weighted, quasiconformal, equipped with $G$-action).

Theorem 2.2.13). Let $V$ be a $\mathcal{T}$-vertex algebra, let $A$ be an abelian group, and let $\left\{M_{i}\right\}_{i \in A}$ be a set of $V$-modules in $\mathcal{T}$, such that $M_{0}=V$. Given a one dimensional $\mathcal{T}$-commutativity datum, and any normalized choice of nonzero elements $\left\{m_{z}^{i, j} \in \mathcal{I}_{i, j}^{i+j}\right\}_{i, j \in A}$, the following hold:

(1) The action of $C_{a b}^{2}\left(A, \mathbb{C}^{\times}\right)$on $\bigoplus \mathcal{I}_{i, j}^{i+j}$ induces a translation action $\left(\left\{\lambda_{i, j}\right\},(F, \Omega)\right) \mapsto(d \lambda$. $(F, \Omega))$ on the group of abelian 3-cocycles. This action is transitive on representatives of any fixed cohomology class in $H_{a b}^{3}\left(A, \mathbb{C}^{\times}\right)$, with stabilizer given by the group of abelian 2-cocycles $\left\{\lambda_{i, j}\right\}$.

(2) The function $A \rightarrow \pm 1$ defined by $i \mapsto \Omega(i, i)$ is a quadratic form invariant under $\lambda$-twist by 2-cochains. In particular, the abelian cohomology class of $(F, \Omega)$ is canonically attached to the commutativity datum.

(3) If $\left\{M_{i}\right\}$ is $\mathcal{T}$-compatible, then the quadratic form $i \mapsto \Omega(i, i)$ is identically one if and only if there exists a normalized 2-cochain $\lambda$ such that $\left\{\lambda_{i, j} m_{z}^{i, j}\right\}$ describe an A-graded $\mathcal{T}$-vertex algebra structure on $\bigoplus_{i \in A} M_{i}$.

(4) The A-graded $\mathcal{T}$-vertex algebra structure on $\bigoplus_{i \in A} M_{i}$ is unique up to isomorphism if it exists.

Our second result requires an evenness condition on simple currents (and in particular the elements of the fusion ring parametrizing them).

Theorem (3.2.12). Let $V$ be a $C_{2}$-cofinite simple Möbius vertex algebra with finite dimensional weight spaces and invariant bilinear form. For any collection of $V$-modules parametrized by a group of even units in the fusion ring of $V$, the direct sum has the structure of a simple current extension, and this structure is unique up to isomorphism. The simple current extension is a $C_{2}$ cofinite Möbius vertex algebra with finite dimensional weight spaces. If $V$ is also a rational vertex operator algebra, then the simple current extension is also rational, i.e., it is a simple regular vertex operator algebra.

Our third result is conditional on the evenness conjecture.

Theorem (3.2.14). Let $V$ be a $C_{2}$-cofinite simple Möbius vertex algebra with finite dimensional weight spaces and invariant bilinear form. Assuming the evenness conjecture, any collection of integral-weight modules parametrized by a group of units in the fusion ring of $V$ admits the structure of a simple current extension on the direct sum. The simple current extension is a simple $\mathrm{C}_{2}$-cofinite Möbius vertex algebra, and it is unique up to isomorphism. If $V$ is also a rational vertex operator algebra, then the simple current extension is also rational, i.e., it is a simple regular vertex operator algebra.

0.0.3. Acknowledgements. The author would like to thank Tomoyuki Arakawa, Bruce Bartlett, Richard Borcherds, Thomas Creutzig, Yoshitake Hashimoto, André Henriques, Gerald Höhn, Satoshi Kondo, Ching-Hung Lam, Jacob Lurie, Chris Schommer-Pries, Hiroki Shimakura, Hiromichi Yamada, and the anonymous referee for helpful conversations and advice.

This research was partly supported by NSF grant DMS-0354321, JSPS Kakenhi Grant-in-Aid for Young Scientists (B) 24740005, the Program to Disseminate Tenure Tracking System, MEXT, Japan, and the World Premier International Research Center Initiative (WPI Initiative), MEXT, Japan.

\section{Notation AND DEFinitions}

1.1. Formal power series. We will describe basic aspects of power series.

Concretely, we will encounter situations similar to the following: we are given maps from a vector space $W$ to the formal power series vector spaces $V((z))((w))$ and $V((w))((z))$, and we would like 
to say that both maps factor through a common subspace $V[[z, w]]\left[(z-w)^{-1}\right]$. To make sense of such a claim, we need to make an unambiguous choice of inclusions from the latter vector space to the former two. Since this paper is only concerned with power series that have integer exponents, there isn't much ambiguity in the first place.

The methods of formal calculus (see e.g., Frenkel-Lepowsky-Meurman 1988 Chapter 8) provide one way to make such a selection: To expand in the first space, we write $(z-w)^{n}$ as $z^{n}(1-w / z)^{n}$, and use the binomial theorem to get the power series $z^{n} \sum_{k>0}\left(\begin{array}{l}n \\ k\end{array}\right) w^{k} / z^{k}$. To expand in the second space, we identify $(z-w)^{n}$ with $(-(w-z))^{n}$, expand as $(-1)^{n} w^{n}(1-z / w)^{n}$, and apply the binomial theorem as before.

Because we only need power series in three variables in this paper, we will not formulate a completely general theory. Instead, we will explicitly enumerate the embeddings we will need, and we will check that our conventions are consistent.

Definition 1.1.1. (1) We consider power series only in the 6 fundamental coordinates $z, w, t$, $z-w, z-t$, and $w-t$.

(2) Given a fundamental coordinate such as $z$, we use the notation $V \mapsto V\left[\left[z^{ \pm 1}\right]\right], f \mapsto f(z)$ to denote the endofunctor on the category of complex vector spaces that takes a vector space $V$ to the space $V\left[\left[z^{ \pm 1}\right]\right]$ of integral formal power series in $z$ with coefficients in $V$, and takes a complex linear transformation $f$ to the corresponding map of power series induced by applying the transformation to each coefficient. Elements of $V\left[\left[z^{ \pm 1}\right]\right]$ can be identified with set-theoretic maps $\mathbb{Z} \rightarrow V$, by identifying the coefficient of $z^{n}$ with the image of $n \in \mathbb{Z}$ under the map.

(3) Given an algebraically independent set of fundamental coordinates, we may iterate this functor to get spaces like $V\left[\left[z^{ \pm 1},(z-w)^{ \pm 1},(w-t)^{ \pm 1}\right]\right]$, in this case identifiable with the space of set-theoretic maps $\mathbb{Z}^{3} \rightarrow V$. All other spaces we consider will be identified as subspaces of these.

(4) The formal Taylor series functor $V \mapsto V[[z]]$ takes a vector space $V$ to the space of formal Taylor series in $z$ with coefficients in $V$, which can be identified with the set of maps $\mathbb{Z} \rightarrow V$ with support in $\mathbb{Z}_{\geq 0}$. Similarly, the polynomial functor $V \mapsto V[z]$ yields the subspace whose elements correspond to maps that are finitely supported, with support in $\mathbb{Z}_{\geq 0}$.

(5) The formal Laurent series functor $V \mapsto V((z))$ yields the space whose elements correspond to maps $\mathbb{Z} \rightarrow V$ supported on $\mathbb{Z}$, with support that is bounded from below. Note that $V((z))((w))$ and $V((w))((z))$ do not form equal subspaces of $V\left[\left[z^{ \pm 1}, w^{ \pm 1}\right]\right]$, since the powers of $w$ in the former space are bounded below, and the powers of $z$ in the latter space are bounded below, but not vice versa.

(6) Partial derivative operators on series spaces act on individual fundamental coordinates. To clarify by example, the operator $\partial_{w}$, as defined on $V((w))((z-w))$, takes $w^{m}(z-w)^{n}$ to $m w^{m-1}(z-w)^{n}$, not to $m w^{m-1}(z-w)^{n}-n w^{m}(z-w)^{n-1}$.

The reader should note that we may simplify the notation for maps when it is convenient to do so. For example, if $L(-1): V \rightarrow V$ is a linear endomorphism of a vector space, we may denote the corresponding endomorphism on $z^{n} V[[z]]$ by $L(-1)$ instead of $z^{n} L(-1)[[z]]$. We apologize for any confusion this may cause.

Definition 1.1.2. Because we are only using integral powers in this paper, we will not impose strong ordering conventions. However, we will prefer $z-w$ to $-(w-z)$, so we will write $V((z-w))$ instead of $V((w-z))$, even though they are the same space. We treat $w-t$ and $z-t$ similarly, i.e., one may think of this as an ordering $z \succ w \succ t$. We obtain the following conventions.

(1) $(z-w)^{n}=z^{n}(1-w / z)^{n} \in z^{n} \mathbb{C}\left[z^{-1}\right][[w]] \subset \mathbb{C}((z))((w))$, but we expand $(z-w)^{n}$ in $w^{n} \mathbb{C}\left[w^{-1}\right][[z]] \subset \mathbb{C}((w))((z))$ as $(-1)^{n} w^{n}(1-z / w)^{n}$.

(2) We expand $w^{n}$ in $z^{n} \mathbb{C}\left[z^{-1}\right][[z-w]] \subset \mathbb{C}((z))((z-w))$ as $z^{n}\left(1-\frac{z-w}{z}\right) n$, but we expand $z^{n}$ in $w^{n} \mathbb{C}\left[w^{-1}\right][[z-w]] \subset \mathbb{C}((w))((z-w))$ as $w^{n}\left(1+\frac{z-w}{w}\right)^{n}$. 
(3) We expand $(z-t)^{n}$ in $(w-t)^{n} \mathbb{C}\left[(w-t)^{-1}\right][[z-w]] \subset \mathbb{C}((w-t))((z-w))$ as $(w-t)^{n}\left(1+\frac{z-w}{w-t}\right)^{n}$. Similarly, we expand $(z-w)^{n}$ in $(z-t)^{n} \mathbb{C}\left[(z-t)^{-1}\right][[w-t]] \subset \mathbb{C}((z-t))((w-t))$ as $(z-t)^{n}\left(1-\frac{w-t}{z-t}\right)^{n}$

(4) We expand $(w-t)^{n}$ as $(-1)^{n}(z-w)^{n}\left(1-\frac{z-t}{z-w}\right)^{n}$ in $(z-w)^{n} \mathbb{C}\left[(z-w)^{-1}\right][[z-t]] \subset \mathbb{C}((z-$ $w))((z-t))$.

We define some functors from the category of vector spaces to itself using a subscript convention. Geometrically, the subscripts describe boundary strata in a partial compactification of a moduli space of genus zero curves with marked points in the following sense: We will consider objects labeled by $i, j, k$, and $\ell$ inserted at complex points $z, w, t$, and 0 , respectively. However, when two points collide, a complex line "bubbles off" at the place of their collision, and we more or less view the two insertions as lying at the same point in $\mathbb{C}$ (which, by convention, we have chosen to be the point attached to the latter label), but at different points in the "bubble". The corresponding vector spaces will be formal Laurent power series in the differences, and we enclose the two labels in parentheses (but we omit the parentheses enclosing the whole subscript). For example, we have the following six formal Laurent series spaces in the fundamental variables: $V_{k \ell}=V((t)), V_{j \ell}=V((w))$, $V_{i \ell}=V((z)), V_{i k}=V((z-t)), V_{i j}=V((z-w))$, and $V_{j k}=V((w-t))$. When three or more points collide, we take formal Taylor series in the differences, and invert all of the fundamental coordinates that appear - see the next definition. We use square brackets to indicate when a set of indices may be freely permuted without changing the resulting space.

Definition 1.1.3. For any vector space $V$, we define $V[[z, w]]\left[z^{-1}, w^{-1},(z-w)^{-1}\right]$ as the subspace of $V((z))((w))$ in which multiplication by a sufficiently large integer power of $z w(z-w)$ yields an element of $V[[z, w]]$. This yields a subfunctor of $V \mapsto V((z))((w))$. We write $V_{[i j] \ell}=$ $V[[z, w]]\left[z^{-1}, w^{-1},(z-w)^{-1}\right]$, and the embedding $V[[z, w]]\left[z^{-1}, w^{-1},(z-w)^{-1}\right] \rightarrow V((z))((w))$ is written $V_{[i j] \ell} \rightarrow V_{i(j \ell)}$. Here, the square brackets in the subscript indicate that switching $z$ and $w$ yields a naturally isomorphic functor.

Lemma 1.1.4. The conventions listed above yield the following embeddings:

(1) $V_{[i j] \ell} \rightarrow V_{i(j \ell)}$, meaning $V[[z, w]]\left[z^{-1}, w^{-1},(z-w)^{-1}\right] \rightarrow V((z))((w))$

(2) $V_{[i j] \ell} \rightarrow V_{j(i \ell)}$, meaning $V[[z, w]]\left[z^{-1}, w^{-1},(z-w)^{-1}\right] \rightarrow V((w))((z))$

(3) $V_{[i j] \ell} \rightarrow V_{(i j) \ell}$, meaning $V[[z, w]]\left[z^{-1}, w^{-1},(z-w)^{-1}\right] \rightarrow V((w))((z-w))$

(4) $V_{[i j] \ell} \rightarrow V_{(j i) \ell}$, meaning $V[[z, w]]\left[z^{-1}, w^{-1},(z-w)^{-1}\right] \rightarrow V((z))((z-w))$

(5) $V_{[i j] k} \rightarrow V_{i(j k)}$, meaning $V[[z-w, w-t]]\left[(z-w)^{-1},(z-t)^{-1},(w-t)^{-1}\right] \rightarrow V((z-t))((w-t))$

(6) $V_{[i j] k} \rightarrow V_{(i j) k}$, meaning $V[[z-w, w-t]]\left[(z-w)^{-1},(z-t)^{-1},(w-t)^{-1}\right] \rightarrow V((w-t))((z-w))$

Proof. Omitted.

Remark 1.1.5. There are some additional expansions we need to consider when working with three variables at a time.

(1) We expand $(z-t)^{n}$ in $\mathbb{C}[[w, t]]\left[w^{-1}, t^{-1},(w-t)^{-1}\right]((z-w))$ as $((z-w)+(w-t))^{n}=$ $(w-t)^{n}\left(1+\frac{z-w}{w-t}\right)^{n}$. Our choice of $(w-t)$ instead of $w$ or $t$ as the distinguished invertible fundamental coordinate is due to the fact that it yields a binomial.

(2) Some trinomial expansions are equal but not obviously so. For example $\left(1-\frac{w}{z}-\frac{t}{z}\right)^{n}$ is equal to both $\left(1-\frac{t}{z}\right)^{n}\left(1-\frac{w}{z(1-t / z)}\right)^{n}$ and $\left(1-\frac{t(1+w / t)}{z}\right)^{n}$. These more complicated expressions may appear when composing two embeddings.

Lemma 1.1.6. Given a vector space $V$, define the following vector spaces:

(1) $V_{[i j k] \ell}=V[[z, w, t]]\left[z^{-1}, w^{-1}, t^{-1},(z-w)^{-1},(z-t)^{-1},(w-t)^{-1}\right]$

(2) $V_{i([j k] \ell)}=V((z))[[w, t]]\left[w^{-1}, t^{-1},(w-t)^{-1}\right]$

(3) $V_{[i j](k \ell)}=V[[z, w]]\left[z^{-1}, w^{-1},(z-w)^{-1}\right]((t))$ 
(4) $V_{[i(j k)] \ell}=V[[z, t]]\left[z^{-1}, t^{-1},(z-t)^{-1}\right]((w-t))$

(5) $V_{[(i j) k] \ell}=V[[w, t]]\left[w^{-1}, t^{-1},(w-t)^{-1}\right]((z-w))$

(6) $V_{([i j] k) \ell}=V((t))[[z-w, z-t]]\left[(z-w)^{-1},(z-t)^{-1},(w-t)^{-1}\right]$

(7) $V_{i(j(k \ell))}=V((z))((w))((t))$

(8) $V_{i((j k) \ell)}=V((z))((t))((w-t))$

(9) $V_{(i j)(k \ell)}=V((w))((t))((z-w))$ - a referee points out we may also use $V((w))((z-w))((t))$.

(10) $V_{(i(j k)) \ell}=V((t))((z-t))((w-t))$

(11) $V_{((i j) k) \ell}=V((t))((w-t))((z-w))$

Then, our conventions for expansions yield the following commutative diagram of natural transformations in vector spaces:

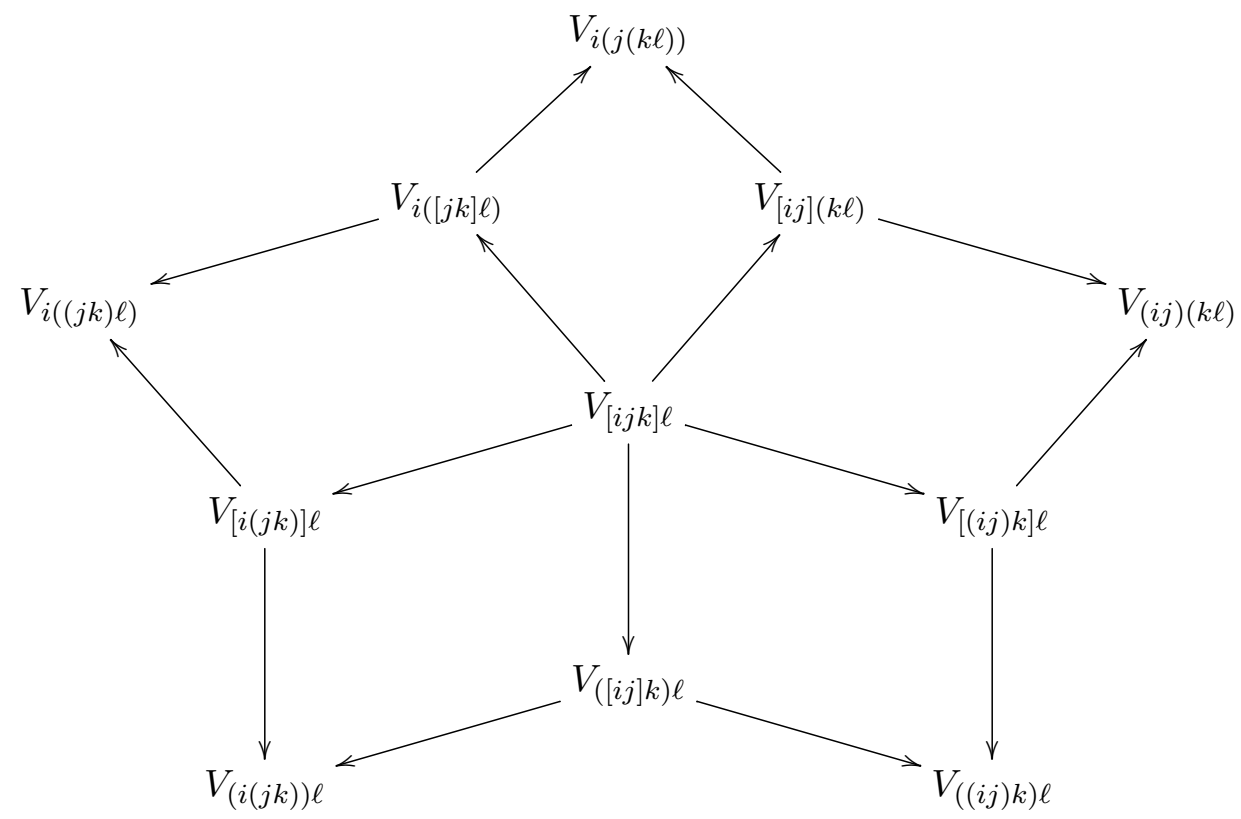

Proof. To check the commutativity of all quadrilaterals, it suffices to expand $v z^{a} w^{b} t^{c}(z-w)^{d}(z-$ $t)^{f}(w-t)^{g}$ along each of the compositions, where $v \in V$ and $a, b, c, d, f, g$ are integers. Since we may check validity after pulling back all natural transformations along the unique pointed linear map $(\mathbb{C}, 1) \mapsto(V, v)$, we may assume without loss of generality that $V$ is $\mathbb{C}$, and that $v=1$.

The inner five arrows yield:

(1) $z^{a+d+f}(1-w / z)^{d}(1-t / z)^{f} w^{b} t^{c}(w-t)^{g} \in V_{i([j k] \ell)}$

(2) $z^{a+f}(1-t / z)^{f} w^{b+g}(1-t / w)^{g}(z-w)^{d} t^{c} \in V_{[i j](k \ell)}$

(3) $z^{a} t^{b+c}\left(1-\frac{w-t}{t}\right)^{b}(z-t)^{d+f}\left(1-\frac{w-t}{z-t}\right)^{d}(w-t)^{g} \in V_{[i(j k)] \ell}$

(4) $w^{a+b}\left(1+\frac{z-w}{w}\right)^{a} t^{c}(w-t)^{f+g}\left(1+\frac{z-w}{w-t}\right)^{f}(z-w)^{d} \in V_{[(i j) k] \ell}$

(5) $t^{a+b+c}\left(1+\frac{z-t}{t}\right)^{a}\left(1+\frac{w-t}{t}\right)^{b}(z-w)^{d}(z-t)^{f}(w-t)^{g} \in V_{([i j] k) \ell}$

We obtain five comparisons:

(1) The top diamond yields $z^{a+d+f} w^{b+g} t^{c}(1-w / z)^{d}(1-t / z)^{f}(1-t / w)^{g}$.

(2) The right diamond compares $w^{a+b+f+g} t^{c}(z-w)^{d}\left(1+\frac{z-w}{w}\right)^{a+f}(1-t / w)^{g}\left(1-\frac{t}{w(1+(z-w) / w)}\right)^{f}$ to $w^{a+b+f+g}\left(1+\frac{z-w}{w}\right)^{a} t^{c}(1-t / w)^{f+g}\left(1+\frac{z-w}{w(1-t / w)}\right)^{f}(z-w)^{d}$. This amounts to comparing $\left(1+\frac{z-w}{w}\right)^{f}\left(1-\frac{t}{w(1+(z-w) / w)}\right)^{f}$ to $(1-t / w)^{f}\left(1+\frac{z-w}{w(1-t / w)}\right)^{f}$, and both are expansions of $\left(1-\frac{t}{w}+\frac{z-w}{w}\right)^{f} \in \mathbb{C}\left[w^{-1}\right][[t, z-w]]$.

(3) The left diamond compares $z^{a+d+f} t^{b+c}(w-t)^{g}(1-t / z)^{d+f}\left(1-\frac{w-t}{t}\right)^{b}\left(1-\frac{w-t}{z(1-t / z)}\right)^{d}$ to $z^{a+d+f} t^{b+c}(w-t)^{g}(1-t / z)^{f}\left(1-\frac{w-t}{t}\right)^{b}\left(1-\frac{t(1+(w-t) / t)}{z}\right)^{d}$. This amounts to comparing 
$(1-t / z)^{d}\left(1-\frac{w-t}{z(1-t / z)}\right)^{d}$ to $\left(1-\frac{t(1+(w-t) / t)}{z}\right)^{d}$, and both are expansions of $\left(1-\frac{t}{z}-\frac{w-t}{z}\right)^{d}$ in $\mathbb{C}\left[z^{-1}\right][[t, w-t]]$.

(4) The lower right diamond compares $t^{a+b+c}(w-t)^{f+g}(z-w)^{d}\left(1+\frac{w-t}{t}\right)^{a+b}\left(1+\frac{z-w}{t(1+(w-t) / t)}\right)^{a}(1+$ $\left.\frac{z-w}{w-t}\right)^{f}$ to $t^{a+b+c}(w-t)^{f+g}(z-w)^{d}\left(1+\frac{(z-w)+(w-t)}{t}\right)^{a}\left(1+\frac{w-t}{t}\right)^{b}\left(1-\frac{z-w}{w-t}\right)^{f}$. This amounts to comparing $\left(1+\frac{z-w}{t(1+(w-t) / t)}\right)^{a}\left(1+\frac{w-t}{t}\right)^{a}$ to $\left(1+\frac{(z-w)+(w-t)}{t}\right)^{a}$, and both are expansions of $\left(1+\frac{z-w}{t}+\frac{w-t}{t}\right)^{a}$ in $\mathbb{C}\left[t^{-1}\right][[z-w, w-t]]$.

(5) The lower left diamond yields $t^{a+b+c}(z-t)^{d+f}(w-t)^{g}\left(1+\frac{z-t}{t}\right)^{a}\left(1+\frac{w-t}{t}\right)^{b}\left(1-\frac{w-t}{z-t}\right)^{d}$.

Lemma 1.1.7. In addition to the vector spaces from Lemma 1.1.6, we define the following spaces:

(1) $V_{j((k i) \ell)}=V((w))((z))((z-t))$

(2) $V_{((j k) i) \ell}=V((z))((z-t))((w-t))$

(3) $V_{(j(k i)) \ell}=V((z))((z-w))((z-t))$

(4) $V_{j((k i) \ell)}=V((w))((z))((z-t))$

(5) $V_{j((i k) \ell)}=V((w))((t))((z-t))$

(6) $V_{(j(i k)) \ell}=V((t))((w-t))((z-t))$

(7) $V_{((j i) k) \ell}=V((t))((z-t))((z-w))$

(8) $V_{(k(i j)) \ell}=V((w))((w-t))((z-w))$

(9) $V_{((k i) j) \ell}=V((w))((z-w))((z-t))$

(10) $V_{((i k) j) \ell}=V((w))((w-t))((z-t))$

(11) $V_{(i(k j)) \ell}=V((w))((z-w))((w-t))$

(12) $V_{i((k j) \ell)}=V((z))((w))((w-t))$

(13) $V_{j([i k] \ell)}=V((w))[[z, t]]\left[z^{-1}, t^{-1},(z-t)^{-1}\right]$

(14) $V_{j(k i) \ell}=V[[z, w]]\left[z^{-1}, w^{-1},(z-w)^{-1}\right]((z-t))$

(15) $V_{j(i k) \ell}=V[[w, t]]\left[w^{-1}, t^{-1},(w-t)^{-1}\right]((z-t))$

(16) $V_{([j k] i) \ell}=V((z))[[z-w, z-t]]\left[(z-w)^{-1},(z-t)^{-1},(w-t)^{-1}\right]$

(17) $V_{([i k] j) \ell}=V((w))[[z-w, z-t]]\left[(z-w)^{-1},(z-t)^{-1},(w-t)^{-1}\right]$

(18) $V_{i(k j) \ell}=V[[z, w]]\left[z^{-1}, w^{-1},(z-w)^{-1}\right]((w-t))$

(19) $V_{i([j k] \ell)}=V((z))[[w, t]]\left[w^{-1}, t^{-1},(w-t)^{-1}\right]$

Then, we have the following commutative diagrams of embeddings:

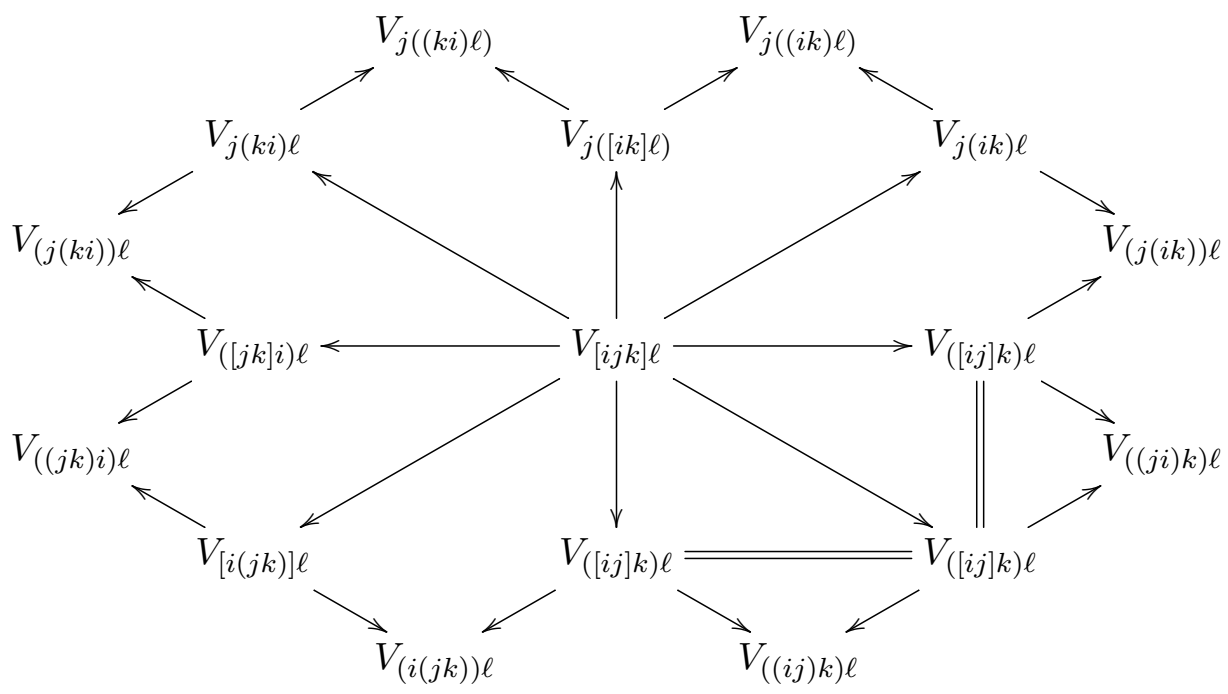




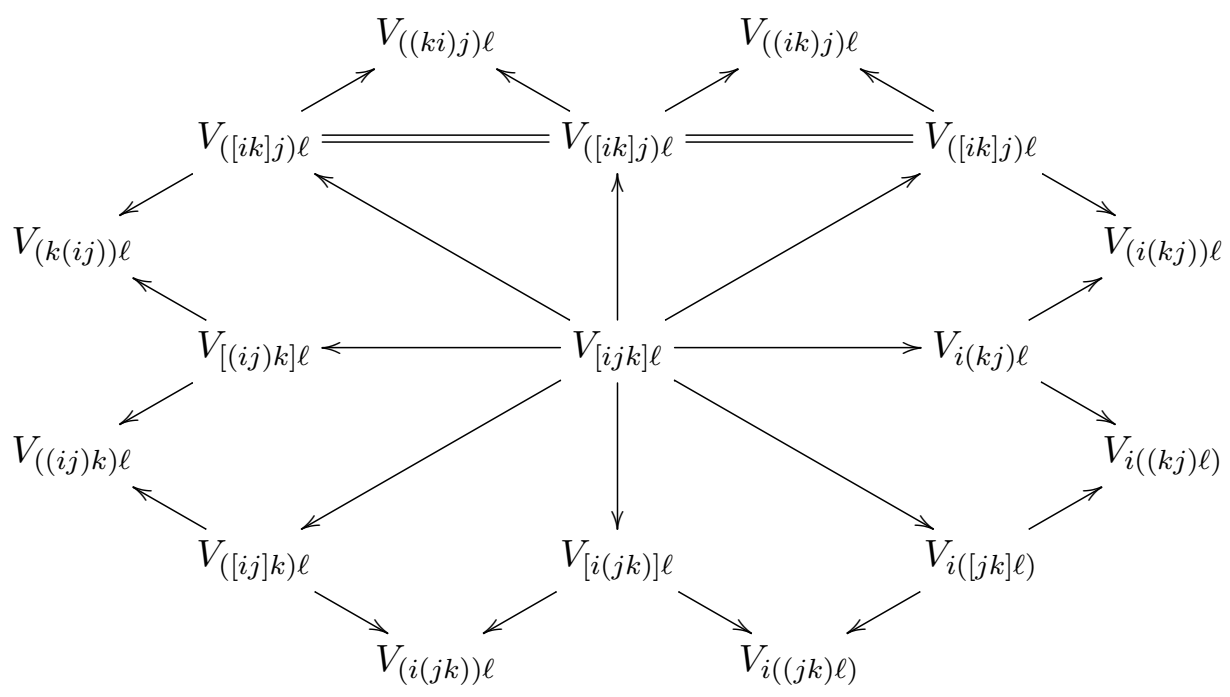

Proof. It suffices to expand the monomial $z^{a} w^{b} t^{c}(z-w)^{d}(z-t)^{f}(w-t)^{g}$ along each of the compositions. We will check one of the quadrilaterals, and leave the remaining 15 for the reader who wishes to repeat similar arguments.

In the first diagram, we start from $V_{[i j k] \ell}$ and go up to get $(-1)^{d} w^{b+d+g} z^{a} t^{c}(z-t)^{f}(1-z / w)^{d}(1-$ $t / w)^{g} \in V_{j([i k] \ell)}$. If we instead go to the upper left, we get $(-1)^{g} z^{a+c} w^{b}(z-w)^{d+g}\left(1-\frac{z-t}{z}\right)^{c}(1-$ $\left.\frac{z-t}{z-w}\right)^{g}(z-t)^{f} \in V_{j(k i) \ell}$. To check commutativity, it suffices to compare $(-1)^{d} w^{b+d+g} z^{a+c}(z-t)^{f}(1-$ $z / w)^{d}\left(1-\frac{z(1-(z-t) / z)}{w}\right)^{g}\left(1-\frac{z-t}{z}\right)^{c}$ with $(-1)^{d} w^{b+d+g} z^{a+c}(z-t)^{f}\left(1-\frac{z-t}{z}\right)^{c}\left(1-\frac{z-t}{-w(1-z / w)}\right)^{g}(1-z / w)^{d+g}$ in $V_{j((k i) \ell)}$.

Both evaluate to $(-1)^{d} w^{b+d+g} z^{a+c}(z-t)^{f}\left(1-\frac{z-t}{z}\right)^{c}(1-z / w)^{d}\left(1-\frac{z}{w}+\frac{z-t}{w}\right)^{g}$, so the quadrilateral with vertex $V_{j((k i) \ell)}$ commutes.

Lemma 1.1.8. (Formal Taylor theorem) If $f \in V\left[\left[z^{ \pm 1}\right]\right]$, then we have the following equalities in $V\left[\left[z^{ \pm 1}\right]\right][[w]]:$

(1) $f(z+w)=e^{w \partial_{z}} f(z)=\sum_{n \geq 0} \frac{(w)^{n}}{n !} \partial_{z}^{n} f(z)$.

(2) $f\left(z e^{w}\right)=e^{w z \partial_{z}} f(z)=\sum_{n>0} \frac{(w z)^{n}}{n !} \partial_{z}^{n} f(z)$.

The analogous result holds for any algebraically independent pair of fundamental coordinates.

Proof. See Frenkel-Lepowsky-Meurman 1988 Proposition 8.3.1.

Remark 1.1.9. One needs to be cautious about where elements live when using the formal Taylor theorem. For example, a naïve expansion of $\sum_{n \geq 0} \frac{(z-w)^{n}}{n !} \partial_{w}^{n}\left(w^{-1}\right)$ in $\mathbb{C}\{z, w\}$ yields a divergent sum for the coefficient of $w^{-1}$. However, as an element of $\mathbb{C}((w))[[z-w]] \subset \mathbb{C}((w))((z-w))$, we obtain the expansion of $z^{-1}$ as $\frac{1}{w} \sum_{n \geq 0}\left(\frac{z-w}{w}\right)^{n}$.

Lemma 1.1.10. (Frenkel-Ben-Zvi 2004 Remark 5.1.4) The map $f(z, z-w) \mapsto e^{(z-w) \partial_{w}} f(w, z-$ $w)=\sum_{n \geq 0} \frac{(z-w)^{n}}{n !} \partial_{w}^{n} f(w, z-w)$ describes an isomorphism $V((z))((z-w)) \rightarrow V((w))((z-w))$. Furthermore, it forms the horizontal arrow in the following commutative diagram:

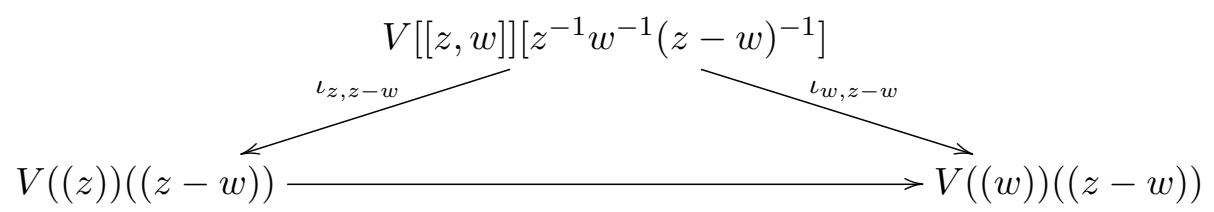

Proof. This follows straightforwardly from Lemma 1.1.8. 
1.2. Cohomology of a two-fold delooping. In MacLane 1952, Mac Lane introduces a cohomology theory of abelian groups that is distinct from ordinary group cohomology, and describes the explicit computation of the cohomology in low degree. Eilenberg explains in [Eilenberg 1952 that this cohomology theory is in fact the cohomology of a specific cell complex whose cohomology is isomorphic in low degree to a shift of the cohomology of the second Eilenberg-MacLane space. This claim is proved in Eilenberg-Mac Lane 1953 Theorem 20.4 (but with the definition of $A(\Pi, n$ ) shifted in degree from previous papers), where they do an explicit construction of $K(A, 2)$ via the diagonal of a two-fold Bar resolution. There are other methods for explicitly describing $K(A, 2)$, e.g., applying Dold-Kan correspondence to a homologically shifted abelian group (a freely available reference for this is Stacks-Project Theorem 019G), but the Eilenberg-Mac Lane complex happens to be the most convenient for our purposes.

Definition 1.2.1. ([MacLane 1952]) Let $A$ be an abelian group. The Eilenberg-Mac Lane cochain complex with coefficients in $\mathbb{C}^{\times}$has the following description in low degree:

$$
K^{1} \stackrel{d^{1}}{\longrightarrow} K^{2}=\left\{f: A \times A \rightarrow \mathbb{C}^{\times}\right\} \stackrel{d^{2}}{\longrightarrow} K^{3}=\left\{(F, \Omega): A^{\oplus 3} \times A^{\oplus 2} \rightarrow \mathbb{C}^{\times}\right\} \stackrel{d^{3}}{\longrightarrow} \cdots
$$

where:

(1) $K^{1}=\left\{\phi: A \rightarrow \mathbb{C}^{\times}\right\}$and $K^{2}=\left\{f: A \times A \rightarrow \mathbb{C}^{\times}\right\}$are groups of set-theoretic maps.

(2) $K^{3}=\left\{(F, \Omega): A^{\oplus 3} \times A^{\oplus 2} \rightarrow \mathbb{C}^{\times}\right\}$is a group of pairs of set-theoretic maps.

(3) The map $d^{1}: K^{1} \rightarrow K^{2}$ is given by the usual group cohomology coboundary $\phi \mapsto d^{1} \phi$, defined by $d^{1} \phi(i, j)=\frac{\phi(j) \phi(i)}{\phi(i+j)}$.

(4) The map $d^{2}: K^{2} \rightarrow K^{3}$ is given by the group cohomology coboundary together with the antisymmetrizer: $d^{2} f(i, j, k ; \ell, m)=\left(\frac{f(j, k) f(i, j+k)}{f(i+j, k) f(i, j)}, \frac{f(\ell, m)}{f(m, \ell)}\right)$.

(5) The map $d^{3}: K^{3} \rightarrow K^{4}$ vanishes if and only if the following conditions are satisfied:

(a) $F(i, j, k) F(i, j+k, \ell) F(j, k, \ell)=F(i+j, k, \ell) F(i, j, k+\ell)$ for all $i, j, k, \ell \in A$

(b) $F(i, j, k)^{-1} \Omega(i, j+k) F(j, k, i)^{-1}=\Omega(i, j) F(j, i, k)^{-1} \Omega(i, k)$

(c) $F(i, j, k) \Omega(i+j, k) F(k, i, j)=\Omega(j, k) F(i, k, j) \Omega(i, k)$

Elements annihilated by $d^{n}$ are called abelian $n$-cocycles, and an abelian $n$-cocycle lies in the $n$th abelian cohomology class.

Remark 1.2.2. The reader should be aware that elements of $n$-th abelian cohomology are identified with elements of $H^{n+1}\left(K(A, 2), \mathbb{C}^{\times}\right)$. That is, there is a shift in degrees.

Lemma 1.2.3. (MacLane 1952 Theorem 3) If $(F, \Omega)$ is an abelian 3-cocycle, then the map $Q$ : $A \rightarrow \mathbb{C}^{\times}$defined by $i \mapsto \Omega(i, i)$ is a quadratic form, i.e., $Q(i)=Q(-i)$ for all $i \in A$, and $\frac{Q(i+j+k) Q(i) Q(j) Q(k)}{Q(i+j) Q(i+k) Q(j+k)}=1$ for all $i, j, k \in A$. Furthermore, the trace map $(F, \Omega) \mapsto(i \mapsto \Omega(i, i))$ induces a bijection from $H^{4}\left(K(A, 2), \mathbb{C}^{\times}\right)$to the set of $\mathbb{C}^{\times}$-valued quadratic forms on $A$.

Proof. This is proved as Eilenberg-Mac Lane 1954, theorem 26.1.

Lemma 1.2.4. For any abelian group $A$, we have $H_{a b}^{2}\left(A, \mathbb{C}^{\times}\right) \cong H^{3}\left(K(A, 2), \mathbb{C}^{\times}\right)=0$.

Proof. The isomorphism between $H^{3}\left(K(A, 2), \mathbb{C}^{\times}\right)$and the group $\operatorname{Ext}_{\text {abel }}\left(A, \mathbb{C}^{\times}\right)$of abelian extensions of $\mathbb{C}^{\times}$by $A$ is said to be "well-known" in section 26 of [Eilenberg-Mac Lane 1954 (where the notation $\operatorname{Extabel}\left(A, \mathbb{C}^{\times}\right)$is used), and "can be explicitly computed" in [MacLane 1952] (where the confusing notation $\operatorname{Ext}\left(A, \mathbb{C}^{\times}\right)$is used). The group is trivial because the divisible property of $\mathbb{C}^{\times}$ makes any abelian extension split.

Definition 1.2.5. Let $A$ be an abelian group.

(1) Let $\eta: A \times A \rightarrow \mathbb{C}^{\times}$be a function, viewed as either a group cohomology 2-cochain or an abelian 2-cochain. We say that $\eta$ is normalized if $\eta(0, i)=\eta(i, 0)=1$ for all $i \in A$. 
(2) Let $F: A \times A \times A \rightarrow \mathbb{C}^{\times}$be a function, viewed as a group cohomology 3-cochain. We say that $F$ is normalized if $F(0, i, j)=F(i, 0, j)=F(i, j, 0)=1$ for all $i, j \in A$.

(3) We say that an abelian 3-cochain $(F, \Omega)$ is normalized if $F$ is normalized as a group cohomology 3 -cochain, and $\Omega$ is normalized as a 2 -cochain.

Lemma 1.2.6. All cohomology classes for the Eilenberg-Mac Lane model of $K(A, 2)$ in degree at most 4 are represented by normalized cochains. Normalized cocycles in an abelian cohomology class of degree at most 4 have a transitive action by differentials of normalized cochains.

Proof. Omitted - this is a straightforward computation.

Lemma 1.2.7. Let $B \subset A$ be a subgroup, and suppose we are given a $\mathbb{C}^{\times}$-valued quadratic form $i \mapsto \Omega(i, i)$ that is constant on cosets of $B$ in $A$. Then this quadratic form is pulled back from $a$ $\mathbb{C}^{\times}$-valued quadratic form on $A / B$, and there is a representing normalized abelian 3-cocycle $(F, \Omega)$ pulled back from $A / B$.

Proof. Omitted - this is also straightforward in light of 1.2 .3 .

Lemma 1.2.8. Let $Q: A \rightarrow \mathbb{C}^{\times}$be a quadratic form that takes values in \pm 1 . Then $Q$ is identically 1 on the subgroup $2 A=\{i+i \mid i \in A\}$, and constant on cosets of $2 A$ in $A$.

Proof. Both claims follow from the cube axiom: $Q(a+b+c) Q(a) Q(b) Q(c) / Q(a+b) Q(a+c) Q(b+c)=$ 1. For the first, we set $a=b=-c$, so $Q(2 a)=Q(a)^{4}=1$. For the second, we set $a=b$, so $Q(2 a+c)=Q(c)$.

The reader might want to know why degree 4 cohomology of $K(A, 2)$ naturally arises in this paper. Perhaps a good answer is that this cohomology group classifies braided tensor category structures on the abelian category of $A$-graded vector spaces (as shown in [Joyal-Street 1986]), and we will build something like a singular commutative ring in such a category.

\subsection{Structured vertex algebras.}

Definition 1.3.1. Given a tensor product $A_{1} \otimes \cdots \otimes A_{n}$ of complex vector spaces and a permutation $\sigma \in S_{n}$, we write $\tau_{\sigma}$ to indicate the linear isomorphism $A_{1} \otimes \cdots \otimes A_{n} \rightarrow A_{\sigma(1)} \otimes \cdots \otimes A_{\sigma(n)}$ determined by the assignment $a_{1} \otimes \cdots \otimes a_{n} \mapsto a_{\sigma(1)} \otimes \cdots \otimes a_{\sigma(n)}$, where $a_{i} \in A_{i}$ for all $1 \leq i \leq n$. Given a set $S$ of maps $A_{1} \otimes \cdots \otimes A_{n} \rightarrow B$, we write $\tau_{\sigma}^{*} S$ to denote the set whose elements are maps $A_{\sigma^{-1}(1)} \otimes \cdots \otimes A_{\sigma^{-1}(n)} \rightarrow B$ given by precomposing elements of $S$ with $\tau_{\sigma}$.

Definition 1.3.2. A vertex algebra is a tuple $\left(V, \mathbf{1}, L(-1), m_{z}\right)$, where:

(1) $V$ is a vector space.

(2) $\mathbf{1} \in V$ is an "identity" or "vacuum" element.

(3) $L(-1) \in \operatorname{End}(V)$ is a "translation" operator.

(4) $m_{z}: V \otimes V \rightarrow V((z))$ is a linear "multiplication" map.

These data are required to satisfy the following conditions:

(1) $m_{z}(\mathbf{1} \otimes v)=v z^{0}$ and $m_{z}(v \otimes \mathbf{1}) \in v z^{0}+z V[[z]]$ for all $v \in V$.

(2) $L(-1) m_{z}(u \otimes v)-m_{z}(u \otimes L(-1) v)=\frac{d}{d z} m_{z}(u \otimes v)$ for all $u, v \in V$. Here, the first $L(-1)$ is an abbreviation for $L(-1)((z))$. 
(3) The following diagram commutes:

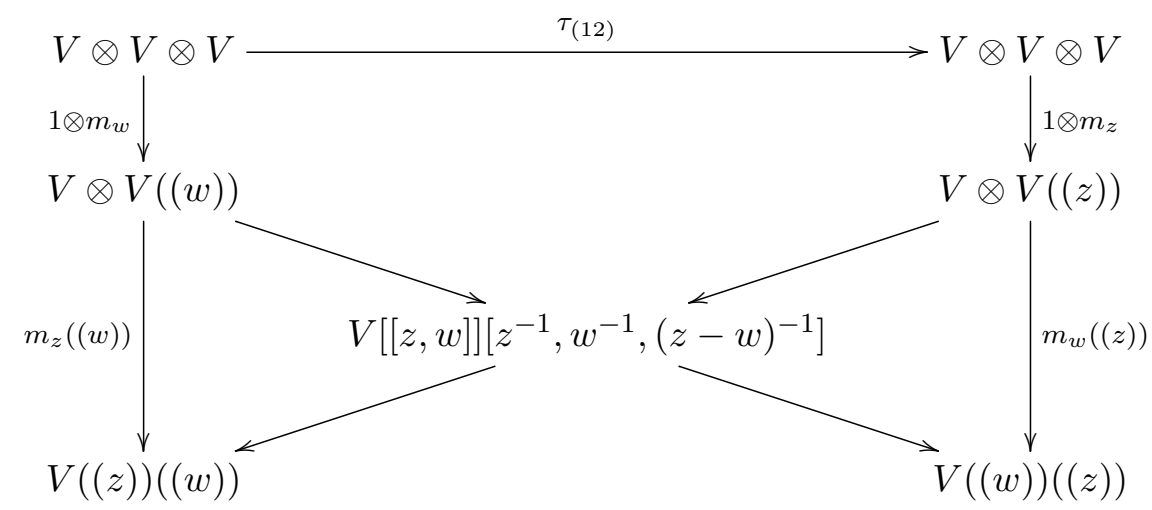

That is, the map $m_{z}((w)): V \otimes V((w)) \rightarrow V((z))((w))\left(\right.$ resp. $m_{w}((z)): V \otimes V((z)) \rightarrow$ $V((w))((z)))$ factors through the natural injection $V_{[i j] \ell} \rightarrow V_{i(j \ell)}\left(\right.$ resp. $\left.V_{[i j] \ell} \rightarrow V_{j(i \ell)}\right)$ given in Lemma 1.1.4.

Remark 1.3.3. This looks slightly different from the definitions presented elsewhere (e.g., Kac 1997] section 4.9). One typically has a map $Y: V \rightarrow($ End $V)\left[\left[z^{ \pm 1}\right]\right]$ in place of the map $m_{z}: V \otimes V \rightarrow$ $V((z))$ - it is straightforward to show that we obtain the original axioms by replacing $m_{z}(u \otimes v)$ with $Y(u, z) v$.

We introduce some symmetries that are commonly used in the vertex algebra literature. These are known as "worldsheet symmetries" in the physics literature.

Definition 1.3.4. A weighted (or $\mathbb{Z}$-graded) vertex algebra is a vertex algebra equipped with an operator $L(0): V \rightarrow V$ satisfying:

(1) $L(0)$ acts semisimply on $V$ with integer eigenvalues, so that $V=\bigoplus_{n \in \mathbb{Z}} V_{n}$ is an eigenvalue decomposition, where $L(0) v=n v$ for all $v \in V_{n}$.

(2) $L(0) m_{z}(u \otimes v)-m_{z}(u \otimes L(0) v)=m_{z}(L(0) u \otimes v)+z m_{z}(L(-1) u \otimes v)$ for all $u, v \in V$.

(3) $[L(-1), L(0)]=-L(-1)$.

A Möbius (resp., quasi-conformal) vertex algebra is a weighted vertex algebra equipped with an operator $L(1)$ (resp., operators $\{L(i)\}_{i=-1}^{\infty}$ ) such that:

(1) For all $i, j \geq-1,[L(i), L(j)]=(i-j) L(i+j)$. In particular, the linear span of the operators $L(-1), L(0), L(1)$ (resp., $\{L(i)\}_{i=-1}^{\infty}$ ) has a Lie algebra structure.

(2) For all $i \geq-1, L(i) m_{z}(u \otimes v)-m_{z}(u \otimes L(i) v)=\sum_{j=0}^{i+1}\left(\begin{array}{c}i+1 \\ j\end{array}\right) z^{j} m_{z}(L(i-j) u \otimes v)$.

(3) The sub-Lie algebra spanned by $\left\{L_{i}\right\}_{i \geq 1}$ acts locally nilpotently on $V$.

A conformal vertex algebra of central charge $c \in \mathbb{C}$ is a weighted vertex algebra equipped with a distinguished element $\omega \in V_{2}$ such that the operators $\{L(n): V \rightarrow V\}_{n \in \mathbb{Z}}$ defined by $m_{z}(\omega \otimes v)=$ $\sum_{n \in \mathbb{Z}} L(n) v z^{-n-2}$ satisfy the Virasoro relations:

$$
[L(m), L(n)]=(m-n) L(m+n)+\frac{m^{3}-m}{12} \delta_{m+n, 0} c
$$

A vertex operator algebra is a conformal vertex algebra whose $L(0)$-eigenvalues are bounded below, and whose $L(0)$-eigenspaces are finite dimensional.

Lemma 1.3.5. For future reference, we include the following facts:

(1) For any element $v$ in a vertex algebra $V, m_{z}(v \otimes \mathbf{1})=e^{z L(-1)} v$. More generally, we have the skew-symmetry property: $m_{z}(u \otimes v)=e^{z L(-1)} m_{-z}(v \otimes u)=e^{z L(-1)} m_{-z}(v \otimes u)$. Also, we have $m_{z}(L(-1) u \otimes v)=\frac{d}{d z} m_{z}(u \otimes v)$. 
(2) Given a vertex algebra $V$, the following diagram commutes:

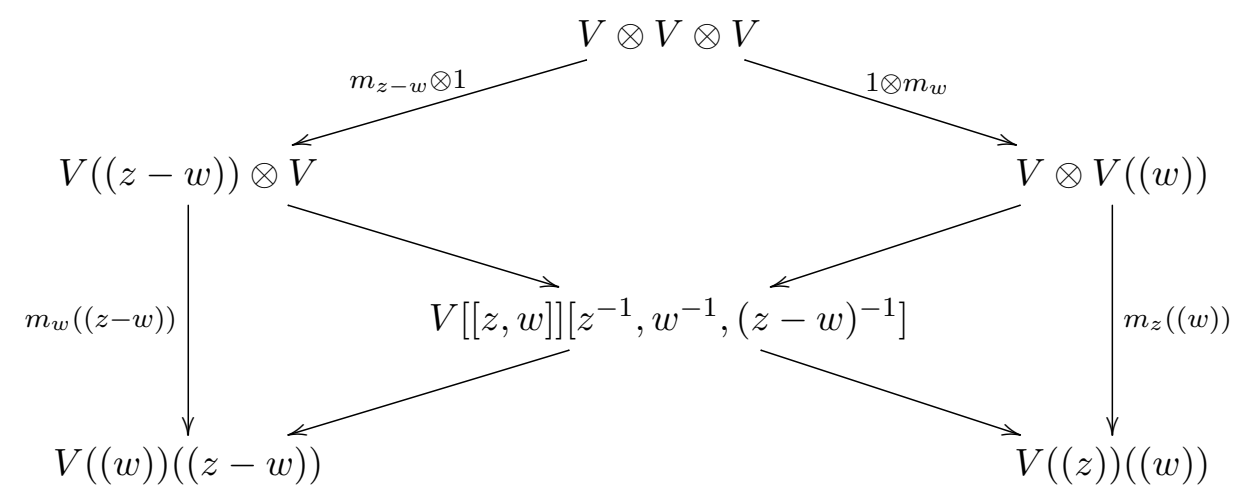

This is called the "associativity property".

(3) Let $V$ be a vertex algebra, let $n \geq 2$ be an integer, and let $\sigma \in S_{n}$ be a permutation. Then, the map $m_{z_{1}} \circ\left(1 \otimes m_{z_{2}}\right) \circ \cdots \circ\left(1 \otimes \cdots \otimes 1 \otimes m_{z_{n}}\right)$ from $V^{\otimes n} \otimes V$ to $V\left(\left(z_{n}\right)\right) \cdots\left(\left(z_{1}\right)\right)$ and the map $m_{z_{\sigma(1)}} \circ\left(1 \otimes m_{z_{\sigma(2)}}\right) \circ \cdots \circ\left(1 \otimes \cdots \otimes 1 \otimes m_{z_{\sigma(n)}}\right) \circ\left(\tau_{\sigma} \otimes 1\right)$ from $V^{\otimes n} \otimes V$ to $V\left(\left(z_{\sigma(n)}\right)\right) \cdots\left(\left(z_{\sigma(1)}\right)\right)$ factor through equal maps from $V^{\otimes n} \otimes V$ to $V\left[\left[z_{1}, \ldots, z_{n}\right]\right]\left[\left(\prod_{i=1}^{n}\left(z_{i} \prod_{j>i}\left(z_{i}-z_{j}\right)\right)\right)^{-1}\right]$.

Proof. The first claim is proved in Frenkel-Ben-Zvi 2004, Proposition 3.2.5 and Corollary 3.1.6.

The second claim is proved in, e.g., [Frenkel-Ben-Zvi 2004] Theorem 3.2.1.

The third claim is proved in [Frenkel-Ben-Zvi 2004] Theorem 4.5.1.

We will use the following finiteness condition near the end of the paper.

Definition 1.3.6. A vertex algebra is $C_{2}$-cofinite if the quotient $V /\left\{u_{-2} v \mid u, v \in V\right\}$ is a finite dimensional vector space.

Weighted vertex algebras that are $C_{2}$-cofinite tend to be quite well-behaved. In particular, their representation theory is highly controlled by certain finite dimensional associative algebras, although the statements of the theorems in question tend to assume vertex operator algebra structure unnecessarily.

\subsection{Modules and integral-weight intertwining operators.}

Definition 1.4.1. Let $V$ be a vertex algebra. A $V$-module is a vector space $M$ equipped with an action map act $t_{z}: V \otimes \rightarrow M((z))$ and an operator $L(-1)^{M}$, satisfying the following conditions:

(1) $\operatorname{act}_{z}(\mathbf{1} \otimes x)=x z^{0}$ for all $x \in M$

(2) $L(-1)^{M} \operatorname{act}_{z}(u \otimes x)-\operatorname{act}_{z}\left(u \otimes L(-1)^{M} x\right)=\operatorname{act}_{z}(L(-1) u \otimes x)$ for all $u \in V, x \in M$.

(3) The following diagram commutes:

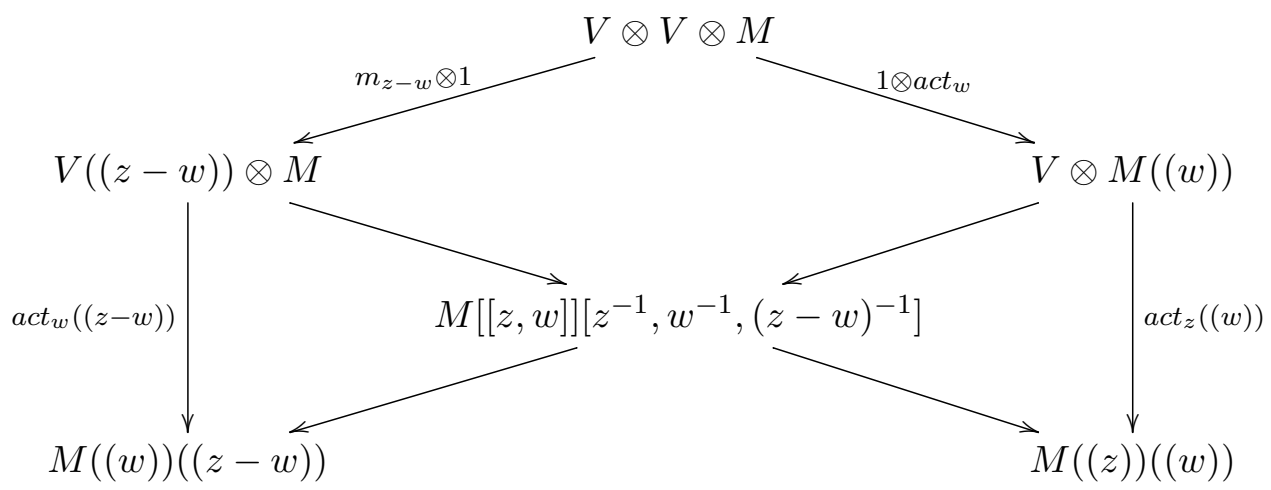


If $V$ is weighted (resp., Möbius, quasi-conformal), an integral-weight $V$-module is a module $M$ for the underlying vertex algebra, equipped with an operator $L(0)^{M}$ (resp., operators $L(0)^{M}$ and $L(1)^{M}$, operators $\left.\left\{L(i)^{M}\right\}_{i \geq-1}\right)$, satisfying the following conditions:

(1) $L(0)^{M}$ acts semisimply on $M$, so that $M=\bigoplus_{n \in \mathbb{Z}} M_{n}$ is an eigenvalue decomposition.

(2) For all $i, j \geq-1,\left[L(i)^{M}, L(j)^{M}\right]=(i-j) L(i+j)^{M}$.

(3) For all $i \geq-1$, any $u \in V$, and any $v \in M, L(i)^{M} \operatorname{act}_{z}(u \otimes v)-\operatorname{act}_{z}\left(u \otimes L(i)^{M} v\right)=$ $\sum_{j=0}^{i+1}\left(\begin{array}{c}i+1 \\ j\end{array}\right) z^{j} m_{z}(L(i-j) u \otimes v)$.

(4) The sub-Lie algebra spanned by $\left\{L_{i}\right\}_{i \geq 1}$ acts locally nilpotently on $V$.

If $V$ is a conformal vertex algebra of central charge $c$, an integral-weight $V$-module is an integralweight module $M$ for the underlying weighted vertex algebra, satisfying the additional condition that the operators $\left\{L(n)^{M}: M \rightarrow M\right\}_{n \in \mathbb{Z}}$ defined by $\operatorname{act}_{z}(\omega \otimes x)=\sum_{n \in \mathbb{Z}} L(n)^{M} x z^{-n-2}$ yield a representation of the Virasoro algebra of central charge $c$. If $V$ is a vertex operator algebra, then an integral-weight $V$-modules is an integral-weight module $M$ for the underlying weighted vertex algebra, satisfying the additional conditions that the $L(0)$-eigenvalues are bounded below, and the $L(0)$-eigenspaces are finite dimensional.

We will use the following near the end of the paper.

Definition 1.4.2. A vertex operator algebra $V$ is rational if every $V$-module (not just those of integral weight) is completely reducible. $V$ is regular if it is rational and $C_{2}$-cofinite.

Definition 1.4.3. Let $V$ be a vertex algebra, and let $M_{1}, M_{2}, M_{3}$ be $V$-modules. An integralweight intertwining operator of type $\left(\begin{array}{c}M_{3} \\ M_{1} M_{2}\end{array}\right)$ is a map $I_{z}: M_{1} \otimes M_{2} \rightarrow M_{3}((z))$ satisfying the following conditions:

(1) Translation covariance: $\frac{d}{d z} I_{z}(u \otimes v)=I_{z}\left(L(-1)^{M_{1}} u \otimes v\right)=L(-1)^{M_{3}} I_{z}(u \otimes v)-I_{z}(u \otimes$ $\left.L(-1)^{M_{2}} v\right)$ for any $u \in M_{1}$ and $v \in M_{2}$.

(2) $V$-compatibility: The maps

(a) $\operatorname{act}_{z}((w)) \circ\left(1 \otimes I_{w}\right): V \otimes M_{1} \otimes M_{2} \rightarrow M_{3}((z))((w))$

(b) $I_{w}((z-w)) \circ\left(\operatorname{act}_{z-w} \otimes 1\right): V \otimes M_{1} \otimes M_{2} \rightarrow M_{3}((w))((z-w))$

(c) $I_{w}((z)) \circ\left(1 \otimes a c t_{z}\right): M_{1} \otimes V \otimes M_{2} \rightarrow M_{3}((w))((z))$

factor through the space $M_{3}[[z, w]]\left[z^{-1}, w^{-1},(z-w)^{-1}\right]$ where they coincide.

If $V$ is weighted (resp., Möbius, quasi-conformal), and $M_{1}, M_{2}, M_{3}$ are integral-weight $V$-modules in the corresponding sense, then an integral-weight intertwining operator of type $\left(\begin{array}{c}M_{3} \\ M_{1} M_{2}\end{array}\right)$ is an integral-weight intertwining operator for the underlying vertex algebra modules, satisfying the compatibility:

$$
L(i)^{M_{3}} I_{z}(u \otimes v)-I_{z}\left(u \otimes L(i)^{M_{2}} v\right)=\sum_{j=0}^{i+1}\left(\begin{array}{c}
i+1 \\
j
\end{array}\right) z^{j} I_{z}\left(L(i-j)^{M_{1}} u \otimes v\right)
$$

for all applicable operators $L(i)$.

Remark 1.4.4. The $V$-compatibility condition has equivalent variants in the literature, e.g., it is a straightforward translation of the commutativity and associativity given in Dong-Lepowsky 1993 Propositions 11.4 and 11.5, and by Remark 11.6 this is equivalent to the Jacobi identity:

$$
\begin{gathered}
z_{0}^{-1} \delta\left(\frac{z_{1}-z_{2}}{z_{0}}\right) Y^{M_{3}}\left(a, z_{1}\right) I\left(b, z_{2}\right) c-z_{0}^{-1} \delta\left(\frac{z_{2}-z_{1}}{z_{0}}\right) I\left(b, z_{2}\right) Y^{M_{1}}\left(a, z_{1}\right) c \\
=z_{2}^{-1} \delta\left(\frac{z_{1}-z_{0}}{z_{2}}\right) I\left(Y\left(a, z_{0}\right) b, z_{2}\right) c .
\end{gathered}
$$

Lemma 1.4.5. Let $V$ be a vertex algebra. Given an integral-weight intertwining operator $I_{z}$ : $M_{1} \otimes M_{2} \rightarrow M_{3}((z))$, the map $I_{z}^{*}: M_{2} \otimes M_{1} \rightarrow M_{3}((z))$ given by $I_{z}^{*}(v \otimes u)=e^{z L(-1)} I_{-z}(u \otimes v)$ is an 
integral-weight intertwining operator. In particular, for any $V$-module $M$, the map act $t_{z}^{*}: M \otimes V \rightarrow$ $M((z))$ defined by act $t_{z}^{*}(x \otimes v)=e^{z L(-1)} a c t_{-z}(v \otimes x)$ for all $v \in V$ and $x \in M$, is an integral-weight intertwining operator. The same statements hold if $V$ is weighted, Möbius, or (quasi-)conformal.

Proof. In the conformal case, this is Proposition 5.4.7 in Frenkel-Huang-Lepowsky 1993]. In the Möbius case, this is a special case of Proposition 3.4.4 in [Huang-Lepowsky-Zhang 2007], where $I_{z}$ is non-logarithmic, and $r=0$. The argument in their proof does not use the Möbius structure that is assumed to exist, except where it pertains to $L(i)$-compatibility conditions of $I_{z}^{*}$.

Remark 1.4.6. Lemma 1.4.5 implies the intertwining operator $a c t_{z}^{*}$, together with $a c t_{z}$, induces a "split square-zero extension" vertex algebra structure on $V \oplus M$.

Lemma 1.4.7. Let $V$ be a vertex algebra, and let $M_{1}$ and $M_{2}$ be $V$-modules. If $I_{z}: M_{1} \otimes M_{1} \rightarrow$ $M_{2}\{z\}$ is a nonzero integral-weight intertwining operator that is a constant multiple of $I_{z}^{*}$, then $I_{z}(u \otimes v)= \pm I_{z}^{*}(u \otimes v)$ for all $u, v \in M_{1}$.

Proof. We consider two cases, depending on whether $I_{z}$ is alternating.

First case: Suppose there exists $u \in M_{1}$ such that $I_{z}(u \otimes u) \neq 0$. We may write $I_{z}(u \otimes u)=$ $\sum_{n=0}^{\infty} g_{n+r} z^{n+r}$, with $g_{r}$ a nonzero element of $M_{2}$, and $r \in \mathbb{Z}$. Since $I_{z}^{*}(u \otimes u)=e^{z L(-1)} I_{-z}(u \otimes u)$, it suffices to check that the lowest-order term in the latter series is $(-1)^{r} g_{r} z^{r}$. By assumption, $I_{z}$ is proportional to $I_{z}^{*}$, and our calculation shows that the constant of proportionality is \pm 1 .

Second case: If for all $u \in M_{1}, I_{z}(u \otimes u)=0$, then bilinearity implies $I_{z}(u \otimes v)=-I_{z}(v \otimes u)$ for all $u, v \in M_{1}$. Write $I_{z}(u \otimes v)=\sum_{n=0}^{\infty} g_{n+r} z^{n+r}$, with $g_{r}$ a nonzero element of $M_{2}$, and $r \in \mathbb{Z}$. We note that:

$$
\begin{aligned}
I_{z}^{*}(u \otimes v) & =e^{z L(-1)} I_{-z}(v \otimes u) \\
& =-e^{z L(-1)} \sum_{n=0}^{\infty} g_{n+r}(-z)^{n+r}
\end{aligned}
$$

so the lowest order term is $-(-1)^{r} g_{r} z^{r}$. By assumption, $I_{z}$ is proportional to $I_{z}^{*}$, and our calculation shows that the constant of proportionality is \pm 1 .

Definition 1.4.8. Let $V$ be a vertex algebra equipped with an action of a group $G$ by vertex algebra automorphisms. A $G$-action on a $V$-module $M$ (as a vector space) is compatible with the $V$-module structure if the following conditions hold:

(1) For any element $g \in G, \operatorname{act}_{z}(g v \otimes g u)=\operatorname{gact}_{z}(v \otimes u)$ for all $v \in V$ and $u \in M$.

(2) For any $g \in G, L(-1) g=g L(-1)$.

(3) If $V$ and $M$ have additional worldsheet symmetry given by operators $\{L(i)\}$, then $L(i) g=$ $g L(i)$ for all applicable $i$ and all $g \in G$.

Given $V$-modules $M_{1}, M_{2}, M_{3}$ equipped with $G$-actions compatible with their $V$-module structures, an integral-weight intertwining operator $I_{z}: M_{1} \otimes M_{2} \rightarrow M_{3}((z))$ is $G$-equivariant if for any $g \in G$, $I_{z}(g u \otimes g v)=g I_{z}(u \otimes v)$ for all $u \in M_{1}$ and $v \in M_{2}$.

\section{OBstruction theORY}

Let $A$ be an abelian group, $V$ a vertex algebra, and $\left\{M_{i}\right\}_{i \in A}$ a set of $V$-modules, such that $V=M_{0}$. Suppose we are given one-dimensional vector spaces $\mathcal{I}_{i, j}^{i+j}$ whose elements are integralweight intertwining operators $m_{z}^{i, j}: M_{i} \otimes M_{j} \rightarrow M_{i+j}((z))$ for all $i, j \in A$. We are concerned with the following:

Question: Is it possible to choose nonzero elements in each $\mathcal{I}_{i, j}^{i+j}$ so that $\bigoplus_{i \in A} M_{i}$ is endowed with a vertex algebra structure? 
A priori, there is no reason for this to hold, because vertex algebras satisfy a locality property that requires compositions of our intertwining operators to satisfy some compatibilities, and we have not put in place any constraints on our maps. In particular, the locality axiom is equivalent to the commutativity of the following diagram for each $i, j, k \in A$ :

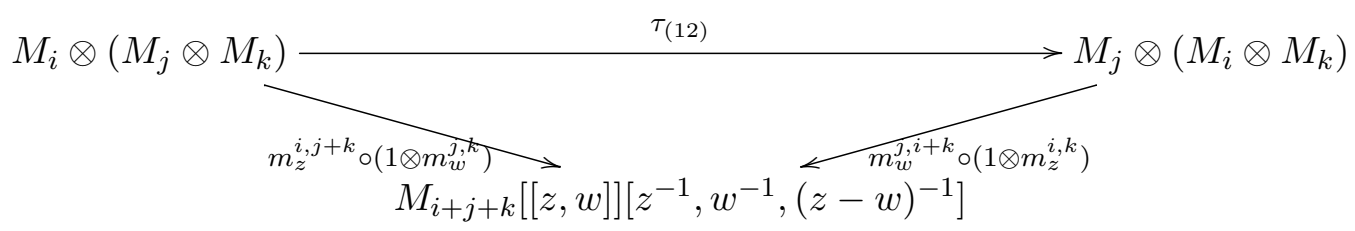

and we have not even imposed the condition that the two composite maps are proportional to each other.

To convert this condition on intertwining operators into a homological problem, we rephrase it in terms of an equivalent condition that concerns singular commutativity and associativity.

Since we are interested in constructing several types of vertex algebras (e.g., Möbius vertex algebras, quasi-conformal vertex algebras, vertex operator algebras), we will refrain from treating each case separately. Instead, we consider a datum $\mathcal{T}$, that is akin to a structured category of modules with certain spaces of integral-weight intertwining operators attached, but is used like a type. We won't be particularly precise about the properties of $\mathcal{T}$ that we need, but the results of this paper hold for any of the following examples:

(1) The unrestricted setting: A $\mathcal{T}$-vertex algebra $V$ is a vertex algebra, $V$-modules in $\mathcal{T}$ are modules for the vertex algebra $V, \mathcal{T}$-morphisms are $V$-module maps, and $\mathcal{T}$-intertwining operators are integral-weight intertwining operators.

(2) Worldsheet symmetry: A $\mathcal{T}$-vertex algebra $V$ is a weighted (resp. Möbius, quasiconformal, conformal) vertex algebra, $V$-modules in $\mathcal{T}$ are integral-weight $V$-modules (resp. with Möbius, quasiconformal, conformal structure), $\mathcal{T}$-morphisms are $\{L(i)\}$-equivariant module maps for all applicable operators $L(i)$, and $\mathcal{T}$-intertwining operators are integral-weight and compatible with the defined $L(i)$ operators.

(3) VOAs: A $\mathcal{T}$-vertex algebra $V$ is a vertex operator algebra, $V$-modules in $\mathcal{T}$ are integralweight $V$-modules in the VOA sense, i.e., with $L(0)$-spectrum bounded below and with finite multiplicity, morphisms are VOA-module maps, and intertwining operators are integralweight VOA intertwining operator maps.

(4) Strictly $G$-equivariant versions of the previous examples, where a group $G$ acts on $V$ by automorphisms, modules have $G$-action compatible with the action on $V$, and maps of modules and intertwining operators are $G$-equivariant. This example would be more useful if we allowed projective actions on modules, but in our final results, we would have to replace $G$ with a central extension, and the notation would become cumbersome.

In this language, our aim is to start with a $\mathcal{T}$-vertex algebra $V$, a collection of modules $\left\{M_{i}\right\}_{i \in A}$ in $\mathcal{T}$ with $V=M_{0}$, and build a $\mathcal{T}$-vertex algebra structure on $\bigoplus_{i \in A} M_{i}$ using $\mathcal{T}$-intertwining operators. Given a $\mathcal{T}$-vertex algebra $V$, and $V$-modules $M_{1}, M_{2}, M_{3}$ in $\mathcal{T}$, we write $I_{\mathcal{T}}\left(\begin{array}{c}M_{3} \\ M_{1}, M_{2}\end{array}\right)$ to denote the space of $\mathcal{T}$-intertwining operators $M_{1} \otimes M_{2} \rightarrow M_{3}((z))$. One may view the data making up $\mathcal{T}$ as a rather primitive version of the notion of multicategory or pseudo-tensor category, but in the long run, some kind of type theory may be a better fit for making this example-driven study into a set of general theorems. 
2.1. Associativity. In order for a choice of $\mathcal{T}$-intertwining operators to yield a $\mathcal{T}$-vertex algebra, it is necessary (but not sufficient) for the following associativity diagram to commute:

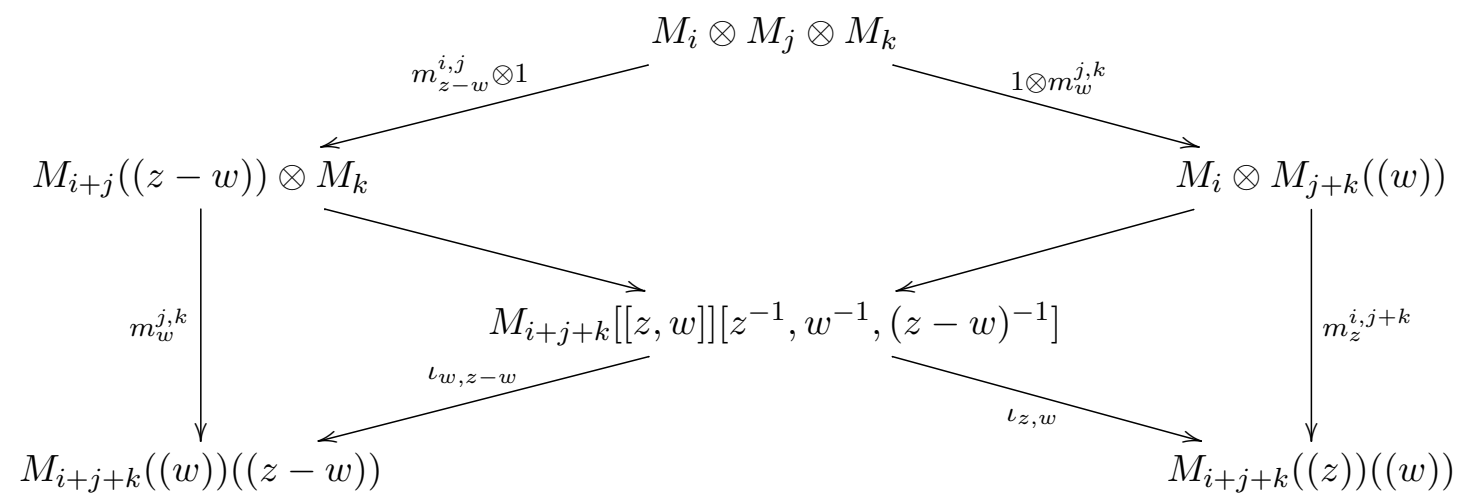

One way to approach the associativity condition is to assume the canonical composition operation on $\mathcal{I}_{i, j+k}^{i+j+k} \otimes \mathcal{I}_{j, k}^{j+k}$ and $\mathcal{I}_{i+j, k}^{i+j+k} \otimes \mathcal{I}_{i, j}^{i+j}$ yields elements in the same one dimensional space $\mathcal{I}_{i, j, k}^{i+j+k}$ of maps:

$$
M_{i} \otimes M_{j} \otimes M_{k} \rightarrow M_{i+j+k}[[z, w]]\left[z^{-1}, w^{-1},(z-w)^{-1}\right]
$$

If this holds, then for any choice of nonzero elements in each $\mathcal{I}_{i, j}^{i+j}$, the failure of the associativity diagram to commute is encoded by a set of nonzero constants $\left\{F(i, j, k) \in \mathbb{C}^{\times}\right\}_{i, j, k \in A}$. We may then hope that these constants can be adjusted to 1 by suitably rescaling our intertwining operators. To encode this more specifically, we have the following definition:

Definition 2.1.1. Let $V$ be a $\mathcal{T}$-vertex algebra, let $A$ be an abelian group, and let $\left\{M_{i}\right\}_{i \in A}$ be a set of $V$-modules in $\mathcal{T}$, such that $M_{0}=V$. We define a one dimensional $\mathcal{T}$-associativity datum to be a set $\left\{\mathcal{I}_{i, j}^{i+j}\right\}_{i, j \in A}$ of one-dimensional subspaces of $I_{\mathcal{T}}\left(\begin{array}{c}M_{i+j} \\ M_{i}, M_{j}\end{array}\right)$ (i.e., whose elements are integral-weight intertwining operators $\left.M_{i} \otimes M_{j} \rightarrow M_{i+j}((z))\right)$, such that:

(1) For each $i, j, k \in A$, there exists a one dimensional vector space $\mathcal{I}_{i, j, k}^{i+j+k}$ whose elements are maps $M_{i} \otimes M_{j} \otimes M_{k} \rightarrow M_{i+j+k}[[z, w]]\left[z^{-1}, w^{-1},(z-w)^{-1}\right]$, such that composition of intertwining operators, combined with the canonical injections from Lemma 1.1.4, induces isomorphisms from $\mathcal{I}_{i, j+k}^{i+j+k} \otimes \mathcal{I}_{j, k}^{j+k}$ to $\mathcal{I}_{i, j, k}^{i+j+k}$ and from $\mathcal{I}_{i+j, k}^{i+j+k} \otimes \mathcal{I}_{i, j}^{i+j}$ to $\mathcal{I}_{i, j, k}^{i+j+k}$.

(2) For any $i, j, k, \ell \in A$, there exists a one-dimensional space $\mathcal{I}_{i, j, k, \ell}^{i+j+k+\ell}$ whose elements are maps:

$M_{i} \otimes M_{j} \otimes M_{k} \otimes M_{\ell} \rightarrow M_{i+j+k+\ell}[[z, w, t]]\left[z^{-1}, w^{-1} t^{-1},(z-w)^{-1},(z-t)^{-1},(w-t)^{-1}\right]$

such that composition of intertwining operators, combined with one of the canonical injections from Lemma 1.1.6, induces an isomorphism from $\mathcal{I}_{i, j+k+\ell}^{i+j+k+\ell} \otimes \mathcal{I}_{j, k+\ell}^{j+k+\ell} \otimes \mathcal{I}_{k, \ell}^{k+\ell}$ to $\mathcal{I}_{i, j, k, \ell}^{i+j+k+\ell}$.

(3) The space $\mathcal{I}_{0, i}^{i}$ is spanned by the module structure map act $t_{z}^{i}: V \otimes M_{i} \rightarrow M_{i}((z))$.

(4) The space $\mathcal{I}_{i, 0}^{i}$ is spanned by the intertwining operator $\operatorname{act}_{z}^{i, *}: M_{i} \otimes V \rightarrow M_{i}((z))$ described in Lemma 1.4.5.

Given a one-dimensional $\mathcal{T}$-associativity datum, and any choice of nonzero intertwining operators $\left\{m_{z}^{i, j} \in \mathcal{I}_{i, j}^{i+j}\right\}_{i, j \in A}$, we define the function $F: A^{\oplus 3} \rightarrow \mathbb{C}^{\times}$by

$$
m_{z}^{i, j+k} \circ\left(1 \otimes m_{w}^{j, k}\right)=F(i, j, k) m_{w}^{i+j, k} \circ\left(m_{z-w}^{i, j} \otimes 1\right)
$$

Remark 2.1.2. In order for this definition to make sense, it is necessary to choose certain embeddings of vector spaces (or more precisely, natural transformations of power series endofunctors on $V e c t$ ), and Lemma 1.1.6 ensures this can be done consistently. 
Remark 2.1.3. It does not seem to be necessary to assume the existence of the spaces $\mathcal{I}_{i, j, k, \ell}^{i+j+k+\ell}$, as long as we assume that all of the modules $\left\{M_{i}\right\}_{i \in A}$ are well-behaved simple currents (see Section 3). However, without strong conditions, it is possible for the composition of nonzero intertwining operators to be zero.

Lemma 2.1.4. Given a one-dimensional $\mathcal{T}$-associativity datum $\left\{\mathcal{I}_{i, j}^{i+j}\right\}_{i, j \in A}$, the composition of intertwining operators induces isomorphisms between the following one-dimensional vector spaces, for all $i, j, k, \ell \in A$ :

$$
\begin{aligned}
\mathcal{I}_{i, j, k, \ell}^{i+j+k+\ell} & \cong \mathcal{I}_{i, j+k+\ell}^{i+j+k+\ell} \otimes \mathcal{I}_{j, k+\ell}^{j+k+\ell} \otimes \mathcal{I}_{k, \ell}^{k+\ell} \cong \mathcal{I}_{i, j, k+\ell}^{i+j+k+\ell} \otimes \mathcal{I}_{k, \ell}^{k+\ell} \\
& \cong \mathcal{I}_{i+j+k+\ell}^{i+j+k+\ell} \otimes \mathcal{I}_{k, \ell}^{k+\ell} \otimes \mathcal{I}_{i, j}^{i+j} \cong \mathcal{I}_{i+j+k+\ell}^{i+j+k+\ell} \otimes \mathcal{I}_{i, j}^{i+j} \\
& \cong \mathcal{I}_{i+j+k+\ell}^{i+j+k, \ell} \otimes \mathcal{I}_{i+j, k}^{i+j+k} \otimes \mathcal{I}_{i, j}^{i+j} \cong \mathcal{I}_{i+j+k+\ell}^{i+j+k, \ell} \otimes \mathcal{I}_{i, j, k}^{i+j+k} \\
& \cong \mathcal{I}_{i+j+k+\ell}^{i+j+k} \otimes \mathcal{I}_{i, j+k}^{i+j+k} \otimes \mathcal{I}_{j, k}^{j+k} \cong \mathcal{I}_{i, j+k+k}^{i+j+k+\ell} \otimes \mathcal{I}_{j, k}^{j+k} \\
& \cong \mathcal{I}_{i, j+k+\ell}^{i+j+k+\ell} \otimes \mathcal{I}_{j+k, \ell}^{j+k+\ell} \otimes \mathcal{I}_{j, k}^{j+k} \cong \mathcal{I}_{i, j+k+\ell}^{i+j+k+\ell} \otimes \mathcal{I}_{j, k, \ell}^{j+k+\ell}
\end{aligned}
$$

In particular, any element in one of the above spaces describes a composite of $\mathcal{T}$-intertwining operators that takes elements of $M_{i} \otimes M_{j} \otimes M_{k} \otimes M_{\ell}$ and factors through a canonical embedding from $M_{i+j+k+\ell}[[z, w, t]]\left[z^{-1}, w^{-1} t^{-1},(z-w)^{-1},(z-t)^{-1},(w-t)^{-1}\right]$.

Proof. The first isomorphism is the condition defining $\mathcal{I}_{i, j, k, \ell}^{i+j+k+\ell}$. The other isomorphisms follow from the conditions defining the set of spaces $\left\{\mathcal{I}_{i, j, k}^{i+j+k}\right\}_{i, j, k \in A}$. The fact that all of the maps factor through $M_{i+j+k+\ell}[[z, w, t]]\left[z^{-1}, w^{-1} t^{-1},(z-w)^{-1},(z-t)^{-1},(w-t)^{-1}\right]$, and hence the corresponding commutative diagram of embeddings given in Lemma 1.1.6. follows from the standard fact that if $f: U \rightarrow V$ is a linear map of vector spaces that factors as a composite of linear maps $U \rightarrow W \rightarrow V$, and $g$ is a constant multiple of $f$, then $g$ factors through $W$.

Lemma 2.1.5. Given a one-dimensional $\mathcal{T}$-associativity datum, and any choice of nonzero intertwining operators $\left\{m_{z}^{i, j} \in \mathcal{I}_{i, j}^{i+j}\right\}_{i, j \in A}$, the function $F$ satisfies the pentagon identity:

$$
F(i, j, k) F(i, j+k, \ell) F(j, k, \ell)=F(i+j, k, \ell) F(i, j, k+\ell)
$$

for any $i, j, k, \ell \in A$. In other words, the function $F$, viewed as a group cohomology 3-cochain, is in fact a 3-cocycle.

Proof. For any $i, j, k, \ell \in A$, let $u_{i} \in M_{i}, u_{j} \in M_{j}, u_{k} \in M_{k}$ and $u_{\ell} \in M_{\ell}$ satisfy:

$$
m_{z}^{i, j+k+\ell} \circ\left(1 \otimes m_{w}^{j, k+\ell}\right) \circ\left(1 \otimes 1 \otimes m_{t}^{k, \ell}\right)\left(u_{i} \otimes u_{j} \otimes u_{k} \otimes u_{\ell}\right) \neq 0 \in M_{i+j+k+\ell}((z))((w))((t)) .
$$

For convenience, we write $M^{\prime}=M_{i+j+k+\ell}[[z, w, t]]\left[z^{-1}, w^{-1} t^{-1},(z-w)^{-1},(z-t)^{-1},(w-t)^{-1}\right]$. By the existence of $\mathcal{I}_{i, j, k, \ell}^{i+j+k+\ell}$, a satisfactory choice of elements $\left(u_{i}, u_{j}, u_{k}, u_{\ell}\right)$ exists, and the map $m_{z}^{i, j+k+\ell} \circ\left(1 \otimes m_{w}^{j, k+\ell}\right) \circ\left(1 \otimes 1 \otimes m_{t}^{k, \ell}\right)$ is the composite of a map to $M^{\prime}$ followed by the injection $\iota_{t, w, z}$. We let $u^{\prime}$ be the image of $u_{i} \otimes u_{j} \otimes u_{k} \otimes u_{\ell}$ in $M^{\prime}$.

By Lemma 2.1.4, we can follow the following diagram

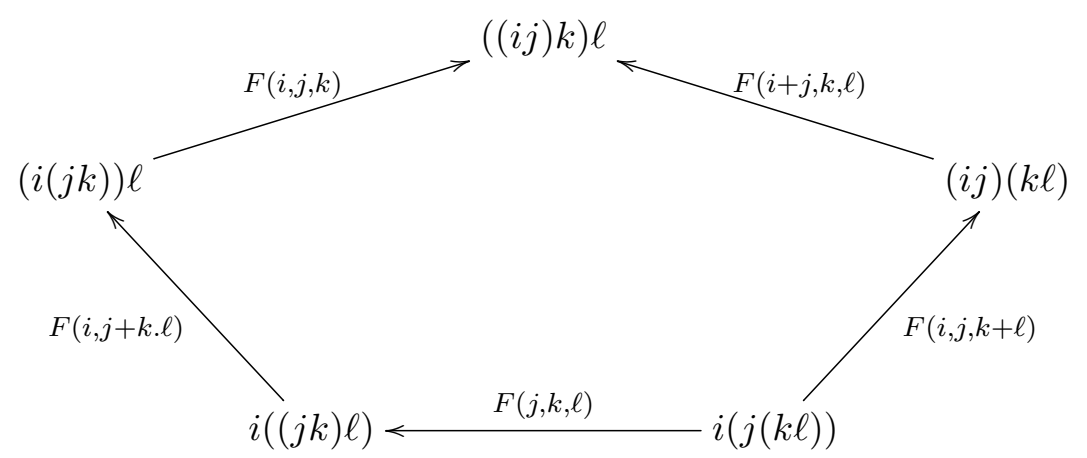


to deduce statements about the composition of other intertwining operators:

(1) The composite $m_{w}^{i+j, k+\ell} \circ\left(1 \otimes m_{t}^{k, \ell}\right) \circ\left(m_{z-w}^{i, j} \otimes 1 \otimes 1\right)$ factors through $M^{\prime}$, and takes $u_{i} \otimes$ $u_{j} \otimes u_{k} \otimes u_{\ell}$ to $F(i, j, k+\ell) u^{\prime}$.

(2) The composite $m_{z}^{i, j+k+\ell} \circ\left(1 \otimes m_{t}^{j+k, \ell}\right) \circ\left(1 \otimes m_{w-t}^{j, k} \otimes 1\right)$ factors through $M^{\prime}$, and takes $u_{i} \otimes u_{j} \otimes u_{k} \otimes u_{\ell}$ to $F(j, k, \ell) u^{\prime}$.

(3) The composite $m_{t}^{i+j+k, \ell} \circ\left(m_{z-t}^{i, j+k} \otimes 1\right) \circ\left(1 \otimes m_{w-t}^{j, k} \otimes 1\right)$ factors through $M^{\prime}$, and takes $u_{i} \otimes u_{j} \otimes u_{k} \otimes u_{\ell}$ to $F(i, j+k, \ell) \cdot F(j, k, \ell) u^{\prime}$.

(4) The composite $m_{t}^{i+j+k, \ell} \circ\left(m_{w-t}^{i+j, k} \otimes 1\right) \circ\left(m_{z-w}^{i, j} \otimes 1 \otimes 1\right)$ factors through $M^{\prime}$, and takes $u_{i} \otimes u_{j} \otimes u_{k} \otimes u_{\ell}$ to both $F(i, j, k) \cdot F(i, j+k, \ell) \cdot F(j, k, \ell) u^{\prime}$ and $F(i+j, k, \ell) \cdot F(i, j, k+\ell) u^{\prime}$.

We find that $F(i, j, k) \cdot F(i, j+k, \ell) \cdot F(j, k, \ell) u^{\prime}=F(i+j, k, \ell) \cdot F(i, j, k+\ell) u^{\prime}$. Since $u^{\prime}$ is a nonzero element of a complex vector space, the result follows.

Definition 2.1.6. Given a one-dimensional $\mathcal{T}$-associativity datum, a choice $\left\{m_{z}^{i, j} \in \mathcal{I}_{i, j}^{i+j}\right\}_{i, j \in A}$ of nonzero intertwining operators is normalized if for all $i \in A, m_{z}^{0, i}=a c t_{z}$ and $m_{z}^{i, 0}=a c t_{z}^{*}$.

Lemma 2.1.7. Suppose we are given a one-dimensional $\mathcal{T}$-associativity datum. Then, for any normalized choice $\left\{m_{z}^{i, j} \in \mathcal{I}_{i, j}^{i+j}\right\}_{i, j \in A}$ of nonzero intertwining operators, we have $F(0, i, j)=F(i, 0, j)=$ $F(i, j, 0)=1$ for all $i, j \in A$. In other words, $F$ describes a normalized group cohomology 3-cocycle in the sense of Definition 1.2.5.

Proof. Fix elements $u \in M_{i}$ and $v \in M_{j}$ such that $m_{w}^{i, j}(u \otimes v) \neq 0$, and set $g(w)=\sum_{n \geq r} g_{n} w^{n}=$ $m_{w}^{i, j}(u \otimes v) \in M_{i+j}((w))$. By the defining property of $F(i, 0, j), m_{z}^{i, j} \circ\left(1 \otimes m_{w}^{0, j}\right)$ and $F(i, 0, j) m_{w}^{i, j} \circ$ $\left(m_{z-w}^{i, 0} \otimes 1\right)$ are given by a single map from $M_{i} \otimes M_{0} \otimes M_{j}$ to $M_{i, j}[[z, w]]\left[z^{-1}, w^{-1},(z-w)^{-1}\right]$, followed by the standard injections. Applying this map to $u \otimes 1 \otimes v$, we find that $m_{z}^{i, j}(u \otimes v)$ and $F(i, 0, j) m_{w}^{i, j}\left(e^{(z-w) L(-1)} u \otimes v\right)$ are expansions of the same element of $M_{i, j}[[z, w]]\left[z^{-1}, w^{-1},(z-w)^{-1}\right]$. The left side is $g(z)$, while the right side is equal to $F(i, 0, j) e^{(z-w) \partial_{w}} g(w)$ by Lemma 1.3.5. By Lemma 1.1.8 applied to $M_{i, j}((w))[[z-w]]$, the right side is also equal to $F(i, 0, j) g(z)$, so $F(i, 0, j)=$ 1.

Now, we use Lemma 2.1.5. By substituting $k=\ell=0$, we obtain $F(i, j, 0) F(i, j, 0) F(j, 0,0)=$ $F(i+j, 0,0) F(i, j, 0)$, and together with the relation $F(j, 0,0)=F(i+j, 0,0)=1$ established in the previous paragraph, we get $F(i, j, 0)=1$. By substituting $i=j=0$, we find that the relation $F(0,0, k) F(0, k, \ell) F(0, k, \ell)=F(0, k, \ell) F(0,0, k+\ell)$ together with the relation $F(0,0, k)=$ $F(0,0, k+\ell)=1$ established in the previous paragraph implies $F(0, k, \ell)=1$.

\subsection{Commutativity.}

Definition 2.2.1. Let $V$ be a $\mathcal{T}$-vertex algebra, let $A$ be an abelian group, and let $\left\{M_{i}\right\}_{i \in A}$ be a set of $V$-modules in $\mathcal{T}$, such that $M_{0}=V$. We define a one-dimensional $\mathcal{T}$-commutativity datum to be a one-dimensional $\mathcal{T}$-associativity datum that satisfies the additional condition that for any $i, j \in A$, and any nonzero $m_{z}^{i, j} \in \mathcal{I}_{i, j}^{i+j}$, the intertwining operator $m_{z}^{i, j, *}$ is an element of $\mathcal{I}_{j, i}^{j+i}$. In other words, for any nonzero $m_{z}^{j, i} \in \mathcal{I}_{j, i}^{j+i}$, there exists $\lambda \in \mathbb{C}^{\times}$such that for any $u \otimes v \in M_{i} \otimes M_{j}$, the elements $\lambda m_{z}^{i, j}(u \otimes v)$ and $e^{z L(-1)} m_{-z}^{j, i}(v \otimes u)$ of $M_{i+j}((z))$ are equal.

Remark 2.2.2. If $\mathcal{T}$-vertex algebras are vertex operator algebras, then a commutativity datum is quite similar to Huang's notion of "ZZZ-graded meromorphic locally grading-restricted conformal intertwining algebra" Huang 2001, where the set $\mathcal{A}$ is our group $A$, and the vector spaces $\mathcal{V}_{i, j}^{k}$ are $\mathcal{I}_{i, j}^{i+j}$ when $k=i+j$ and 0 otherwise. However, Huang requires a convergence condition for all compositions of intertwining operators, while we only require three-fold compositions to be well-behaved. The reader should be aware that although the word "algebra" appears in the 
name, Huang's notion of intertwining algebra does not have a distinguished choice of multiplication operator in the defining data.

Lemma 2.2.3. Suppose we are given a one-dimensional $\mathcal{T}$-commutativity datum. Then for any choice of nonzero intertwining operators $\left\{m_{z}^{i, j} \in \mathcal{I}_{i, j}^{i+j}\right\}_{i, j \in A}$, and any $i, j, k \in A$, the compositions $m_{w}^{i+j, k} \circ\left(m_{z-w}^{i, j} \otimes 1\right)$ and $m_{z}^{i+j, k} \circ\left(m_{w-z}^{j, i} \otimes 1\right) \circ \tau_{(12)}$, factor as maps $M_{i} \otimes M_{j} \otimes M_{k} \rightarrow$ $M_{i+j+k}[[z, w]]\left[z^{-1}, w^{-1},(z-w)^{-1}\right]$ that differ by a constant multiple that does not depend on $k$, followed by inclusions $\iota_{w, z-w}$ and $\iota_{z, z-w}$. In particular, the vector spaces $\tau_{(12)}^{*}\left(\mathcal{I}_{i, j, k}^{i+j+k}\right)$ and $\mathcal{I}_{j, i, k}^{j+i+k}$ are equal.

Proof. Let $u \otimes v \otimes x \in M_{i} \otimes M_{j} \otimes M_{k}$ satisfy $m_{w}^{i+j, k}\left(m_{z-w}^{i, j}(u \otimes v) \otimes x\right) \neq 0 \in M_{i+j+k}((w))((z-w))$. Such an element exists, by the hypothesis that $\mathcal{I}_{i, j, k}^{i+j+k}$ is nonzero. By the condition defining commutativity datum, $\lambda m_{z-w}^{i, j}(u \otimes v)=e^{(z-w) L(-1)} m_{w-z}^{j, i}(v \otimes u)$ for some $\lambda \in \mathbb{C}^{\times}$that depends only on $i$ and $j$. By the translation covariance axiom applied to $m_{w}^{i+j, k}$, we have

$$
m_{w}^{i+j, k}\left(\left(e^{(z-w) L(-1)} m_{w-z}^{j, i}(v \otimes u)\right) \otimes x\right)=e^{(z-w) \partial_{w}} m_{w}^{i+j, k}\left(m_{w-z}^{j, i}(v \otimes u) \otimes x\right)
$$

as elements of $M_{i+j+k}((w))((z-w))$. However, these series are the image of a unique element of $M_{i+j+k}[[z, w]]\left[z^{-1}, w^{-1},(z-w)^{-1}\right]$ along the embedding given in Lemma 1.1.4. Thus, by Lemma 1.1.10, the expression $m_{z}^{i+j, k}\left(m_{w-z}^{j, i}(v \otimes u) \otimes x\right)$ is the expansion in $M_{i+j+k}((z))((z-w))$ of the same element. Assembling these equalities, we find that $\lambda m_{w}^{i+j, k} \circ\left(m_{z-w}^{i, j}(u \otimes v) \otimes x\right)$ and $m_{z}^{i+j, k}\left(m_{w-z}^{j, i}(v \otimes\right.$ $u) \otimes x)$ are expansions of the same nonzero element of $M_{i+j+k}[[z, w]]\left[z^{-1}, w^{-1},(z-w)^{-1}\right]$.

Definition 2.2.4. Given a one dimensional $\mathcal{T}$-commutativity datum, for any choice of nonzero operators $\left\{m_{z}^{i, j} \in \mathcal{I}_{i, j}^{i+j}\right\}_{i, j \in A}$ we define the function $\Omega: A^{\oplus 2} \rightarrow \mathbb{C}^{\times}$by

$$
m_{z}^{i, j}=\Omega(i, j) e^{z L(-1)} m_{-z}^{j, i} \circ \tau_{(12)}
$$

Remark 2.2.5. We note that by Lemma 2.2.3, the following holds for all $k \in A$ :

$$
m_{w}^{i+j, k} \circ\left(m_{z-w}^{i, j} \otimes 1\right)=\Omega(i, j) m_{z}^{j+i, k} \circ\left(m_{-z+w}^{j, i} \otimes 1\right) \circ \tau_{(12)} .
$$

Remark 2.2.6. One may alternatively define $\Omega$ by setting $B(i, j, k)$ to be the defect in the original locality diagram, and setting $\Omega(i, j)=F(i, j, k)^{-1} B(i, j, k) F(j, i, k)$ (see Dong-Lepowsky 1993 Chapter 12). In terms of intertwining operators, we see that the two composite maps

$$
m_{z}^{i, j+k} \circ\left(1 \otimes m_{w}^{j, k}\right) \quad \text { and } \quad F(j, i, k)^{-1} \Omega(i, j) F(i, j, k) m_{w}^{j, i+k} \circ\left(1 \otimes m_{z}^{i, k}\right) \circ \tau_{(12)}
$$

factor through equal maps from $M_{i} \otimes M_{j} \otimes M_{k}$ to $M_{i+j+k}[[z, w]]\left[z^{-1}, w^{-1},(z-w)^{-1}\right]$. Diagrammatically, $\Omega(i, j)$ can be represented as the defect obstructing commutativity in the outer pentagon:

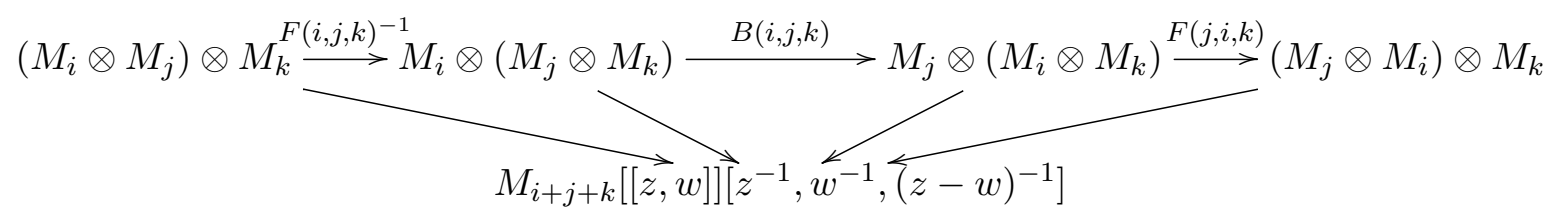

Lemma 2.2.7. Given a one dimensional $\mathcal{T}$-commutativity datum, for any choice of nonzero elements $\left\{m_{z}^{i, j} \in \mathcal{I}_{i, j}^{i+j}\right\}_{i, j \in A}$, the functions $F$ and $\Omega$ satisfy the hexagon conditions:

(1) $F(i, j, k)^{-1} \Omega(i, j+k) F(j, k, i)^{-1}=\Omega(i, j) F(j, i, k)^{-1} \Omega(i, k)$

(2) $F(i, j, k) \Omega(i+j, k) F(k, i, j)=\Omega(j, k) F(i, k, j) \Omega(i, k)$ 
Proof. As in Lemma 2.1.5, for any $i, j, k \in A$, let $u_{i} \in M_{i}, u_{j} \in M_{j}, u_{k} \in M_{k}$ and $u_{0} \in M_{0}=V$ satisfy:

$$
m_{z}^{i, j+k} \circ\left(1 \otimes m_{w}^{j, k}\right) \circ\left(1 \otimes 1 \otimes m_{t}^{k, 0}\right)\left(u_{i} \otimes u_{j} \otimes u_{k} \otimes u_{0}\right) \neq 0 \in M_{i+j+k}((z))((w))((t)) .
$$

For convenience, we write $M^{\prime}=M_{i+j+k}[[z, w, t]]\left[z^{-1}, w^{-1} t^{-1},(z-w)^{-1},(z-t)^{-1},(w-t)^{-1}\right]$. As before, by the existence of $\mathcal{I}_{i, j, k, 0}^{i+j+k}$, a satisfactory choice of elements $\left(u_{i}, u_{j}, u_{k}, u_{0}\right)$ exists, and the map $m_{z}^{i, j+k} \circ\left(1 \otimes m_{w}^{j, k}\right) \circ\left(1 \otimes 1 \otimes m_{t}^{k, 0}\right)$ is the composite of a map to $M^{\prime}$ followed by the injection $\iota_{t, w, z}$. We let $u^{\prime}$ be the image of $u_{i} \otimes u_{j} \otimes u_{k} \otimes u_{0}$ in $M^{\prime}$ in this factorization.

To prove the first equation, we follow the following diagram:

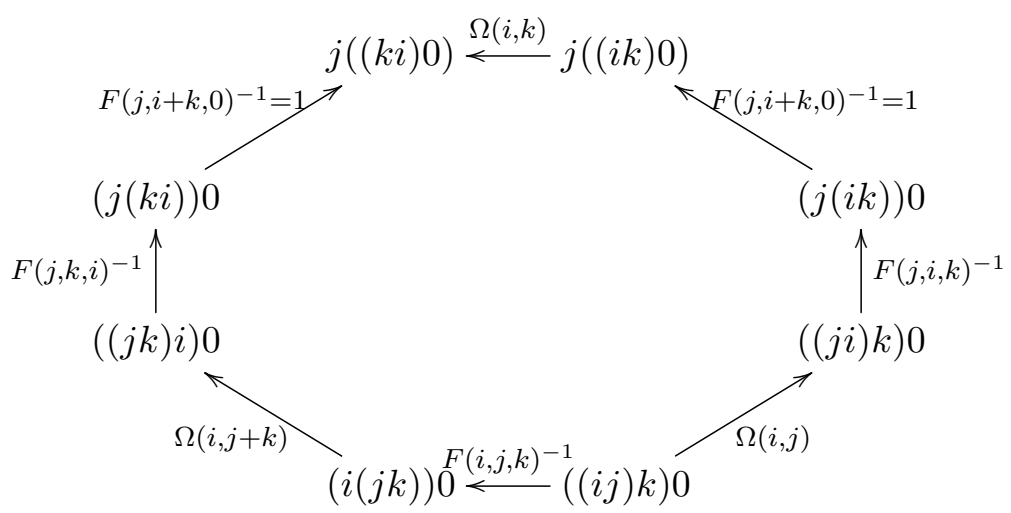

to deduce statements about the composition of the corresponding intertwining operators. The left side of the octagon is traversed as follows:

$((\mathrm{ij}) \mathrm{k}) 0$ : By Lemma 2.1.5, the composite $m_{t}^{i+j+k, 0} \circ\left(m_{w-t}^{i+j, k} \otimes 1\right) \circ\left(m_{z-w}^{i, j} \otimes 1 \otimes 1\right)$ factors through $M^{\prime}$, and takes $u_{i} \otimes u_{j} \otimes u_{k} \otimes u_{0}$ to $F(i, j, k) u^{\prime}$.

$(\mathrm{i}(\mathrm{jk})) 0$ : The composite $m_{t}^{i+j+k, 0} \circ\left(m_{z-t}^{i, j+k} \otimes 1\right) \circ\left(1 \otimes m_{w-t}^{j, k} \otimes 1\right)$ factors through $M^{\prime}$, and takes $u_{i} \otimes u_{j} \otimes u_{k} \otimes u_{0}$ to $u^{\prime}$.

((jk)i)0: By Lemma 2.2.3, the composite $m_{z}^{j+k+i, 0} \circ\left(m_{t-z}^{j+k, i} \otimes 1\right) \circ\left(m_{w-t}^{j, k} \otimes 1 \otimes 1\right)$ factors through $M^{\prime}$ and takes $u_{i} \otimes u_{j} \otimes u_{k} \otimes u_{0}$ to $\Omega(i, j+k) u^{\prime}$.

(j(ki))0: The composite $m_{t}^{j+k+i, 0} \circ\left(m_{w-z}^{j, k+i} \otimes 1\right) \circ\left(1 \otimes m_{t-z}^{k, i} \otimes 1\right)$ factors through $M^{\prime}$, and takes $u_{i} \otimes u_{j} \otimes u_{k} \otimes u_{0}$ to $F(j, k, i)^{-1} \Omega(i, j+k) u^{\prime}$.

$\mathrm{j}((\mathrm{ki}) 0)$ : By Lemma 2.1.7 the composite $m_{w-z}^{j, k+i} \circ\left(1 \otimes m_{z}^{k+i, 0}\right) \circ\left(1 \otimes m_{t-z}^{k, i} \otimes 1\right)$ factors through $M^{\prime}$, and takes $u_{i} \otimes u_{j} \otimes u_{k} \otimes u_{0}$ to $F(j, i+k, 0)^{-1} F(j, k, i)^{-1} \Omega(i, j+k) u^{\prime}=F(j, k, i)^{-1} \Omega(i, j+k) u^{\prime}$.

The right side of the octagon is traversed as follows:

$((\mathrm{ji}) \mathrm{k}) 0$ : The composite $m_{t}^{j+i+k, 0} \circ\left(m_{z-t}^{j+i, k} \otimes 1\right) \circ\left(m_{w-z}^{j, i} \otimes 1 \otimes 1\right)$ factors through $M^{\prime}$, and takes $u_{i} \otimes u_{j} \otimes u_{k} \otimes u_{0}$ to $\Omega(i, j) F(i, j, k) u^{\prime}$.

$(\mathrm{j}(\mathrm{ik})) 0$ : The composite $m_{t}^{j+i+k, 0} \circ\left(m_{w-t}^{j, i+k} \otimes 1\right) \circ\left(1 \otimes m_{z-t}^{i, k} \otimes 1\right)$ factors through $M^{\prime}$, and takes $u_{i} \otimes u_{j} \otimes u_{k} \otimes u_{0}$ to $F(j, i, k)^{-1} \Omega(i, j) F(i, j, k) u^{\prime}$.

$\mathrm{j}((\mathrm{ik}) 0)$ : The composite $m_{w-t}^{j, i+k} \circ\left(1 \otimes m_{t}^{i+k, 0}\right) \circ\left(1 \otimes m_{z-t}^{i, k} \otimes 1\right)$ factors through $M^{\prime}$, and takes $u_{i} \otimes$ $u_{j} \otimes u_{k} \otimes u_{0}$ to $F(j, i+k, 0)^{-1} F(j, i, k)^{-1} \Omega(i, j) F(i, j, k) u^{\prime}=F(j, i, k)^{-1} \Omega(i, j) F(i, j, k) u^{\prime}$.

$\mathrm{j}((\mathrm{ki}) 0)$ : The composite $m_{w-z}^{j, k+i} \circ\left(1 \otimes m_{z}^{k+i, 0}\right) \circ\left(1 \otimes m_{t-z}^{k, i} \otimes 1\right)$ factors through $M^{\prime}$, and takes $u_{i} \otimes$ $u_{j} \otimes u_{k} \otimes u_{0}$ to $\Omega(i, k) F(j, i, k)^{-1} \Omega(i, j) F(i, j, k) u^{\prime}$

We see that $F(j, k, i)^{-1} \Omega(i, j+k) u^{\prime}=\Omega(i, k) F(j, i, k)^{-1} \Omega(i, j) F(i, j, k) u^{\prime}$, and because $u^{\prime}$ is a nonzero element of a complex vector space, the first hexagon equation holds. 
To prove the second equation, we follow the following diagram:

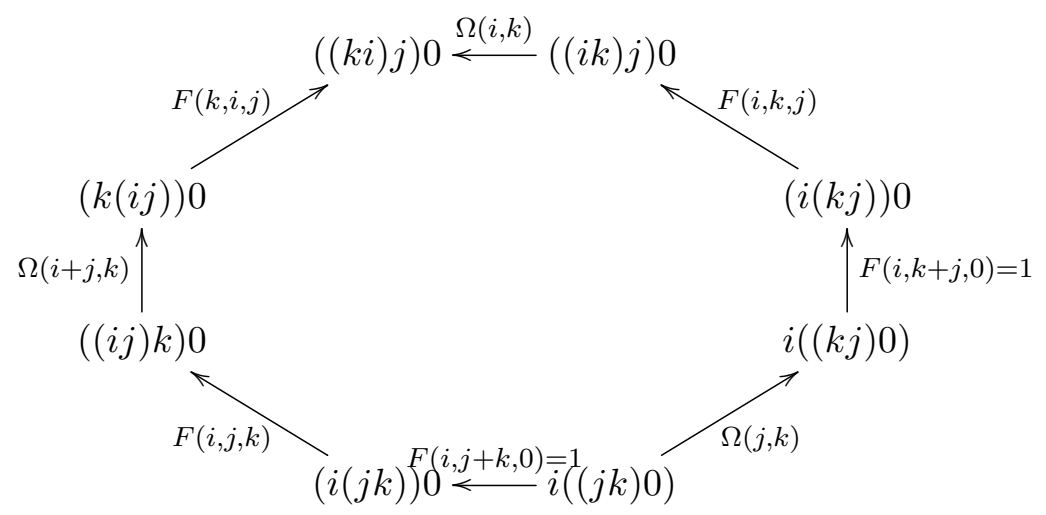

to deduce statements about the composition of the corresponding intertwining operators. After omitting previous evaluations, the left side of the octagon is traversed as follows:

$((\mathrm{ij}) \mathrm{k}) 0$ : The composite $m_{t}^{i+j+k, 0} \circ\left(m_{w-t}^{i+j, k} \otimes 1\right) \circ\left(m_{z-w}^{i, j} \otimes 1 \otimes 1\right)$ factors through $M^{\prime}$ and takes $u_{i} \otimes u_{j} \otimes u_{k} \otimes u_{0}$ to $F(i, j, k) u^{\prime}$.

$(\mathrm{k}(\mathrm{ij})) 0$ : The composite $m_{w}^{k+i+j, 0} \circ\left(m_{t-w}^{k, i+j} \otimes 1\right) \circ\left(1 \otimes m_{z-w}^{i, j} \otimes 1\right)$ factors through $M^{\prime}$, and takes $u_{i} \otimes u_{j} \otimes u_{k} \otimes u_{0}$ to $\Omega(i+j, k) F(i, j, k) u^{\prime}$.

$((\mathrm{ki}) \mathrm{j}) 0$ : The composite $m_{w}^{k+i+j, 0} \circ\left(m_{z-w}^{k+i, j} \otimes 1\right) \circ\left(m_{t-z}^{k, i} \otimes 1 \otimes 1\right)$ factors through $M^{\prime}$ and takes $u_{i} \otimes u_{j} \otimes u_{k} \otimes u_{0}$ to $F(k, i, j) \Omega(i+j, k) F(i, j, k) u^{\prime}$.

The right side of the octagon is traversed as follows:

$\mathrm{i}((\mathrm{kj}) 0)$ : The composite $m_{z-w}^{i, k+j} \circ\left(1 \otimes m_{w}^{k+j, 0}\right) \circ\left(1 \otimes m_{t-w}^{k, j} \otimes 1\right)$ factors through $M^{\prime}$, and takes $u_{i} \otimes$ $u_{j} \otimes u_{k} \otimes u_{0}$ to $\Omega(j, k) u^{\prime}$.

$(\mathrm{i}(\mathrm{kj})) 0$ : The composite $m_{w}^{i+k+j, 0} \circ\left(m_{z-w}^{i, k+j} \otimes 1\right) \circ\left(1 \otimes m_{t-w}^{k, j} \otimes 1\right)$ factors through $M^{\prime}$, and takes $u_{i} \otimes u_{j} \otimes u_{k} \otimes u_{0}$ to $F(i, k+j, 0) \Omega(j, k) u^{\prime}=\Omega(j, k) u^{\prime}$.

$((\mathrm{ik}) \mathrm{j}) 0$ : The composite $m_{w}^{i+k+j, 0} \circ\left(m_{t-w}^{i+k, j} \otimes 1\right) \circ\left(m_{z-t}^{i, k} \otimes 1 \otimes 1\right)$ factors through $M^{\prime}$ and takes $u_{i} \otimes u_{j} \otimes u_{k} \otimes u_{0}$ to $F(i, k, j) \Omega(j, k) u^{\prime}$.

$((\mathrm{ki}) \mathrm{j}) 0$ : The composite $m_{w}^{k+i+j, 0} \circ\left(m_{z-w}^{k+i, j} \otimes 1\right) \circ\left(m_{t-z}^{k, i} \otimes 1 \otimes 1\right)$ factors through $M^{\prime}$ and takes $u_{i} \otimes u_{j} \otimes u_{k} \otimes u_{0}$ to $\Omega(i, k) F(i, k, j) \Omega(j, k) u^{\prime}$.

We see that $F(k, i, j) \Omega(i+j, k) F(i, j, k) u^{\prime}=\Omega(i, k) F(i, k, j) \Omega(j, k) u^{\prime}$, and because $u^{\prime}$ is a nonzero element of a complex vector space, the second hexagon equation holds.

Proposition 2.2.8. Let $V$ be a $\mathcal{T}$-vertex algebra, let $A$ be an abelian group, and let $\left\{M_{i}\right\}_{i \in A}$ be a set of $V$-modules in $\mathcal{T}$, such that $M_{0}=V$. Given a one dimensional $\mathcal{T}$-commutativity datum, and any normalized choice of nonzero elements $\left\{m_{z}^{i, j} \in \mathcal{I}_{i, j}^{i+j}\right\}_{i, j \in A}$, the pair $(F, \Omega)$ derived from $\left\{m^{i, j}\right\}$ forms a normalized abelian 3-cocycle for $A$ with coefficients in $\mathbb{C}^{\times}$.

Proof. This follows from the fact that the abelian 3-cocycle condition is given by the pentagon condition (proved in Lemma 2.1.5), and the hexagon condition (proved in Lemma 2.2.7).

Remark 2.2.9. Before we can state our main obstruction results, we have to deal with the possibility of finiteness conditions obstructing the formation of $\mathcal{T}$-vertex algebras. In particular, vertex operator algebras satisfy the condition that eigenvalues of $L(0)$ have finite multiplicity, and this may not be satisfied when $A$ is infinite. The standard example is given by vertex algebras of indefinite lattices, such as the fake monster vertex algebra (introduced in Borcherds 1986] without a name), which are built from modules of a Heisenberg vertex operator algebra, but are not vertex operator algebras because of this spectral condition.

Definition 2.2.10. We say that a collection of modules in $\mathcal{T}$ is $\mathcal{T}$-compatible if their direct sum is a module in $\mathcal{T}$. 
Proposition 2.2.11. Let $V$ be a $\mathcal{T}$-vertex algebra, let $A$ be an abelian group, and let $\left\{M_{i}\right\}_{i \in A}$ be a $\mathcal{T}$-compatible set of $V$-modules in $\mathcal{T}$, such that $M_{0}=V$. Suppose we are given a one dimensional $\mathcal{T}$-commutativity datum, and a normalized choice of nonzero elements $\left\{m_{z}^{i, j} \in \mathcal{I}_{i, j}^{i+j}\right\}_{i, j \in A}$. Then we define a vector space $W=\bigoplus_{i \in A} M_{i}$, a derivation $L(-1)^{W}: W \rightarrow W$ by its restriction to each $M_{i}$, and a multiplication map $m_{z}^{W}: W \otimes W \rightarrow W((z))$ as the sum of its restrictions $m_{z}^{i, j}$. The tuple $\left(W, \mathbf{1}_{V}, L(-1)^{W}, m_{z}^{W}\right)$ is a $\mathcal{T}$-vertex algebra if and only if the abelian 3-cocycle $(F, \Omega)$ is identically 1 .

Proof. Since $F$ measures the failure of associativity, the associativity property holds if and only if $F$ is identically $1 . \Omega$ is identically 1 if and only if skew-symmetry holds. Since locality is equivalent to the combination of associativity and skew-symmetry, we obtain a vertex algebra. We now need to show that we obtain a $\mathcal{T}$-vertex algebra for the various values of $\mathcal{T}$ under consideration.

(1) The unrestricted setting: We are already done.

(2) Worldsheet symmetry: If $V$ is weighted (resp. Möbius, quasi-conformal), our modules come with a collection of operators $\{L(i)\}$, and the compatibility condition for module structures and intertwining operators is equivalent for the vertex algebra to have the new worldsheet symmetry. If $V$ is conformal, the conformal condition on modules implies the conformal vector induces a Virasoro action on $W$.

(3) VOAs: Our assumption that $\left\{M_{i}\right\}_{i \in A}$ is $\mathcal{T}$-compatible implies $W$ has spectrum bounded below and finite dimensional eigenspaces.

(4) $G$-equivariant versions: The compatibility condition implies $g m_{z}^{i, j}\left(u_{i} \otimes u_{j}\right)=m_{z}^{i, j}\left(g u_{i} \otimes g u_{j}\right)$ for all $u_{i} \in M_{i}$ and $u_{j} \in M_{j}$. This is precisely the condition for $g$ to be a vertex algebra automorphism of $W$. For the action of $G$ to respect additional worldsheet symmetry on $W$, it is necessary and sufficient that $g L(k)=L(k) g$ for all relevant $k$, and this one of the conditions for a $G$-action on $M_{i}$ to be compatible with the $V$-module structure.

Definition 2.2.12. Let $V$ be a $\mathcal{T}$-vertex algebra, let $A$ be an abelian group, and let $\left\{M_{i}\right\}_{i \in A}$ be a set of $V$-modules in $\mathcal{T}$, such that $M_{0}=V$. Suppose we are given spaces $\mathcal{I}_{i, j}^{i+j}$ of $\mathcal{T}$-intertwining operators $\left\{m_{z}^{i, j}: M_{i} \otimes M_{j} \rightarrow M_{i+j}((z))\right\}_{i, j \in A}$. We define a " $\lambda$-twist" action of the abelian 2cochain group $C_{a b}^{2}\left(A, \mathbb{C}^{\times}\right)$on $\bigoplus \mathcal{I}_{i, j}^{i+j}$ by sending any cochain $(i, j) \mapsto \lambda_{i, j} \in \mathbb{C}^{\times}$to the rescaling $\operatorname{map}\left\{m^{i, j}(z)\right\} \mapsto\left\{\lambda_{i, j} m^{i, j}(z)\right\}$.

Theorem 2.2.13. Let $V$ be a $\mathcal{T}$-vertex algebra, let $A$ be an abelian group, and let $\left\{M_{i}\right\}_{i \in A}$ be a set of $V$-modules in $\mathcal{T}$, such that $M_{0}=V$. Given a one dimensional $\mathcal{T}$-commutativity datum attached to $V$ and $\left\{M_{i}\right\}_{i \in A}$, and any normalized choice of nonzero elements $\left\{m_{z}^{i, j} \in \mathcal{I}_{i, j}^{i+j}\right\}_{i, j \in A}$, the following hold:

(1) The action of $C_{a b}^{2}\left(A, \mathbb{C}^{\times}\right)$on the total intertwining operator space $\bigoplus \mathcal{I}_{i, j}^{i+j}$ given in Definition 2.2.12 induces the canonical translation action $\left(\left\{\lambda_{i, j}\right\},(F, \Omega)\right) \mapsto(d \lambda \cdot(F, \Omega))$ on the group of abelian 3-cocycles by coboundaries. This action is transitive on representatives of any fixed cohomology class in $H_{a b}^{3}\left(A, \mathbb{C}^{\times}\right)$, with stabilizer given by the group of abelian 2-cocycles $\left\{\lambda_{i, j}\right\}$.

(2) The function $A \rightarrow \pm 1$ defined by $i \mapsto \Omega(i, i)$ is a quadratic form invariant under $\lambda$-twist by 2-cochains. In particular, the abelian cohomology class of $(F, \Omega)$ is canonically attached to the commutativity datum.

(3) If $\left\{M_{i}\right\}$ is $\mathcal{T}$-compatible, then the quadratic form $i \mapsto \Omega(i, i)$ is identically one if and only if there exists a normalized 2-cochain $\lambda$ such that $\left\{\lambda_{i, j} m_{z}^{i, j}\right\}$ describe an A-graded $\mathcal{T}$-vertex algebra structure on $\bigoplus_{i \in A} M_{i}$.

(4) The A-graded $\mathcal{T}$-vertex algebra structure on $\bigoplus_{i \in A} M_{i}$ is unique up to isomorphism if it exists. 
Proof. For the first claim, it suffices to verify that the cocycle $(F, \Omega)$ is shifted by the abelian differential of $\lambda$. This is essentially the content of Dong-Lepowsky 1993 Remark 12.23 (for finite order cocycles), and Bakalov-Kac 2006] section 3.4 (for the case of trivial $F$ ), and the arguments given in the literature extend without substantial change.

In the second statement, the invariance of the abelian cohomology class follows from the fact that normalized 2-cochains act simply transitively on the set of normalized choices of intertwining operators. Both the fact that $i \rightarrow \Omega(i, i)$ is quadratic and the fact that it is invariant follow from Lemma 1.2 .3 .

For the third statement, Proposition 2.2.11 implies it is necessary and sufficient that we obtain a trivial abelian 3-cocycle. By Lemma 1.2.6, the abelian differential defines a transitive action of normalized 2-cochains on the normalized abelian 3-cocycles in the abelian cohomology class of $(F, \Omega)$. In other words there exists such $\lambda$ if and only if the pair $(F, \Omega)$ is an abelian 3-cocycle in the zero cohomology class. By Lemma 1.2 .3 , the abelian cohomology class is determined by its trace, and is trivial if and only if $\Omega(i, i)=1$ for all $i$.

For the last claim, suppose a $\mathcal{T}$-vertex algebra compatible with the commutativity datum exists. Then a $\lambda$-twist yields a $\mathcal{T}$-vertex algebra precisely when $\lambda$ is an abelian 2-cocycle, i.e., we have an action of $Z_{a b}^{2}\left(A, \mathbb{C}^{\times}\right)=Z^{3}\left(K(A, 2), \mathbb{C}^{\times}\right)$on the set of isomorphism classes of $A$-graded $\mathcal{T}$ vertex algebras compatible with the commutativity datum. Since $\lambda$-twisting is transitive on the set of choices of multiplication rules, the cocycle group acts transitively on the set of isomorphism classes. Furthermore, this action factors through $H_{a b}^{2}\left(A, \mathbb{C}^{\times}\right)$, because twists by 2-coboundaries induce isomorphisms by abelian 1-cochains given by multiples of identity $c_{i} \cdot I d: M_{i} \rightarrow M_{i}$. By Lemma 1.2.4 $H_{a b}^{2}\left(A, \mathbb{C}^{\times}\right)$is trivial. Thus, the set of isomorphism classes has a transitive action of a trivial group, and hence has one element.

Remark 2.2.14. The uniqueness claim is similar to the argument in the proof of Proposition 5.3 in Dong-Mason 2002. However, the argument there needs some minor repair: all instances of "2-cocycle" and "two cocycle" should be changed to "abelian 2-cocycle". Indeed, $H^{2}\left(A, \mathbb{C}^{\times}\right)$is nontrivial for any non-cyclic finite abelian group $A$.

2.3. Evenness. The condition that $\Omega(i, i)=1$ for all $i \in A$ that appears in the third claim of Theorem 2.2.13 is not automatically satisfied. Indeed, any supercommutative $\operatorname{ring} R$ whose odd part has nonzero three-fold products can be written as follows: We set $A=\mathbb{Z} / 2 \mathbb{Z}, V=M_{0}=R_{\text {even }}$, $M_{1}=R_{o d d}, L(-1)$ is the zero map, and $m^{i, j}$ are the restrictions of multiplication on $R$. We find that the spans of $\left\{m^{i, j}\right\}$ form a one-dimensional $\mathcal{T}$-commutativity datum, but the abelian 3-cocycle is nontrivial: $F$ is the zero map, but $\Omega(i, j)=(-1)^{i j}$. This counterexample works in the weighted, Möbius, quasi-conformal, and conformal cases, because we may define a conformal element by setting $\omega=0$, causing $L(i)$ to act by zero for all $i \in \mathbb{Z}$. We explore this condition further here.

Definition 2.3.1. Let $V$ be a $\mathcal{T}$-vertex algebra, and let $M_{1}$ and $M_{2}$ be $V$-modules in $\mathcal{T}$. A $\mathcal{T}$ intertwining operator $I_{z}: M_{1} \otimes M_{1} \rightarrow M_{2}((z))$ is called even if one of the following conditions holds:

(1) For any $u \in M_{1}, I_{z}(u \otimes u)=0$, and for any $u, v \in M_{1}$ such that $I_{z}(u \otimes v) \neq 0$, we have $I_{z}(u \otimes v) \in z^{n} M_{2}[[z]] \backslash z^{n+1} M_{2}[[z]]$, where $n$ is odd.

(2) There exists $u \in M_{1}$ such that $I_{z}(u \otimes u) \in z^{n} M_{2}[[z]] \backslash z^{n+1} M_{2}[[z]]$, where $n$ is even.

$I_{z}$ is called odd if one of the following conditions holds:

(1) For any $u \in M_{1}, I_{z}(u \otimes u)=0$, and for any $u, v \in M_{1}$ such that $I_{z}(u \otimes v) \neq 0$, we have $I_{z}(u \otimes v) \in z^{n} M_{2}[[z]] \backslash z^{n+1} M_{2}[[z]]$, where $n$ is even.

(2) There exists $u \in M_{1}$ such that $I_{z}(u \otimes u) \in z^{n} M_{2}[[z]] \backslash z^{n+1} M_{2}[[z]]$, where $n$ is odd.

A commutativity datum is even if for all $i \in A$, any nonzero $m_{z}^{i, i} \in \mathcal{I}_{i, i}^{2 i}$ is even.

Remark 2.3.2. By Proposition 11.9 in Dong-Lepowsky 1993, if $M_{1}$ is irreducible, then $I_{z}(u \otimes u) \neq 0$ for any nonzero $u \in M_{1}$ and any nonzero intertwining operator $I_{z}: M_{1} \otimes M_{1} \rightarrow M_{2}((z))$. In this 
case, the term "evenness" is reasonable, since it refers to the exponent of the lowest order nonzero term in $I_{z}(u \otimes u)$.

Lemma 2.3.3. Let $V$ be a $\mathcal{T}$-vertex algebra, let $M_{1}$ and $M_{2}$ be $V$-modules in $\mathcal{T}$, and let $I_{z}$ : $M_{1} \otimes M_{1} \rightarrow M_{2}((z))$ be a nonzero $\mathcal{T}$-intertwining operator. If $I_{z}$ is proportional to $I_{z}^{*}$, then $I_{z}$ is either even or odd, and rescaling an intertwining operator does not change evenness or oddness. In particular, if we are given a one dimensional $\mathcal{T}$-commutativity datum, for any $i \in A$, any nonzero $m^{i, i} \in \mathcal{I}_{i, i}^{2 i}$ is either even or odd. Furthermore, for all $i \in A, m_{z}^{i, i}$ is even if and only if $\Omega(i, i)=1$, and $m_{z}^{i, i}$ is odd if and only if $\Omega(i, i)=-1$. In particular, the commutativity datum is even if and only if $\Omega(i, i)=1$ for all $i \in A$.

Proof. We apply the technique used in the proof of Lemma 1.4.7.

Alternating case: If $I_{z}(u \otimes u)=0$ for all $u \in M_{1}$, then bilinearity implies that for any $u, v \in M_{1}$, $I_{z}(u \otimes v)=-I_{z}(v \otimes u)$. Since $I_{z}$ is nonzero, there exist $u, v \in M_{1}$ such that $I_{z}(u \otimes v) \in z^{n} M_{2}[[z]] \backslash$ $z^{n+1} M_{2}[[z]]$ for some integer $n$, so we write $I_{z}(u \otimes v)=g_{n} z^{n}+g_{n+1} z^{n+1}+\cdots$ with $g_{n}$ nonzero. Then by our assumption about $I_{z}^{*}$ :

$$
\begin{aligned}
g_{n} z^{n}+\cdots & =I_{z}(u \otimes v) \\
& =\Omega(1,1) e^{z L(-1)} I_{-z}^{*}(v \otimes u) \\
& =-\Omega(1,1) e^{z L(-1)} g_{n}(-z)^{n}+\cdots \\
& =(-1)^{n+1} \Omega(1,1) g_{n} z^{n}+\cdots
\end{aligned}
$$

If $n$ is odd (i.e., if $I_{z}$ is even), then $g_{n}=\Omega(1,1) g_{n}$, and $\Omega(1,1)=1$. If $n$ is even (i.e., if $I_{z}$ is odd), then $g_{n}=-\Omega(1,1) g_{n}$, and $\Omega(1,1)=-1$.

Non-alternating case: If there exists $u \in M_{1}$ such that $I_{z}(u \otimes u) \neq 0$, then $I_{z}(u \otimes u) \in z^{n} M_{2}[[z]] \backslash$ $z^{n+1} M_{2}[[z]]$ for some integer $n$. We write $I_{z}(u \otimes u)=g_{n} z^{n}+g_{n+1} z^{n+1}+\cdots$ with $g_{n}$ nonzero. Then by our assumption about $I_{z}^{*}$ :

$$
\begin{aligned}
g_{n} z^{n}+\cdots & =I_{z}(u \otimes u) \\
& =\Omega(1,1) e^{z L(-1)} I_{-z}^{*}(u \otimes u) \\
& =\Omega(1,1) e^{z L(-1)} g_{n}(-z)^{n}+\cdots \\
& =(-1)^{n} \Omega(1,1) g_{n} z^{n}+\cdots
\end{aligned}
$$

If $n$ (hence $I_{z}$ ) is even, then $g_{n}=\Omega(1,1) g_{n}$, and $\Omega(1,1)=1$. If $n$ (hence $I_{z}$ ) is odd, then $g_{n}=-\Omega(1,1) g_{n}$, and $\Omega(1,1)=-1$.

The corresponding statements for commutativity data follow in a straightforward way.

Lemma 2.3.4. Let $V^{\prime} \subset V$ be an embedding of vertex algebras, let $M_{1}$ and $M_{2}$ be $V$-modules in $\mathcal{T}$, and let $I_{z}: M_{1} \otimes M_{1} \rightarrow M_{2}((z))$ be an intertwining operator in $\mathcal{T}$ proportional to $I_{z}^{*}$. If there is a $V^{\prime}$-submodule $M_{1}^{\prime} \subset M_{1}$ in $\mathcal{T}$ such that the restriction of $I_{z}$ to $M_{1}^{\prime} \otimes M_{1}^{\prime}$ is nonzero, then $I_{z}$ is even if and only if its restriction is.

Proof. By Lemma 2.3.3, $I_{z}$ is either even or odd, and the same is true of the restriction. Then evenness can be tested on a single pair of vectors.

Lemma 2.3.5. Let $V$ and $V^{\prime}$ be $\mathcal{T}$-vertex algebras, let $I_{z}: M_{1} \otimes M_{1} \rightarrow M_{2}\left((z)\right.$ ) (resp. $I_{z}^{\prime}: M_{1}^{\prime} \otimes$ $\left.M_{1}^{\prime} \rightarrow M_{2}^{\prime}((z))\right)$ be a $\mathcal{T}$-intertwining operator of $V$-modules (resp. $V^{\prime}$-modules) that is proportional to $I_{z}^{*}$ (resp. $\left.I_{z}^{\prime, *}\right)$. Then $I_{z} \otimes I_{z}^{\prime}:\left(M_{1} \otimes M_{1}^{\prime}\right) \otimes\left(M_{1} \otimes M_{1}^{\prime}\right) \rightarrow\left(M_{2} \otimes M_{2}^{\prime}\right)((z))$ is even if and only if either $I_{z}$ and $I_{z}^{\prime}$ are both even or both odd. If exactly one of $I_{z}$ and $I_{z}^{\prime}$ is even, then $I_{z} \otimes I_{z}^{\prime}$ is odd.

Proof. This follows straightforwardly from the definition. 
Proposition 2.3.6. Let $V$ be a $\mathcal{T}$-vertex algebra, let $A$ be an abelian group, and let $\left\{M_{i}\right\}_{i \in A}$ be a $\mathcal{T}$-compatible set of $V$-modules in $\mathcal{T}$, such that $M_{0}=V$. Given a one dimensional even $\mathcal{T}$ commutativity datum, there exists a choice of nonzero elements $\left\{m_{z}^{i, j} \in \mathcal{I}_{i, j}^{i+j}\right\}_{i, j \in A}$ that defines a $\mathcal{T}$-vertex algebra structure on $\bigoplus_{i \in A} M_{i}$. Furthermore, the vertex algebra structure is unique up to isomorphism.

Proof. By Theorem 2.2.13, to prove the first assertion, it suffices to show that $\Omega(i, i)=1$ for all $i \in A$. This follows from Lemma 2.3.3, because the commutativity datum is even. The uniqueness follows from the last claim in Theorem 2.2.13.

Corollary 2.3.7. Let $V$ be a $\mathcal{T}$-vertex algebra, let $A$ be an abelian group, let $2 \cdot A$ denote the subgroup of $A$ whose elements are even multiples, and let $\left\{M_{i}\right\}_{i \in A}$ be a set of $V$-modules in $\mathcal{T}$, such that $M_{0}=V$, and $\left\{M_{i}\right\}_{i \in 2 A}$ is $\mathcal{T}$-compatible. Given a one dimensional $\mathcal{T}$-commutativity datum, there exists a choice of nonzero elements $\left\{m_{z}^{i, j} \in \mathcal{I}_{i, j}^{i+j}\right\}_{i, j \in 2 \cdot A}$ that defines a $\mathcal{T}$-vertex algebra structure on $\bigoplus_{i \in 2 \cdot A} M_{i}$. In particular, evenness is automatic for 2-divisible groups, such as finite abelian groups of odd order.

Proof. By 2.3.3, the function $i \mapsto \Omega(i, i)$ takes values in \pm 1 , and by Lemma 1.2 .3 , it is a quadratic form, so by Lemma $1.2 .8, i \mapsto \Omega(2 i, 2 i)$ is identically 1 . Then the result follows from Proposition 2.3 .6 .

Corollary 2.3.8. Given a $\mathcal{T}$-commutativity datum for a $\mathcal{T}$-compatible set of modules $\left\{M_{i}\right\}_{i \in A}$, the sum $\bigoplus_{i \in A} M_{i}$ admits a vertex algebra structure induced by the intertwining operators if and only if for each coset $2 A+k$ in $A$, there exists some representative $i \in 2 A+k$ and an even intertwining operator $m_{z}^{i, i} \in \mathcal{I}_{i, i}^{2 i}$.

Proof. By Lemma 1.2.8, any \pm 1 -valued quadratic form on $A$ is identically 1 on $2 A$, and constant on $2 A+k$. Thus, evenness of one intertwining operator is equivalent to evenness of the commutativity datum.

2.4. Modules and intertwining operators. In addition to building vertex algebras from parts, we would like to build new modules and consider intertwining operators between them.

Definition 2.4.1. Let $V$ be a $\mathcal{T}$-vertex algebra, let $A$ be an abelian group, let $\left\{\mathcal{I}_{i, j}^{i+j}\right\}_{i, j \in A}$ be an even $\mathcal{T}$-commutativity datum with modules $\left\{M_{i}\right\}_{i \in A}$, let $S$ be a set equipped with an $A$-action (written as addition), and let $\left\{M_{s}\right\}_{s \in S}$ be a set of $V$-modules in $\mathcal{T}$. We define a $\mathcal{T}$-module datum to be a set $\left\{\mathcal{I}_{i, s}^{i+s}\right\}_{i \in A, s \in S}$ of one-dimensional vector spaces, whose elements are $\mathcal{T}$-intertwining operators $M_{i} \otimes M_{s} \rightarrow M_{i+s}((z))$, such that:

(1) For each $i, j \in A, s \in S$, there exists a one dimensional vector space $\mathcal{I}_{i, j, s}^{i+j+s}$ whose elements are maps $M_{i} \otimes M_{j} \otimes M_{s} \rightarrow M_{i+j+s}[[w, z]]\left[w^{-1}, z^{-1},(w-z)^{-1}\right]$, such that composition of $\mathcal{T}$ intertwining operators, combined with the canonical injections from Lemma 1.1.4, induces isomorphisms from $\mathcal{I}_{i, j+s}^{i+j+s} \otimes \mathcal{I}_{j, s}^{j+s}$ to $\mathcal{I}_{i, j, s}^{i+j+s}$ and from $\mathcal{I}_{i+j, s}^{i+j+s} \otimes \mathcal{I}_{i, j}^{i+j}$ to $\mathcal{I}_{i, j, s}^{i+j+s}$.

(2) For any $i, j, k \in A, s \in S$, there exists a one-dimensional space $\mathcal{I}_{i, j, k, s}^{i+j+k+s}$ whose elements are maps:

$$
M_{i} \otimes M_{j} \otimes M_{k} \otimes M_{s} \rightarrow M_{i+j+k+s}[[z, w, t]]\left[z^{-1}, w^{-1} t^{-1},(z-w)^{-1},(z-t)^{-1},(w-t)^{-1}\right]
$$

such that composition of intertwining operators, combined with one of the canonical injections from Lemma 1.1.6, induces an isomorphism from $\mathcal{I}_{i, j+k+s}^{i+j+k+s} \otimes \mathcal{I}_{j, k+s}^{j+k+s} \otimes \mathcal{I}_{k, s}^{k+s}$ to $\mathcal{I}_{i, j, k, s}^{i+j+k+s}$.

(3) The space $\mathcal{I}_{0, s}^{s}$ is spanned by the module structure map act $t_{z}^{s}: V \otimes M_{s} \rightarrow M_{s}((z))$. 
Suppose we are given a choice of nonzero intertwining operators $\left\{m_{z}^{i, j}\right\}$ inducing a $\mathcal{T}$-vertex algebra structure on $\bigoplus_{i \in A} M_{i}$. Given a $\mathcal{T}$-module datum, and any choice of nonzero intertwining operators $\left\{m_{z}^{i, s} \in \mathcal{I}_{i, s}^{i+s}\right\}_{i \in A, s \in S}$, we define the function $\Phi: A^{\oplus 2} \times S \rightarrow \mathbb{C}^{\times}$by

$$
m_{z}^{i, j+s} \circ\left(1 \otimes m_{w}^{j, s}\right)=\Phi(i, j, s) m_{w}^{i+j, s} \circ\left(m_{z-w}^{i, j} \otimes 1\right)
$$

Lemma 2.4.2. Given a $\mathcal{T}$-module datum, and any choice of nonzero intertwining operators $\left\{m_{z}^{i, s} \in\right.$ $\left.\mathcal{I}_{i, s}^{i+s}\right\}_{i \in A, s \in S}$, the function $\Phi$ satisfies the identity:

$$
\Phi(i, j+k, s) \Phi(j, k, s)=\Phi(i+j, k, s) \Phi(i, j, k+s)
$$

for any $i, j, k \in A, s \in S$. In other words, $\Phi$ is a 2-cocycle for $A$ with coefficients in the A-module Fun $\left(S, \mathbb{C}^{\times}\right)$. The group of 1 -cochains $\left\{\lambda_{i, s}\right\}_{i \in A, s \in S}$ (which can be viewed as maps $A \times S \rightarrow \mathbb{C}^{\times}$) acts by rescaling intertwining operators, and it acts transitively on the set of 2-cocycles in the same cohomology class, by coboundary translation. The 2-cocycle $\Phi$ is homologically trivial if and only if there exists an $S$-graded $\bigoplus_{i \in A} M_{i}$-module structure on $\bigoplus_{s \in S} M_{s}$ whose component structure maps span the $\mathcal{T}$-module datum. The action of 1-cochains, when restricted to 1-cocycles, induces an action of $H^{1}\left(A, F u n\left(S, \mathbb{C}^{\times}\right)\right)$on isomorphism classes of $S$-graded modules with fixed graded pieces. The action of 0 -cochains by rescaling modules induces an action of the 0 -cocycle group $F u n\left(S, \mathbb{C}^{\times}\right)^{A}$ by automorphisms of the $S$-graded module.

Proof. By essentially the same argument as the proof of Lemma 2.1.5, we have

$$
F(i, j, k) \Phi(i, j+k, s) \Phi(j, k, s)=\Phi(i+j, k, s) \Phi(i, j, k+s)
$$

However, we assume $F(i, j, k)=1$ for all $i, j, k \in A$, so we obtain the identity we want.

By examining the definition of $\Phi$, we see that rescaling the intertwining operators by some 1-cochain $\left\{\lambda_{i, s}\right\}_{i \in A, s \in S}$ multiplies $\Phi$ by the coboundary $d \lambda$, where $(d \lambda)(i, j, s)=\frac{\lambda_{i, j+s} \lambda_{j, s}}{\lambda_{i+j, s}}$.

$\Phi$ is homologically trivial if and only if there is a 1-cochain $\lambda$ such that $d \lambda \cdot \Phi \stackrel{=}{=}$, which is equivalent to the maps $\left\{\lambda_{i, s} m_{z}^{i, s}\right\}$ inducing a module structure.

If $\Phi$ is homologically trivial, then translation by the coboundary of a 1-cocycle $\left\{\lambda_{i, s}\right\}$ preserves the triviality of the $\Phi$, so we have an action of 1-cocycles on isomorphism classes of modules. 1coboundaries lie in the kernel of this action, since they amount to rescaling pieces of the module, i.e., there is a block diagonal matrix that induces a module isomorphism. The automorphisms induced by 0 -cocycles are just rescalings on $A$-orbits in $S$.

Definition 2.4.3. Let $V$ be a $\mathcal{T}$-vertex algebra, let $A$ be an abelian group, let $M_{A}=\bigoplus_{i \in A}$ be a $\mathcal{T}$-vertex algebra given by a $\mathcal{T}$-commutativity datum on $V$ modules $\left\{M_{i}\right\}_{i \in A}$, let $+: S_{1} \times S_{2} \rightarrow S_{3}$ be an $A$-equivariant map of $A$-sets (written as addition), and let $M_{S_{1}}, M_{S_{2}}, M_{S_{3}}$ be $M_{A}$-modules given by $\mathcal{T}$-module data. We define a $\mathcal{T}$-intertwining operator datum to be a set $\left\{\mathcal{I}_{r, s}^{r+s}\right\}_{r \in S_{1}, s \in S_{2}}$ of one-dimensional vector spaces, whose elements are $\mathcal{T}$-intertwining operators $M_{r} \otimes M_{s} \rightarrow M_{r+s}((z))$, such that:

(1) For each $i \in A, r \in S_{1}, s \in S_{2}$, there exists a one dimensional vector space $\mathcal{I}_{i, r, s}^{i+r+s}$ whose elements are maps $M_{i} \otimes M_{r} \otimes M_{s} \rightarrow M_{i+r+s}[[w, z]]\left[w^{-1}, z^{-1},(w-z)^{-1}\right]$, such that composition of intertwining operators, combined with the canonical injections from Lemma 1.1.4. induces isomorphisms from $\mathcal{I}_{i, r+s}^{i+r+s} \otimes \mathcal{I}_{r, s}^{r+s}$ to $\mathcal{I}_{i, r, s}^{i+r+s}$ and from $\mathcal{I}_{i+r, s}^{i+r+s} \otimes \mathcal{I}_{i, r}^{i+r}$ to $\mathcal{I}_{i, r, s}^{i+r+s}$.

(2) For any $i, j \in A, r \in S_{1}, s \in S_{2}$, there exists a one-dimensional space $\mathcal{I}_{i, j, r, s}^{i+j+r+s}$ whose elements are maps:

$$
M_{i} \otimes M_{j} \otimes M_{r} \otimes M_{s} \rightarrow M_{i+j+r+s}[[z, w, t]]\left[z^{-1}, w^{-1} t^{-1},(z-w)^{-1},(z-t)^{-1},(w-t)^{-1}\right]
$$

such that composition of intertwining operators, combined with one of the canonical injections from Lemma 1.1.6, induces an isomorphism from $\mathcal{I}_{i, j+r+s}^{i+j+r+s} \otimes \mathcal{I}_{j, r+s}^{j+r+s} \otimes \mathcal{I}_{r, s}^{r+s}$ to $\mathcal{I}_{i, j, r, s}^{i+j+r+s}$. 
Given a $\mathcal{T}$-intertwining operator datum and any choice of nonzero intertwining operators $\left\{m_{z}^{r, s} \in\right.$ $\left.\mathcal{I}_{r, s}^{r+s}\right\}_{r \in S_{1}, s \in S_{2}}$, we define the function $\Psi: A \times S_{1} \times S_{2} \rightarrow \mathbb{C}^{\times}$by

$$
m_{z}^{i, r+s} \circ\left(1 \otimes m_{w}^{r, s}\right)=\Psi(i, r, s) m_{w}^{i+r, s} \circ\left(m_{z-w}^{i, r} \otimes 1\right)
$$

Lemma 2.4.4. Given a $\mathcal{T}$-intertwining operator datum, and any choice of nonzero intertwining operators $\left\{m_{z}^{r, s} \in \mathcal{I}_{r, s}^{r+s}\right\}_{r \in S_{1}, s \in S_{2}}$, the function $\Psi$ satisfies the identity:

$$
\Psi(i, j+r, s) \Psi(j, r, s)=\Psi(i+j, r, s)
$$

In particular, $\Psi$ is a 1-cocycle for $A$ with coefficients in the A-module Fun $\left(S_{1} \times S_{2}, \mathbb{C}^{\times}\right)$, where the $A$-action on $S_{1} \times S_{2}$ is by $a+\left(s_{1}, s_{2}\right)=\left(a+s_{1}, s_{2}\right)$, i.e., we choose a trivial $A$-action on $S_{2}$. Rescaling the intertwining operators $m_{z}^{r, s}$ induces an action of the group of 0-cochains, by 1-coboundary translations. 0-cocycles are precisely the elements that leave $\Psi$ unchanged, and in particular, the degree zero cohomology group Fun $\left(S_{1} \times S_{2}, \mathbb{C}^{\times}\right)^{A}$ acts on the space of intertwining operators assembled from $\left\{m_{z}^{r, s}\right\}$.

Proof. By essentially the same argument as the proof of Lemma 2.1.5, we find that

$$
\Phi(i, j, r) \Psi(i, j+r, s) \Psi(j, r, s)=\Psi(i+j, r, s) \Phi(i, j, r+s)
$$

Since we assume $\Phi$ is identically 1 , we obtain the identity we want. By examining the definition of $\Psi$, we see that rescaling the intertwining operators by some 0 -cochain $\left\{\lambda_{r, s}\right\}_{r \in S_{1}, s \in S_{2}}$ multiplies $\Psi$ by the coboundary $d \lambda$, where $(d \lambda)(i, r, s)=\frac{\lambda_{i+r, s}}{\lambda_{r, s}}$.

Proposition 2.4.5. Let $\left\{\mathcal{I}_{i, j}^{i+j}\right\}_{i, j \in A}$ be a $\mathcal{T}$-commutativity datum, and let $B \subset A$ be a subgroup such that the quadratic form $i \mapsto \Omega(i, i)$ is constant on cosets of $B$. Then for any choice of $\mathcal{T}$-vertex algebra $M_{B}$ attached to the commutativity datum, the coset sums $M_{B+k}=\bigoplus_{i \in B+k} M_{i}$ admit the structure of $M_{B}$-modules in $\mathcal{T}$, such that for each $i, j \in A$, the restriction of the action to $M_{i} \otimes M_{j}$ lands $\mathcal{I}_{i, j}^{i+j}$. Furthermore, there exists a one-dimensional commutativity datum $\left\{\mathcal{I}_{B+i, B+j}^{B+i+j}\right\}$ on $\left\{M_{B+k}\right\}$ whose restriction to $\left\{M_{i} \otimes M_{j}\right\}$ spans the commutativity datum $\left\{\mathcal{I}_{i, j}^{i+j}\right\}_{i, j \in A}$.

Proof. By Lemma 1.2.7, $i \mapsto \Omega(i, i)$ is the pullback of a quadratic form on $A / B$, and there is a representing normalized cocycle $(F, \Omega)$ pulled back from a normalized abelian 3-cocycle $(\bar{F}, \bar{\Omega})$ on $A / B$. By the transitivity claim in Theorem 2.2.13, there exists a choice of intertwining operators $m_{z}^{i, j}$ representing $(F, \Omega)$. We claim that from this choice, we obtain a module structure on $M_{B+k}$ for each coset $B+k$, and intertwining operators between such modules that span a commutativity datum.

For the module structure, the obstruction 2-cocycle $\Phi$ vanishes, as it is the restriction of $F$ to $B \times B \times(B+k)$, hence the pullback of the normalized cocycle $\bar{F}$ on $(0,0, k)$.

To show that a map $M_{B+i} \otimes M_{B+j} \rightarrow M_{B+i+j}((z))$ is an intertwining operator, we note that the obstruction 1-cocycle $\Psi$ vanishes, as it is the restriction of $F$ to $B \times(B+i) \times(B+j)$, hence the pullback of the normalized cocycle $\bar{F}$ on $(0, i, j)$.

To see that these intertwining operators span an associativity datum, we note that each space $\mathcal{I}_{B+i, B+j, B+k}^{B+i+j+k}$ is naturally spanned by composites of the intertwining operators we constructed, and the composites differ by the scalar $\bar{F}(B+i, B+j, B+k)$. To see that this associativity datum is a commutativity datum for $\left\{M_{B+i}\right\}_{B+i \in A / B}$, we note that skew-symmetry follows from the normalization of $\bar{\Omega}$, i.e., $\bar{\Omega}(0, B+i)=1$.

Corollary 2.4.6. Any $\mathcal{T}$-commutativity datum for $\left\{M_{i}\right\}_{i \in A}$, induces for each coset $2 A+k$ in $A$, and each associated $\mathcal{T}$-vertex algebra structure $M_{2 A}$, an $M_{2 A}$-module structure on the sum $M_{2 A+k}=$ $\bigoplus_{i \in 2 A+k} M_{i}$, and a $\mathcal{T}$-commutativity datum for the resulting spaces of intertwining operators.

Proof. By Lemma 1.2.8, any \pm 1 -valued quadratic form on $A$ is identically 1 on $2 A$, and constant on cosets of $2 A$. The $\mathcal{T}$-commutativity datum then satisfies the conditions of Proposition 2.4.5. 


\section{QUASI-SIMPLE CURRENT EXTENSIONS}

Now that we have a good theory for constructing vertex algebras and modules from pieces, we'd like to prove existence theorems with fewer hypotheses about the concrete structure of intertwining operators, since they are in general difficult to compute. Instead, we shall make increasingly strong assumptions on the modules and their categories, and we will find that we can make much stronger conclusions about existence. Simple currents are ideal for this, since the intertwining operator structure is somehow minimal, and the commutativity datum exists essentially automatically.

Since we are considering vertex algebras that are not necessarily rational vertex operator algebras, we use slightly more general language, appending the prefix "quasi" make the distinction. In particular, our quasi-simple currents are not quite as well-behaved, as they lack some of the finiteness assumptions that appear in the literature.

In addition to our type $\mathcal{T}$, we will now introduce a new datum, restricting our view to a full subcategory $\mathcal{C}$ of simple $V$-modules in $\mathcal{T}$. This will help us manage fusion, since the full structure of the $V$-module category may be beyond our reach. Choosing $\mathcal{C}$ in concrete situations can be rather delicate: if we make $\mathcal{C}$ too big, our subcategory of quasi-simple currents may collapse to triviality, and if we make $\mathcal{C}$ too small, we may not get interesting phenomena in the first place.

\subsection{Restricted quasi-simple currents.}

Definition 3.1.1. Suppose $V$ is a simple $\mathcal{T}$-vertex algebra, and let $\mathcal{C}$ be a full subcategory of the category of $V$-modules in $\mathcal{T}$, such that all objects in $\mathcal{C}$ are simple $V$-modules. We define a quasisimple $\mathcal{C}$-current to be an object $M$ in $\mathcal{C}$ such that for any object $N$ in $\mathcal{C}$, there exists an object $X$ in $\mathcal{C}$ and a nonzero $\mathcal{T}$-intertwining operator $m_{z}^{M, N}: M \otimes N \rightarrow X((z))$, such that substitution of coefficients induces isomorphisms $\operatorname{Hom}_{V}(X, Y) \cong I_{\mathcal{T}}\left(\begin{array}{c}Y \\ M, N\end{array}\right)$ for all objects $Y$ in $\mathcal{C}$. In this case, we write $M \otimes N$ (or $M \otimes_{\mathcal{C}} N$ if there is the possibility of confusion) to denote the object $X$, and call the pair $\left(M \otimes N, m_{z}^{M, n}\right)$ the relative fusion product of $M$ and $N$ in $\mathcal{C}$.

Lemma 3.1.2. The isomorphism classes of objects in $\mathcal{C}$ form a set.

Proof. Any irreducible $V$-module is an irreducible module for the Frenkel-Zhu enveloping associative algebra of $V$ [Frenkel-Zhu 1992. Isomorphism classes of irreducible modules of an associative algebra can be identified with a subquotient of the power set of the algebra, since any such module is generated by some vector $m$, and hence isomorphic to the quotient by the maximal left ideal $\operatorname{Ann}(m)$.

Remark 3.1.3. Let $M$ be a quasi-simple current, and let $X=M \otimes N$ as in Definition 3.1.1. Skewsymmetry implies all intertwining operators $N \otimes M \rightarrow X((z))$ are scalar multiples of $m_{z}^{M, N, *}$. In particular, we have canonical isomorphisms $\operatorname{Hom}_{V}(X, Y) \cong I_{\mathcal{T}}\left(\begin{array}{c}Y \\ N, M\end{array}\right)$.

Definition 3.1.4. A pair $(M, N)$ of quasi-simple $\mathcal{C}$-currents is called composable if the following conditions hold:

(1) $M \otimes N$ is a quasi-simple $\mathcal{C}$-current.

(2) For any object $P$ in $\mathcal{C}, M \otimes(N \otimes P) \cong(M \otimes N) \otimes P$.

(3) For any object $P$ in $\mathcal{C}$, the composites $m_{z}^{M, N \otimes P} \circ\left(1 \otimes m_{w}^{N, P}\right)$ and $m_{w}^{M \otimes N, P} \circ\left(m_{z-w}^{M, N} \otimes 1\right)$ factor through the inclusions from $(M \otimes(N \otimes P))[[z, w]]\left[z^{-1}, w^{-1},(z-w)^{-1}\right]$ to $(M \otimes$ $(N \otimes P))((z))((w))$ and $(M \otimes(N \otimes P))((w))((z-w))$, and the two maps $M \otimes N \otimes P \rightarrow$ $M \otimes(N \otimes P)[[z, w]]\left[z^{-1}, w^{-1},(z-w)^{-1}\right]$ are scalar multiples of each other.

Lemma 3.1.5. If $\left(M_{1}, M_{2}\right)$ is a composable pair, then for any irreducible module $M_{3}$, the following four modules are isomorphic: $M_{1} \otimes\left(M_{2} \otimes M_{3}\right),\left(M_{1} \otimes M_{2}\right) \otimes M_{3},\left(M_{2} \otimes M_{1}\right) \otimes M_{3}, M_{2} \otimes\left(M_{1} \otimes M_{3}\right)$.

Proof. By skew-symmetry, $M_{1} \otimes M_{2} \cong M_{2} \otimes M_{1}$, so the notion of composable pair is independent of order. In particular, the second and third modules are isomorphic. By the definition of composable pair, the first two modules are isomorphic, and the last two modules are hence isomorphic. 
Lemma 3.1.6. If $\left(M_{1}, M_{2}\right)$ is a composable pair, then for any irreducible module $M_{3}$ and elements $m_{i} \in M_{i}, m_{z}^{M_{1}, M_{2} \otimes M_{3}} \circ\left(1 \otimes m_{w}^{M_{2}, M_{3}}\right)\left(m_{1} \otimes m_{2} \otimes m_{3}\right)$ is a constant multiple of $m_{w}^{M_{2}, M_{1} \otimes M_{3}} \circ(1 \otimes$ $\left.m_{z}^{M_{1}, M_{3}}\right)\left(m_{2} \otimes m_{1} \otimes m_{3}\right)$ in $M_{1} \otimes\left(M_{2} \otimes M_{3}\right)[[z, w]]\left[z^{-1}, w^{-1},(z-w)^{-1}\right]$. In particular, there is some positive integer $N$ such that multiplication by $z^{N} w^{N}(z-w)^{N}$ gives proportional elements in $M_{1} \otimes\left(M_{2} \otimes M_{3}\right)[[z, w]]$.

Proof. This is essentially the standard argument that skew-symmetry and associativity imply locality.

Definition 3.1.7. A quasi-simple $\mathcal{C}$-current is universally composable if it forms composable pairs with all quasi-simple $\mathcal{C}$-currents. We write $\operatorname{Curr}_{\mathcal{C}}(V)$ for the full subcategory of universally composable quasi-simple $\mathcal{C}$-currents.

Lemma 3.1.8. Suppose $M_{1}$ and $M_{2}$ are universally composable quasi-simple $\mathcal{C}$-currents, and $M_{3}$ is a quasi-simple $\mathcal{C}$-current. For any object $P$ in $\mathcal{C}$, and any permutation $\sigma$ of $\{1,2,3\}$, let $X_{\sigma}=$ $M_{\sigma(1)} \otimes\left(M_{\sigma(2)} \otimes\left(M_{\sigma(3)} \otimes P\right)\right)$. Then all $X_{\sigma}$ are isomorphic, and all composites

$$
m_{z_{\sigma(3)}}^{M_{\sigma(1)}, M_{\sigma(2)} \otimes\left(M_{\sigma(3)} \otimes P\right)} \circ\left(1 \otimes m_{z_{\sigma(2)}}^{M_{\sigma(2)}, M_{\sigma(3)} \otimes P}\right) \circ\left(1 \otimes 1 \otimes m_{z_{\sigma(1)}}^{M_{\sigma(3)}, P}\right)
$$

factor through the embeddings

$$
X_{1}\left[\left[z_{1}, z_{2}, z_{3}\right]\right]\left[z_{1}^{-1}, z_{2}^{-1}, z_{3}^{-1},\left(z_{1}-z_{2}\right)^{-1},\left(z_{1}-z_{3}\right)^{-1},\left(z_{2}-z_{3}\right)^{-1}\right] \rightarrow X_{\sigma}\left(\left(z_{\sigma(3)}\right)\right)\left(\left(z_{\sigma(2)}\right)\right)\left(\left(z_{\sigma(1)}\right)\right) .
$$

Furthermore, the induced maps $M_{1} \otimes M_{2} \otimes M_{3} \otimes P \rightarrow X_{1}\left[\left[z_{1}, z_{2}, z_{3}\right]\right]\left[z_{1}^{-1}, z_{2}^{-1}, z_{3}^{-1},\left(z_{1}-z_{2}\right)^{-1},\left(z_{1}-\right.\right.$ $\left.z_{3}\right)^{-1},\left(z_{2}-z_{3}\right)^{-1}$ ] are scalar multiples of each other.

Proof. The isomorphisms between the $X_{\sigma}$ follow from repeatedly applying Lemma 3.1.5.

For each permutation $\sigma$, we consider an element $p_{\sigma}=m_{\sigma(1)} \otimes m_{\sigma(2)} \otimes m_{\sigma(3)} \otimes p \in M_{\sigma(1)} \otimes M_{\sigma(2)} \otimes$ $M_{\sigma(3)} \otimes P$, and let $x_{\sigma}=m_{z_{\sigma(3)}}^{M_{\sigma(1)}, M_{\sigma(2)} \otimes\left(M_{\sigma(3)} \otimes P\right)} \circ\left(1 \otimes m_{z_{\sigma(2)}}^{M_{\sigma(2)}, M_{\sigma(3)} \otimes P}\right) \circ\left(1 \otimes 1 \otimes m_{z_{\sigma(1)}}^{M_{\sigma(3)}, P}\right)\left(p_{\sigma}\right)$

Locality, as in Lemma 3.1.6, implies that for a sufficiently large positive integer $N$, the series $\left(z_{1}-z_{2}\right)^{N}\left(z_{1}-z_{3}\right)^{N}\left(z_{2}-z_{3}\right)^{N} x_{\sigma}$ are independent of $\sigma$ up to scalar multiplication. In particular, poles in the variables $z_{1}, z_{2}, z_{3}$ are uniformly bounded in order. This implies the existence of the factorization.

Lemma 3.1.9. The fusion product of two universally composable quasi-simple $\mathcal{C}$-currents is universally composable.

Proof. Let $M_{1}, M_{2}$, and $M_{3}$ be quasi-simple $\mathcal{C}$-currents, and suppose $M_{1}$ and $M_{2}$ are universally composable. We wish to show that $M_{1} \otimes M_{2}$ and $M_{3}$ form a composable pair.

For the first condition, we need to show that $\left(M_{1} \otimes M_{2}\right) \otimes M_{3}$ is a quasi-simple $\mathcal{C}$-current, and by Lemma 3.1.5, it suffices to show that $M_{1} \otimes\left(M_{2} \otimes M_{3}\right)$ is a quasi-simple $\mathcal{C}$-current. Since $M_{2}$ is universally composable, $M_{2} \otimes M_{3}$ is a quasi-simple $\mathcal{C}$-current, and since $M_{1}$ is universally composable, $M_{1} \otimes\left(M_{2} \otimes M_{3}\right)$ is a quasi-simple $\mathcal{C}$-current.

For the second condition, we alternately use the universally composable properties of $M_{1}$ and $M_{2}$ to obtain the following isomorphisms:

$$
\begin{aligned}
\left(M_{1} \otimes M_{2}\right) \otimes\left(M_{3} \otimes P\right) & \cong M_{1} \otimes\left(M_{2} \otimes\left(M_{3} \otimes P\right)\right) \\
& \cong M_{1} \otimes\left(\left(M_{2} \otimes M_{3}\right) \otimes P\right) \\
& \cong\left(M_{1} \otimes\left(M_{2} \otimes M_{3}\right)\right) \otimes P \\
& \cong\left(\left(M_{1} \otimes M_{2}\right) \otimes M_{3}\right) \otimes P
\end{aligned}
$$

For the last condition, we shall write $M^{\prime}=\left(\left(M_{1} \otimes M_{2}\right) \otimes M_{3}\right) \otimes P$ for convenience. We need to show that the composites $m^{1}=m_{w}^{M_{1} \otimes M_{2}, M_{3} \otimes P} \circ\left(1 \otimes m_{t}^{M_{3}, P}\right)$ and $m^{2}=m_{t}^{\left(M_{1} \otimes M_{2}\right) \otimes M_{3}, P} \circ$ $\left(m_{w-t}^{M_{1} \otimes M_{2}, M_{3}} \otimes 1\right)$ factor through the inclusions from $M^{\prime}[[w, t]]\left[w^{-1}, t^{-1},(w-t)^{-1}\right]$ to $M^{\prime}((w))((t))$ 
and $M^{\prime}((t))((w-t))$, and that the two maps $\left(M_{1} \otimes M_{2}\right) \otimes M_{3} \otimes P \rightarrow M^{\prime}[[w, t]]\left[w^{-1}, t^{-1},(w-t)^{-1}\right]$ are scalar multiples of each other. By the irreducibility of $M_{1} \otimes M_{2}$, it suffices to show this for all coefficients of $(z-w)^{n}$ in $m_{z-w}^{M_{1}, M_{2}} \otimes 1 \otimes 1$ applied to $M_{1} \otimes M_{2} \otimes M_{3} \otimes P$.

We use the notation of Lemma 1.1.6, but with $M^{\prime}$ in place of $V$. Pre-composing both $m^{1}$ and $m^{2}$ with $m_{z-w}^{M_{1}, M_{2}}$, we obtain a map $\hat{m}^{1}$ to $M_{(i j)(k \ell)}^{\prime}$ and a map $\hat{m}^{2}$ to $M_{((i j) k) \ell}^{\prime}$. It therefore suffices to show that both factor through maps to $M_{[(i j) k] \ell}^{\prime}$ that are scalar multiples of each other.

By Lemma 3.1.8, the composite $\hat{m}^{3}=m_{z}^{M_{1}, M_{2} \otimes\left(M_{3} \otimes P\right)} \circ\left(1 \otimes m_{w}^{M_{2}, M_{3} \otimes P}\right) \circ\left(1 \otimes 1 \otimes m_{t}^{M_{3}, P}\right)$ factors through $M_{[i j k] \ell}^{\prime}$. By repeatedly applying Lemma 3.1.6, we see that $\hat{m}^{1}$ and $\hat{m}^{2}$ also factor through maps to $M_{[i j k] \ell}^{\prime}$, and are proportional to the corresponding map that factors $\hat{m}^{3}$. Using the embedding $M_{[i j k] \ell}^{\prime} \rightarrow M_{[(i j) k] \ell}^{\prime}$, we see that $\hat{m}^{1}$ and $\hat{m}_{2}$ factor through maps to $M_{[(i j) k] \ell}^{\prime}$ that are proportional.

Definition 3.1.10. A $\mathcal{T}$-vertex algebra $V$ is a $\mathcal{C}$-unit if it is an object in $\mathcal{C}$, and if it satisfies $V \otimes M \cong M$ for any object $M$ in $\mathcal{C}$. In particular, it is a quasi-simple $\mathcal{C}$-current.

Proposition 3.1.11. Suppose $V$ is a $\mathcal{C}$-unit. Then $C \operatorname{Curr}_{\mathcal{C}}(V)$ is a $\mathbb{C}$-linear symmetric monoidal category under fusion product $(M, N) \mapsto M \otimes N$.

Proof. The associator $a_{M N P}:(M \otimes N) \otimes P \rightarrow M \otimes(N \otimes P)$ is the proportionality constant between the three-input intertwining operators given by composites.

By combining Lemma 3.1.8 and Lemma 3.1.6, the compositions of intertwining operators given in the pentagon diagram are proportional, so the associator satisfies the pentagon axiom.

The unit structures $\ell_{M}: V \otimes M \rightarrow M$ and $r_{M}: M \otimes V \rightarrow M$ are canonically given by the action of $V$, and the intertwining operator induced by skew-symmetry.

The identity $r_{M} \otimes 1=\left(1 \otimes \ell_{N}\right) \circ a_{M V N}$ is straightforward.

The commutor is the proportionality constant between $m_{z}^{M, N}$ and $m_{z}^{N, M, *}$. This is automatically symmetric, since intertwining operators are integer weight.

By combining Lemma 3.1.8 and Lemma 3.1.6, the compositions of intertwining operators given in the hexagon diagrams are proportional, so the commutor and associator satisfy the hexagon axioms.

From now on, we assume $V$ is a $\mathcal{C}$-unit.

Definition 3.1.12. An inverse of a universally composable quasi-simple $\mathcal{C}$-current $M$ is a universally composable quasi-simple $\mathcal{C}$-current $N$, such that $M \otimes N \cong V$ for $V$ a $\mathcal{C}$-unit. If $M$ admits an inverse, we say that $M$ is invertible. We define the Picard groupoid $\underline{\operatorname{Pic}}_{\mathcal{C}}(V)$ to be the category of invertible universally composable quasi-simple $\mathcal{C}$-currents, with morphisms given by isomorphisms in $\mathcal{C}$.

Lemma 3.1.13. $\underline{\operatorname{Pic}}_{\mathcal{C}}(V)$ is a symmetric monoidal subcategory of $C \operatorname{Curr}_{\mathcal{C}}(V)$.

Proof. It suffices to show that the fusion product of two invertible objects is invertible. If $M$ and $N$ are invertible universally composable $\mathcal{C}$-currents, then the associativity property of composable pairs implies $M \otimes N$ and $M^{-1} \otimes N^{-1}$ are inverses.

Remark 3.1.14. Note that this Picard groupoid is not necessarily a strictly commutative Picard category in the sense of [SGA4] Exp. XVIII Definition 1.4.2, since the commutor isomorphism on $M \otimes M$ is not necessarily identity.

Lemma 3.1.15. The isomorphism classes in $\underline{\operatorname{Pic}}_{\mathcal{C}}(V)$ form a set, and the monoidal structure induces an abelian group structure on this set.

Proof. The isomorphism classes in $\mathrm{Pic}_{\mathcal{C}}(V)$ are a subclass of the set of isomorphism classes in $\mathcal{C}$, so they form a set. $\pi_{0}$ of an essentially small symmetric monoidal category is an abelian monoid, and invertibility of objects implies invertibility of elements of the monoid. 
Definition 3.1.16. We call the group of isomorphism classes in $\underline{\operatorname{Pic}}_{\mathcal{C}}(V)$ the Picard group of $V$ in $\mathcal{C}$, and write $\operatorname{Pic}_{\mathcal{C}}(V)$. Given a homomorphism $A \rightarrow \operatorname{Pic}_{\mathcal{C}}(V)$ of abelian groups, we write $M_{i}$ for a module in $\mathcal{T}$ in the isomorphism class defined by $\pi(i)$. We say that a homomorphism $A \rightarrow \operatorname{Pic}_{\mathcal{C}}(V)$ is $\mathcal{T}$-compatible if the set of modules $\left\{M_{i}\right\}_{i \in A}$ is $\mathcal{T}$-compatible (in the sense of Definition 2.2.10).

Proposition 3.1.17. Let $\pi: A \rightarrow \operatorname{Pic}_{\mathcal{C}}(V)$ be a homomorphism of abelian groups. Then the modules $\left\{M_{i}\right\}_{i \in A}$ admit a canonical $\mathcal{T}$-commutativity datum.

Proof. Our spaces of intertwining operators are spanned by composites. Lemma 3.1.8 gives us our associativity datum. Skew-symmetry is more or less automatic.

Definition 3.1.18. Let $\pi: A \rightarrow \operatorname{Pic}_{\mathcal{C}}(V)$ be a homomorphism of abelian groups. Any $\mathcal{T}$-vertex algebra structure on $\bigoplus_{i \in A} M_{i}$ whose multiplication restricts to nonzero $\mathcal{T}$-intertwining operators $M_{i} \otimes M_{j} \rightarrow M_{i+j}((z))$ (which is unique up to isomorphism if it exists) is called the quasi-simple $\mathcal{C}$-current extension of $V$ by $\pi$.

Definition 3.1.19. A quasi-simple $\mathcal{C}$-current $M$ is even if some (equivalently, any) nonzero intertwining operator $M \otimes M \rightarrow(M \otimes M)((z))$ is even. A homomorphism $A \rightarrow \operatorname{Pic}_{\mathcal{C}}(V)$ is even if the image is spanned by even objects.

Remark 3.1.20. At our current level of generality, it seems that the even objects in the Picard groupoid of $V$ do not necessarily form a monoidal subcategory. At least, if $\operatorname{Pic}_{\mathcal{C}}(V) \cong \mathbb{Z} / 2 \mathbb{Z} \times \mathbb{Z} / 2 \mathbb{Z}$, I was unable to rule out the possibility that the quadratic form $\Omega$ is -1 on $(1,1)$ and 1 on all other elements.

Proposition 3.1.21. For any even homomorphism $\pi: A \rightarrow \operatorname{Pic}_{\mathcal{C}}(V)$, the canonical commutativity datum given in Proposition 3.1 .17 is even. In particular, if $\pi$ is $\mathcal{T}$-compatible, then there exists a quasi-simple $\mathcal{C}$-current extension $W_{A}=\bigoplus_{i \in A} M_{i}$ of $V$ by $\pi$, and its isomorphism type is uniquely determined.

Proof. The condition on $\pi$ forces this commutativity datum to be even. By Proposition 2.3.6, $W_{A}$ admits a $\mathcal{T}$-vertex algebra structure, whose isomorphism type is uniquely determined.

Lemma 3.1.22. Let $M$ be an object in $\mathcal{C}$. Then the subcategory $\operatorname{Stab}_{\mathcal{C}}(M)$ of $\underline{\operatorname{Pic}}_{\mathcal{C}}(V)$ whose objects $M_{i}$ satisfy $M_{i} \otimes M \cong M$ forms a Picard subgroupoid. For any module $M_{i}$ in $\underline{\text { Pic }}_{\mathcal{C}}(V)$, the isomorphism type of $M_{i} \otimes M$ depends only on the coset of the corresponding subgroup $\operatorname{Stab}_{\mathcal{C}}(M)$ in $\operatorname{Pic}_{\mathcal{C}}(V)$.

Proof. If $M_{i}$ and $M_{j}$ stabilize $M$, then $M_{i} \otimes\left(M_{j} \otimes M\right) \cong M_{i} \otimes M \cong M$, so we have a full symmetric monoidal subcategory. If $M_{i}$ stabilizes $M$, then $M \cong\left(M_{i}^{-1} \otimes M_{i}\right) \otimes M \cong M_{i}^{-1} \otimes\left(M_{i} \otimes M\right) \cong$ $M_{i}^{-1} \otimes M$, so this category is closed under inverses. If $M_{j}$ stabilizes $M$ but $M_{i}$ does not necessarily stabilize $M$, then $M_{i} \otimes M \cong M_{i} \otimes\left(M_{j} \otimes M\right) \cong\left(M_{i} \otimes M_{j}\right) \bigotimes M \cong M_{i+j} \otimes M$, so we have independence within cosets.

Lemma 3.1.23. Let $W_{A}$ denote the quasi-simple $\mathcal{C}$-current extension of $V$ by an even homomorphism $\pi: A \rightarrow \operatorname{Pic}_{\mathcal{C}}(V)$. Then for any object $M$ in $\mathcal{C}$, the direct sum $\bigoplus_{i \in A /\left(A \cap \operatorname{Stab}_{\mathcal{C}}(M)\right)}\left(M_{i} \otimes M\right)$ is an irreducible $W_{A}$-module in $\mathcal{T}$. In particular, $W_{A}$ is a simple $\mathcal{T}$-vertex algebra.

Proof. By the coset property of the stabilizer, the direct sum module is multiplicity-free as a $V$ module. Since each $M_{i} \otimes M$ is irreducible, any $W_{A}$-submodule $N$, when viewed as a $V$-module, is contained in the direct sum of the irreducible modules $M_{i} \otimes M$, so it is isomorphic to a direct sum of a subset of such modules that is closed under fusion with the constituent modules in $W_{A}$. Such a subset is either empty or the full coset space, so $N$ is either the whole direct sum module or zero. 
Recall from Corollary 2.4.6 that for any $\pi: A \rightarrow \operatorname{Pic}_{\mathcal{C}}(V)$, the subgroup $2 A$ plays a special role, in that we have existence of a vertex algebra $W_{2 A}$, and modules $M_{2 A+i}$ for each coset of $2 A$ in $A$, such that there is a commutativity datum on these modules induced from the commutativity datum on $V$-modules. If we choose our category $\mathcal{C}^{\prime}$ so that its objects are the $W_{2 A}$ modules $M_{2 A+i}$, we find that $\mathcal{C}^{\prime}=\operatorname{Curr}\left(\mathcal{C}^{\prime}\right)$ is symmetric monoidal, and the modules are quasi-simple $\mathcal{C}^{\prime}$-currents whose tensor square is $W_{2 A}$. Unfortunately, we cannot conclude that they are even, so we can only conclude that totally even subgroups of $A / 2 A$ induce $\mathcal{T}$-vertex algebras as quasi-simple $\mathcal{C}$-current extensions.

3.2. Contragradients and regularity. We continue to use the notation from the previous section, and we maintain the assumption that $V$ is a $\mathcal{C}$-unit. From now on, we shall assume that $\mathcal{T}$ incorporates Möbius structure (in particular, $V$ is Möbius, quasi-conformal, conformal, or a vertex operator algebra), and furthermore, that there is a contragradient involution on $\mathcal{C}$ (see Definition 3.2.1 below), such that $V \cong V^{\vee}$. Equivalently, we assume there is a nondegenerate invariant bilinear form on $V$.

Definition 3.2.1. Let $V$ be a Möbius (resp. quasiconformal, conformal) vertex algebra or vertex operator algebra. Given an integral-weight $V$-module $M$ in the Möbius (resp. quasiconformal, conformal, VOA) sense, a weak contragradient is a $V$-module $M^{\vee}$ equipped with a bilinear map $\langle-,-\rangle: M^{\vee} \times M \rightarrow \mathbb{C}$ satisfying the following properties:

(1) The pairing is non-degenerate, i.e., the left and right kernels are zero.

(2) Virasoro operators are adjoint, i.e., $\langle L(i) u, m\rangle=\langle u, L(-i) m\rangle$ for all $i \in \mathbb{Z}$ for which $L(i)$ and $L(-i)$ are defined.

(3) The $V$-action satisfies the adjoint identity from Frenkel-Huang-Lepowsky 1993 equation 5.2.4:

$$
\left\langle u, a c t_{z}(v \otimes m)\right\rangle=\left\langle a c t_{z^{-1}}\left(e^{z L(1)}\left(-z^{-2}\right)^{L(0)} v \otimes u\right), m\right\rangle
$$

for all $v \in V, m \in M, u \in M^{\vee}$.

Given a full subcategory $\mathcal{C}$ of $V$-modules in the Möbius (resp. quasiconformal, conformal, VOA) sense, a contragradient involution is a contravariant functor on $\mathcal{C}$ that takes each object to a weak contragradient $M \mapsto M^{\vee}$ on $\mathcal{C}$, and whose composite with itself is isomorphic to identity.

Remark 3.2.2. For vertex operator algebras, integral-weight modules have a natural contragradient functor given by taking the sum of dual spaces in each $L(0)$-eigenspace - this is involutive because the eigenspaces are finite dimensional. In more general settings, such a functor may require unnatural choices. However, Huang-Lepowsky-Zhang 2007 has a "strongly gradable" condition that seems to cover the known interesting examples, and implies the existence of a contragradient involution.

Definition 3.2.3. Given a $\mathcal{T}$-intertwining operator $I_{z}: M_{1} \otimes M_{2} \rightarrow M_{3}((z))$, its adjoint $I_{z}^{\prime}$ : $M_{1} \otimes M_{3}^{\vee} \rightarrow M_{2}^{\vee}((z))$ is defined by:

$$
\left\langle\phi, I_{z}(v \otimes m)\right\rangle=\left\langle I_{z^{-1}}^{\prime}\left(e^{z L(1)}\left(-z^{-2}\right)^{L(0)} v \otimes \phi\right), m\right\rangle
$$

for all $v \in M_{1}, m \in M_{2}$, and $\phi \in M_{3}^{\vee}$.

Remark 3.2.4. By Proposition 5.5.2 in Frenkel-Huang-Lepowsky 1993, passage to the adjoint intertwining operator yields an isomorphism $\mathcal{I}_{\mathcal{T}}\left(\begin{array}{c}M_{3} \\ M_{1}, M_{2}\end{array}\right) \cong \mathcal{I}_{\mathcal{T}}\left(\begin{array}{c}M_{2}^{\vee} \\ M_{1}, M_{3}^{\vee}\end{array}\right)$.

Lemma 3.2.5. For any quasi-simple $\mathcal{C}$-current $M$, we have $M \otimes M^{\vee} \cong V$. In particular, the contragradient involution restricts to an involution on $C \operatorname{Curr}_{\mathcal{C}}(V)$ and $\underline{\operatorname{Pic}}_{\mathcal{C}}(V)$.

Proof. Since $M$ is a simple current, $M \otimes M^{\vee}$ is an object of $\mathcal{C}$. By using the adjoint intertwining operator of $a c t_{z}^{*}$ and self-duality of $V$, we see that there exists a one-dimensional space of intertwining operators $M^{\vee} \otimes M \rightarrow V((z))$, spanned by the operator $I_{z}$ defined by:

$$
\left\langle v, I_{z}\left(m^{\prime} \otimes m\right)\right\rangle_{V}=\left\langle a c t_{-z^{-1}}\left(v \otimes e^{z L(1)}\left(-z^{2}\right)^{-L(0)} m^{\prime}\right), e^{z^{-1} L(1)} m\right\rangle_{M}
$$


for all $v \in V, m \in M, m^{\prime} \in M^{\vee}$. Thus, $M \otimes M^{\vee} \cong V$, and $M^{-1} \cong M^{\vee}$.

Remark 3.2.6. If $M \cong M^{\vee}$, then we get a contragradient pairing $\langle-,-\rangle_{M}$ on $M$, so the space $I_{\mathcal{T}}\left(\begin{array}{c}V \\ M, M\end{array}\right)$ of $\mathcal{T}$-intertwining operators $I: M \otimes M \rightarrow V((z))$ is one dimensional and spanned by the operator $I_{z}$ given above. This operator is put to good use in an alternative construction of the monster vertex algebra $V^{\natural}$, in section 3.4 of Huang 1994.

The following is a minor generalization of Proposition 2.6 in [Lam-Yamauchi 2006].

Proposition 3.2.7. Suppose $V$ is self-contragradient. Let $M$ be a self-dual object in $\mathcal{C}$. Then $M$ is even if and only if the contragradient pairing $\langle-,-\rangle_{M}$ is symmetric, and $M$ is odd if and only if the contragradient pairing is antisymmetric.

Proof. Let $m$ and $m^{\prime}$ be homogeneous of weight $k$. Then we have

$$
\begin{aligned}
\left\langle\mathbf{1}, I_{z}\left(m^{\prime} \otimes m\right)\right\rangle_{V} & =\left\langle a c t_{-z^{-1}}\left(\mathbf{1} \otimes e^{z L(1)}\left(-z^{2}\right)^{-L(0)} m^{\prime}\right), e^{z^{-1} L(1)} m\right\rangle_{M} \\
& =(-1)^{k} z^{-2 k}\left\langle e^{z L(1)} m^{\prime}, e^{z^{-1} L(1)} m^{\prime}\right\rangle_{M} \\
& =(-1)^{k} z^{-2 k} \sum_{j=0}^{\infty} \frac{1}{(j !)^{2}}\left\langle L(1)^{j} m^{\prime}, L(1)^{j} m\right\rangle_{M}
\end{aligned}
$$

Let $\Omega_{M}$ be the constant that is 1 if $M$ is even and -1 if $M$ is odd (this is commonly known as the Frobenius-Schur indicator of $M)$. The identity $\Omega_{M} I_{z}\left(m^{\prime} \otimes m\right)=I_{z}^{*}\left(m \otimes m^{\prime}\right)=e^{z L(-1)} I_{-z}\left(m \otimes m^{\prime}\right)$ implies

$$
\begin{aligned}
\left\langle\mathbf{1}, \Omega_{M} I_{z}\left(m^{\prime} \otimes m\right)\right\rangle_{V} & =\left\langle\mathbf{1}, e^{z L(-1)} I_{-z}\left(m \otimes m^{\prime}\right)\right\rangle_{V} \\
& =\left\langle e^{z L(1)} \mathbf{1}, I_{-z}\left(m \otimes m^{\prime}\right)\right\rangle_{V} \\
& =\left\langle\mathbf{1}, I_{-z}\left(m \otimes m^{\prime}\right)\right\rangle_{V}
\end{aligned}
$$

We conclude that

$$
\Omega_{M} \sum_{j=0}^{\infty} \frac{1}{(j !)^{2}}\left\langle L(1)^{j} m^{\prime}, L(1)^{j} m\right\rangle_{M}=\sum_{j=0}^{\infty} \frac{1}{(j !)^{2}}\left\langle L(1)^{j} m, L(1)^{j} m^{\prime}\right\rangle_{M}
$$

Note that the restriction of $\langle-,-\rangle_{M}$ to the primary subspace, where $L(1)$ acts as zero, is clearly symmetric when $M$ is even, and antisymmetric when $M$ is odd. This is a rather superfluous base of our induction, but it helps to illustrate the point. For each positive integer $k$, let $M_{[k]}$ denote the subspace of $M$ annihilated by $L(1)^{k}$, and let $P_{k}$ be the claim that the restriction of $\langle-,-\rangle_{M}$ to $M_{[k]}$ is symmetric when $M$ is even, and antisymmetric when $M$ is odd. If $P_{n}$ holds for some $n$, then for any $m, m^{\prime}$ in $M_{[n+1]}$,

$$
\begin{aligned}
\Omega_{M}\left\langle m^{\prime}, m\right\rangle_{M}+\Omega_{M} \sum_{j=1}^{n} \frac{1}{(j !)^{2}}\left\langle L(1)^{j} m^{\prime}, L(1)^{j} m\right\rangle_{M} & =\Omega_{M} \sum_{j=0}^{n} \frac{1}{(j !)^{2}}\left\langle L(1)^{j} m^{\prime}, L(1)^{j} m\right\rangle_{M} \\
& =\sum_{j=0}^{n} \frac{1}{(j !)^{2}}\left\langle L(1)^{j} m, L(1)^{j} m^{\prime}\right\rangle_{M} \\
& =\left\langle m, m^{\prime}\right\rangle_{M}+\sum_{j=1}^{n} \frac{1}{(j !)^{2}}\left\langle L(1)^{j} m, L(1)^{j} m^{\prime}\right\rangle_{M}
\end{aligned}
$$

By our inductive hypothesis, we can cancel the sums with positive $j$, so $\Omega_{M}\left\langle m^{\prime}, m\right\rangle_{M}=\left\langle m, m^{\prime}\right\rangle_{M}$, and $P_{n+1}$ holds. By the local nilpotence axiom on modules, the increasing filtration of $M$ by $\left\{M_{[k]}\right\}_{k \geq 0}$ is exhaustive, so $M$ even implies $\langle-,-\rangle_{M}$ is symmetric, and $M$ odd implies $\langle-,-\rangle_{M}$ is antisymmetric. $M$ is either even or odd, and inner product is nondegenerate, hence it cannot be both symmetric and antisymmetric. This yields the converse implications. 
Corollary 3.2.8. Suppose there exists a contragradient involution on a subcategory $\mathcal{C}$ of irreducible $V$-modules in $\mathcal{T}$, and suppose $V$ is self-contragradient. Let $\pi: A \rightarrow \operatorname{Pic}_{\mathcal{C}}(V)$ be a $\mathcal{T}$-compatible homomorphism from an $\mathbb{F}_{2}$-vector space. Then the canonical commutativity datum given in Proposition 3.1 .17 is even if and only if the contragradient form on each module is symmetric. In particular, there exists a quasi-simple $\mathcal{C}$-current extension of $V$ by $\pi$ if and only if all of the contragradient forms are symmetric.

Proof. The objects in this category are their own contragradients, since the contragradient on $\mathcal{C}$ permutes the component $V$-modules in each $M_{2 A+i}$. In particular, $V$ is self-contragradient, so by Proposition 3.2.7, an object in $\mathcal{C}^{\prime}$ is even if and only if its contragradient form is symmetric. Thus, our new commutativity datum is even, and Proposition 2.3.6 yields existence of the quasi-simple $\mathcal{C}$-current extension of $V$ by $\pi$, if and only if each contragradient form is symmetric..

At this point, we refine our scope again. If our Möbius vertex algebra $V$ satisfies sufficiently strong finiteness hypotheses, then the category of $V$-modules is a braided monoidal category. Among other benefits, this means we no longer have to make assumptions about the composability of intertwining operators. Because of the braided monoidal structure, we have a commutative fusion ring $K_{0}(V)$, whose product encodes the tensor structure of the semisimplification. Simple modules form a distinguished basis of $K_{0}(V)$, and simple currents (in the sense of [Dong-Li-Mason 1995]) are the modules represented by units in the fusion ring. For this purpose, we adopt the following:

Convention: We choose $V$ to be a simple $C_{2}$-cofinite Möbius vertex algebra with finite dimensional weight spaces and invariant bilinear form, and $\mathcal{C}$ to be any full subcategory of the category of $V$-modules, whose objects have integral weight, such that the isomorphism classes span a multiplicative subgroup of the fusion ring. As a special case, we may take $V$ to be a $C_{2}$-cofinite vertex operator algebra.

Lemma 3.2.9. If $V$ is a simple $C_{2}$-cofinite Möbius vertex algebra with finite dimensional weight spaces, then the category of $V$-modules is braided monoidal, with finitely many simple objects.

Proof. By Theorem 12.13 of [Huang-Lepowsky-Zhang 2007], to get a braided monoidal structure, it suffices to show that their Assumption 12.1 holds, but by their Theorem 11.5, it suffices to show that all $V$-modules are $C_{1}$-cofinite, and the quasi-finite dimensionality condition holds. Theorem 13 of Gaberdiel-Neitzke 2000] implies both conditions, but under the hypothesis that $V$ is a vertex operator algebra. However, the proof only uses the assumption that $V$ has a weighted vertex algebra structure with finite dimensional weight spaces and weights that are bounded below.

Lemma 3.2.10. Under our convention on $\mathcal{C}$, all objects are invertible universally composable quasisimple $\mathcal{C}$-currents. In particular, $\underline{\operatorname{Pic}}_{\mathcal{C}}(V)$ is the core of $\mathcal{C}$, i.e., the subcategory with the same objects, but only the invertible morphisms.

Proof. Because the corresponding elements in the fusion ring are invertible, fusion with any object necessarily takes irreducibles to irreducibles. Thus, any object of $\mathcal{C}$ is a quasi-simple $\mathcal{C}$-current. Universal composability holds by the compatibility of composites and iterates of intertwining operators (Assumption 12.1 in Huang-Lepowsky-Zhang 2007).

Definition 3.2.11. A unit in the fusion ring of $V$ is called even if it corresponds to an even quasi-simple $\mathcal{C}$-current.

Theorem 3.2.12. Let $V$ be a simple $C_{2}$-cofinite Möbius vertex algebra with finite dimensional weight spaces and invariant bilinear form. For any collection of $V$-modules parametrized by a group of even units in the fusion ring of $V$, the direct sum has the structure of a quasi-simple $\mathcal{C}$-current extension, and this structure is unique up to isomorphism. The extension is a $C_{2}$-cofinite Möbius vertex algebra with finite dimensional weight spaces. If $V$ is also a rational vertex operator algebra, then the extension is also rational, i.e., it is a simple regular vertex operator algebra. 
Proof. The first claim follows from Proposition 3.1.21. $C_{2}$-cofiniteness follows from Lemma 2.6 in Yamauchi 2002 - the arguments in the cited papers use only the weighted structure on $V$. Rationality follows from either Theorem 4.5 of [Lam 2001] or Theorem 2.14 of [Yamauchi 2002].

As we mentioned in the introduction, we think evenness always holds under our current convention, although the weight of evidence is somewhat stronger in the case of regular VOAs than for $C_{2}$-cofinite Möbius vertex algebras.

Conjecture 3.2.13 (Evenness conjecture). For any simple self-dual module $M$ over a simple $C_{2}$-cofinite Möbius vertex algebra with invariant bilinear form, the contragradient form on $M$ is symmetric.

Theorem 3.2.14. Assuming the evenness conjecture, any collection of integral-weight modules parametrized by a group of units in the fusion ring of $V$ admits the structure of a quasi-simple $\mathcal{C}$-current extension on the direct sum. The extension is a simple $C_{2}$-cofinite Möbius vertex algebra, and it is unique up to isomorphism. If $V$ is also a rational vertex operator algebra, then the extension is also rational, i.e., it is a simple regular vertex operator algebra.

Proof. We let $\mathcal{C}$ denote the subcategory of $V$-modules spanned by our group $A$ of units in $K_{0}(V)$. Then Proposition 3.1.21 implies we have a quasi-simple $\mathcal{C}$-current extension $W_{A}$ of $V$, together with a commutativity datum on simple modules $M_{A+i}$. By the evenness conjecture, the contragradient form on each $M_{A+i}$ is symmetric, so Corollary 3.2 .8 implies the commutativity datum is even, and we obtain a simple current extension of $V$. The remaining claims follow from Theorem 3.2.12.

\section{REFERENCES}

[SGA4]

[Bakalov-Kac 2006]

[Bakalov-Kirillov 2001]

[Borcherds 1986]

[Carnahan 2012]

[Creutzig-Kanade-Linshaw 2015]

[Dong-Griess-Höhn 1998]

[Dong-Lepowsky 1993]

[Dong-Li-Mason 1995]

[Dong-Lin 1994]

[Dong-Mason 2002]
M. Artin, A. Grothendieck, J.-L. Verdier, Théorie des topos et cohomologie étale des schémas III, Séminaire de Géométrie Algébrique du Bois-Marie 1962/64 Lecture Notes in Mathematics 305 Springer-Verlag, Heidelberg (1973).

B. Bakalov, V. Kac, Generalized vertex algebras "Lie theory and its applications in physics VI" Heron Press, Sofia, (2006) 3-25, https://arxiv.org/abs/ math/0602072

B. Bakalov, A. Kirillov, Jr., Lectures on tensor categories and modular functors University Lecture Series, Vol. 21, Amer. Math. Soc., Providence, RI, 2001.

R. Borcherds, Vertex algebras, Kac-Moody algebras, and the Monster Proc. Nat. Acad. Sci. USA, 83 no. 10 (1986) 3068-3071.

S. Carnahan, Generalized Moonshine IV: Monstrous Lie Algebras ArXiv preprint https://arxiv.org/abs/1208.6254

T. Creutzig, S. Kanade, A. Linshaw, Simple current extensions beyond semisimplicity Comm. in Contemp. Math., https://doi.org/10.1142/ S0219199719500019 ArXiv preprint https://arxiv.org/abs/1511.08754

C. Dong, R. Griess, G. Höhn, Framed vertex operator algebras, codes and the moonshine module Comm. Math. Physics 193 1998, 407-448. ArXiv preprint https://arxiv.org/abs/q-alg/9707008

C. Dong, J. Lepowsky, Generalized vertex algebras and relative vertex operators Progress in Mathematics 112 Birkhuser Boston, Inc., Boston, MA, (1993).

C. Dong, H. Li, G. Mason, Simple currents and extensions of vertex operator algebras Comm. Math. Phys. 180 no. 3 (1996) 671-707. ArXiv preprint https: //arxiv.org/abs/q-alg/9504008.

C. Dong, Z. Lin, Induced modules for vertex operator algebras Comm. Math. Phys. 179 (1996), 154-184. ArXiv preprint https://arxiv.org/abs/hep-th/ 9412038

C. Dong, G. Mason, Rational vertex operator algebras and the effective central charge Int. Math. Res. Not. 56 (2004) 2989-3008. ArXiv preprint https:// arxiv.org/abs/math/0201318 
[Eilenberg 1952]

[Eilenberg-Mac Lane 1953]

[Eilenberg-Mac Lane 1954]

[van Ekeren-Möller-Scheithauer 2015] J. van Ekeren, S. Möller, N. Scheithauer, Construction and Classification of Holomorphic Vertex Operator Algebras J. Reine Angew. Math. https: //doi.org/10.1515/crelle-2017-0046. ArXiv preprint https://arxiv.org/ abs/1507.08142

[Feingold-Frenkel-Ries 1991]

[Frenkel-Ben-Zvi 2004]

[Frenkel-Huang-Lepowsky 1993]

[Frenkel-Lepowsky-Meurman 1988]

[Frenkel-Zhu 1992]

[Fuchs-Gepner 1988]

[Fuchs-Runkel-Schweigert 2004]

[Gaberdiel-Neitzke 2000]

[Gepner-Witten 1986]

[Griess-Lam 2011]

[Guido-Longo 1995]

[Höhn 2002]

[Höhn 2003]

[Höhn-Scheithauer 2010]

[Huang 1994]

[Huang 1995]

[Huang 2001]

[Huang 2002]

S. Eilenberg, Homotopy groups and algebraic homology theories Proceedings of the International Congress of Mathematicians, Cambridge, Mass., 1950, vol. 2, Amer. Math. Soc., Providence, R.I., (1952) 350-353.

S. Eilenberg, S. Mac Lane, On the groups $H(\Pi, n)$, I Ann. Math. (2) 58 no. 1 (1953), 55-106.

S. Eilenberg, S. Mac Lane, On the groups $H(\Pi, n)$, II, Methods of computation Ann. Math. (2) 60 no. 1 (1954), 49-139.

A. Feingold, I. Frenkel, J. Ries, Spinor construction of vertex operator algebras, triality, and $E_{8}^{(1)}$ Contemporary Math., 121 AMS, (1991).

E. Frenkel, D. Ben-Zvi, Vertex algebras and Algebraic Curves Mathematical Surveys and Monographs $\mathbf{8 8}$ American Mathematical Society, Providence, RI, (2004).

I. Frenkel, Y. Huang, J. Lepowsky, On axiomatic approaches to vertex operator algebras and modules Mem. Amer. Math. Soc. 104 (1993) no. 494.

I. Frenkel, J. Lepowsky, A. Meurman, Vertex operator algebras and the Monster Pure and Applied Mathematics 134 Academic Press, Inc., Boston, MA, (1988). I. Frenkel, Y. Zhu, Vertex operator algebras associated to representations of affine and Virasoro algebras Duke Math. J. 66 no. 1 (1992) 123-168.

J. Fuchs and D. Gepner, On the connection between WZW and free field theories Nucl.Phys. B 294 (1988) 30-42.

J. Fuchs, I. Runkel, C. Schweigert, Vertex Operator TFT construction of RCFT correlators: III: simple currents Nuclear Physics B 694 (2004) 277-353. ArXiv preprint https://arxiv.org/abs/hep-th/0403157

M. Gaberdiel, A. Neitzke, Rationality, quasirationality and finite $W$-algebras Commun.Math.Phys. 238 (2003) 305-331. ArXiv preprint https://arxiv. org/abs/hep-th/0009235

D. Gepner and E. Witten String theory on group manifold Nucl. Phys. B 278 (1986) 493-549.

R. Griess, C. Lam, A new existence proof of the Monster by VOA theory Michigan Math. J. 61 Issue 3 (2012) 555-573. ArXiv preprint https: //arxiv.org/abs/1103.1414

D. Guido, R. Longo, The conformal spin and statistics theorem Commun. Math. Phys. 181 (1996) 11-35. ArXiv preprint https://arxiv.org/abs/ hep-th/9505059

G. Höhn, Genera of vertex operator algebras and three dimensional topological quantum field theories Vertex operator algebras in mathematics and physics (Toronto, ON 2000), 89-107, Fields Inst. Commun., 39 Amer. Math. Soc., Providence, RI, 2003. ArXiv preprint https://arxiv.org/abs/math/0209333 G. Höhn, Generalized moonshine for the baby monster Preprint (2003)

G. Höhn, N. Scheithauer, A generalized Kac-Moody algebra of rank 14 J. Algebra 404 (2014) 222-239. ArXiv preprint https://arxiv.org/abs/1009.5153 Y. Huang, A non-meromorphic extension of the moonshine module vertex operator algebra Contemporary Math., 193 AMS (1996), 123-148. ArXiv preprint https://arxiv.org/abs/hep-th/9406190

Y. Huang, A theory of tensor products for module categories for a vertex operator algebra, IV J. Pure Appl. Alg. 100 (1995) 173-216. ArXiv preprint https://arxiv.org/abs/q-alg/9505019

Y. Huang, Conformal-field-theoretic analogues of codes and lattices Kac-Moody Lie Algebras and Related Topics, Proc. Ramanujan International Symposium on Kac-Moody Lie algebras and applications, ed. N. Sthanumoorthy and K. C. Misra, Contemp. Math., Vol. 343 Amer. Math. Soc., Providence, RI, 2004, 131-145. https://arxiv.org/abs/math/0104150

Y. Huang, Differential equations and intertwining operators Comm. Contemp. Math. 7 (2005) 375-400. ArXiv preprint https://arxiv.org/abs/math/ 0206206 
[Huang-Kirillov-Lepowsky 2014]

[Huang-Lepowsky-Zhang 2007]

[Joyal-Street 1986]

[Kac 1997]

[Kawahigashi-Longo 2004]

[Lam 2001]

[Lam-Yamauchi 2006]

[Li 1997]

[MacLane 1952]

[Miyamoto 1996a]

[Miyamoto 1996b]

[Miyamoto 2010]

[Schellekens-Yankielowicz 1989]

[Schellekens 1992]

[Shimakura 2006]

[Stacks-Project]

[Yamauchi 2002]
Y. Huang, A. Kirillov, J. Lepowsky, Braided tensor categories and extensions of vertex operator algebras J. Commun. Math. Phys. 337 (2015) 11431159. https://doi.org/10.1007/s00220-015-2292-1 ArXiv preprint https: //arxiv.org/abs/1406.3420.

Y. Huang, J. Lepowsky, L. Zhang, Logarithmic tensor product theory for generalized modules for a conformal vertex algebra ArXiv preprint https: //arxiv.org/abs/0710.2687

A. Joyal, R. Street, Braided tensor categories Macquarie Mathematics report no. 860061, available at http://rutherglen.ics.mq.edu.au/ street/JS86. pdf A shorter version was published in Adv. Math. 102 (1993), no. 1, 20-78. V. Kac, Vertex Algebras for Beginners University Lecture Series 10, Amer. Math, Soc., Providence, RI (1997)

Y. Kawahigashi, R. Longo, Local conformal nets arising from framed vertex operator algebras Adv. Math. 206 2, 10 (2006) 729-751. ArXiv preprint http: //arxiv.org/abs/math/0407263

C. H. Lam, Induced modules for orbifold vertex operator algebras J. Math. Soc. Japan 53 No. 3 (2001) 541-557.

C. H. Lam, H. Yamauchi, On the structure of framed vertex operator algebras and their pointwise frame stabilizers Comm. Math. Phys. 277 no. 1 (2008) 237-285. ArXiv preprint http://arxiv.org/abs/math.QA/0605176

H. Li, Extension of Vertex Operator Algebras by a Self-Dual Simple Module J. Algebra 187 (1997) 236-267.

S. Mac Lane, Cohomology theory of abelian groups Proceedings of the International Congress of Mathematicians, Cambridge, Mass., 1950, vol. 2, Amer. Math. Soc., Providence, R.I., (1952) 8-14.

M.Miyamoto, Binary codes and vertex operator (super) algebras J. Algebra 181 (1996) 207-222.

M. Miyamoto, Representation theory of code vertex operator algebra J. Algebra 201 (1998) 115-150. ArXiv preprint https://arxiv.org/abs/hep-th/ 9612032

M. Miyamoto, $A \mathbb{Z}_{3}$-orbifold theory of lattice vertex algebra and $\mathbb{Z}_{3}$-orbifold constructions Symmetries, Integrable Systems and Representations, Springer Proceedings in Mathematics \& Statistics 40 (2013) 319-344. ArXiv preprint https://arxiv.org/abs/1003.0237

A. N. Schellekens and S. Yankielowicz, Extended chiral algebras and modular invariant partition functions Nucl. Phys. 327 (1989), 673-703.

A. N. Schellekens, On the classification of meromorphic $c=24$ conformal field theories Teoret. Mat. Fiz., 95:2 (1993) 348-360. ArXiv preprint http: //arxiv.org/abs/hep-th/9205072

H. Shimakura, Lifts of automorphisms of vertex operator algebras in simple current extensions Math. Z. 256 (2007), 491-508. ArXiv preprint http:// arxiv.org/abs/math/0610671

The Stacks Project Authors, Stacks Project http://math.columbia.edu/ algebraic_geometry/stacks-git/

H. Yamauchi, Module categories of simple current extensions of vertex operator algebras J. Pure Appl. Algebra 189 (2004) 315-328. ArXiv preprint http: //arxiv.org/abs/math/0211255 